\title{
Characterization of foetal hepatic cells during rat liver development
}

\author{
Dissertation \\ zu Erlangung des Doktorgrades \\ der Mathematisch-Naturwissenschaftlichen Fakultäten \\ der Georg-August-Universität zu Göttingen
}

vorgelegt von

Abderrahim Elmaouhoub

Aus Rabat, Marokko

Göttingen 2006 
D7

Referent:

Prof. Dr. R. Hardeland

Korreferent:

Prof. Dr. E. Wimmer

Tag der mündlichen Prüfung: 05.07.2006 
Table of contents...............................................................................

Table of figures............................................................................

Abbreviations............................................................................... VIII

Abstract..................................................................

Table of contents

1.INTRODUCTION..............................................................................

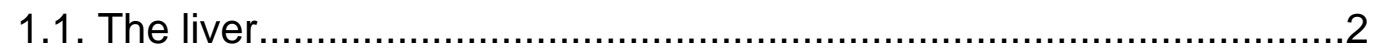

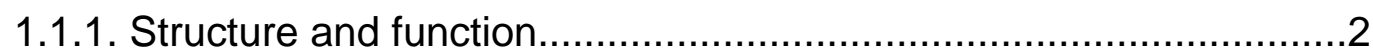

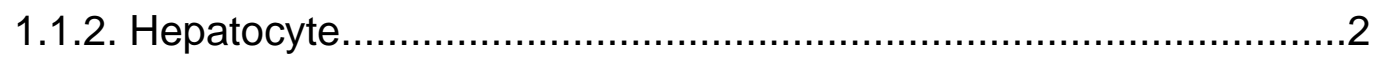

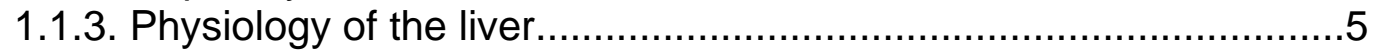

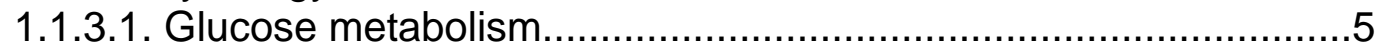

1.1.3.2. Lipide/Lipoprotein and cholesterol........................................5

1.1.3.3. Amino Acid, protein synthesis and ammonia metabolism............6

1.2. Embryology of the rat and liver development.................................6

1.3. Embryonic/fetal liver and hematopoeisis...................................... 8

1.4. Specific Markers for hepatic and intrahepatic bile duct phenotype.....9

1.4.1. Albumin and alpha-fetoprotein ............................................... 9

1.4.2. Prospero-related homeobox transcription factor-1 (Prox1).............10

1.4.3. Cytokeratins-7 and -19 (CK-7 and CK-19)...............................11

1.5. Background..................................................................11

1.5.1. Kinetics for albumin and AFP mRNA expression during liver development ..................................................................12

1.5.2. Distribution of albumin and AFP producing cells during liver development....................................................13

1.5.3. Synthesis and secretion of albumin and AFP............................13

1.5.4. Cytokeratins-7 and -19 expression during intrahepatic bile duct development.......................................14

1.5.6. Cytokines regulating hematopoiesis in embryonic and adult state. 15

1.6. Objective of the work........................................................16

2. MATERIALS AND METHODS.......................................................18

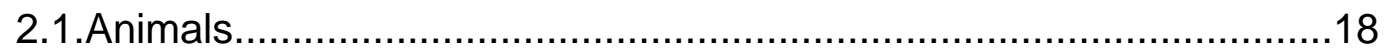

2.2. Chemicals, Enzymes and Kits................................................

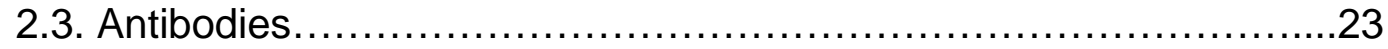

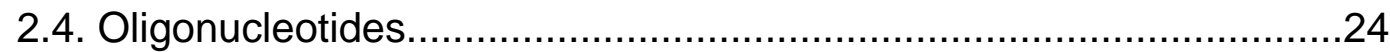

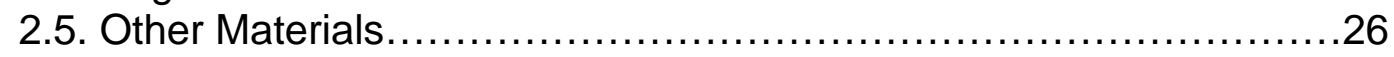




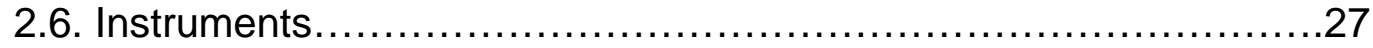

2.7. Embryos, hepatoblasts isolation and culture...............................30

2.7.1. Dissection of the embryos........................................ 30

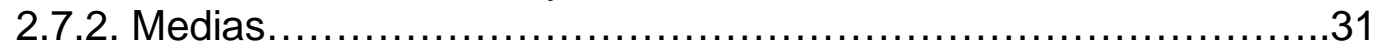

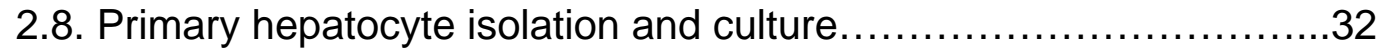

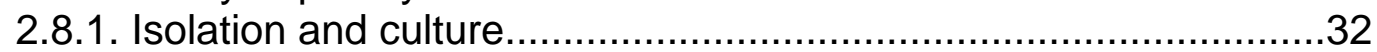

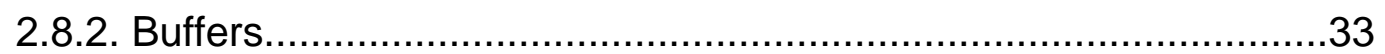

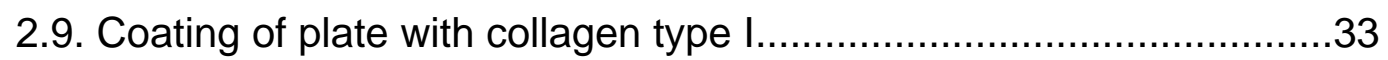

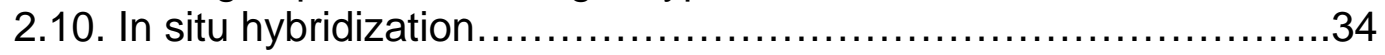

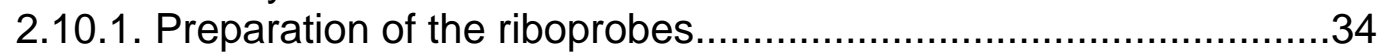

2.10.2. First-Strand cDNA Synthesis using M-MLV Reverse

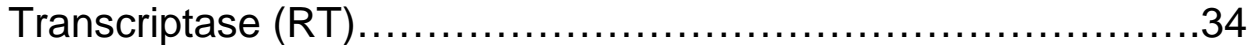

2.10.3. Amplification of DNA by polymerase chain reaction (PCR)..........35

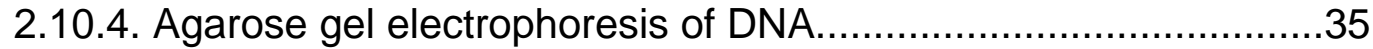

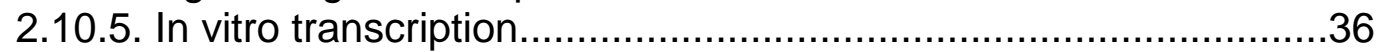

2.10.6. Whole-mount in situ hybridization with digoxygenin-labeled

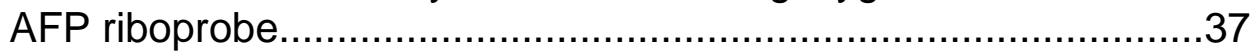

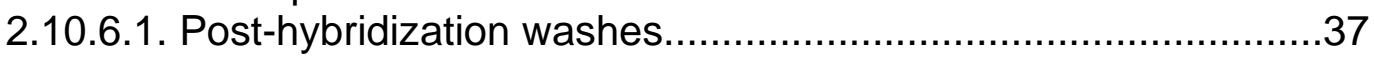

2.10.6.2. Blocking of embryos and antibody binding...........................38

2.10.6.3. Post-antibody washes and signal detection...........................38

2.10.7. Single and double in situ hybridization...................................38

2.10.7.1. Post-hybridization washes and signal detection......................39

2.10.7.2. Deactivation of the first alcaline phosphatase enzyme.............40

2.10.8. In situ hybridization in explanted hepatoblasts.........................40

2.10.9. Solutions and buffers ...........................................40

2.11. Proliferation assay in developing liver by PCNA immunostaining...44

2.11.1. PCNA-staining by immunoperoxidase technique........................44

2.11.2. Buffers................................................................................. 45

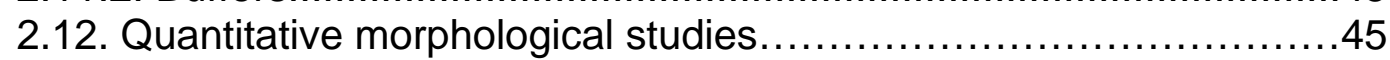

2.13. RNA extraction.................................................................45

2.13.1. Isolation of RNA by density-gradient ultracentrifugation..............45

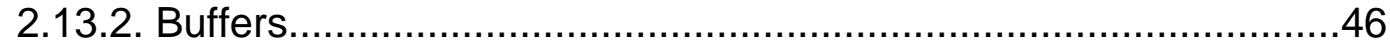

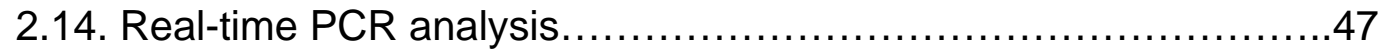

2.15. Fluorescence immunostaining .............................................48

2.16. Quantitative analysis of Prox1-, CK19- and AFP-specific immunohistochemical reactions in embryonal and foetal rat livers .............................49

2.17. Radioactive biosynthetic labeling, immunoprecipitation and SDS-PAGE Analysis.............................................50

2.17.1. Radioactive biosynthetic labeling with ${ }^{35}$-S Methionine...............50

2.17.2. Preparation of cell lysates and measurement of total labeled proteins after trichloracetic acid precipitation............................50

2.17.3. Immunoprecipitation of albumin and AFP by polyclonal Anti-albumin and anti-AFP antibodies...................................51

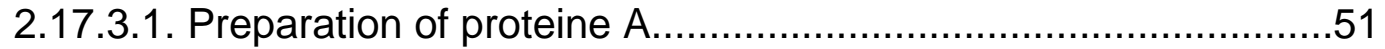

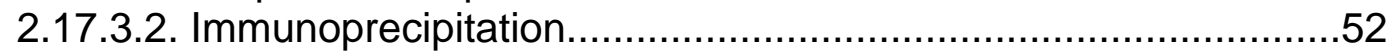

2.17.3.3. SDS-polyacylamid gel elctrophoresis (SDS-PAGE) analysis.....52 


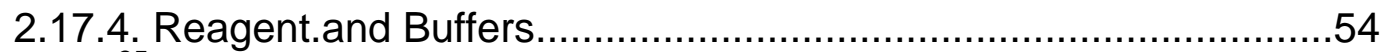

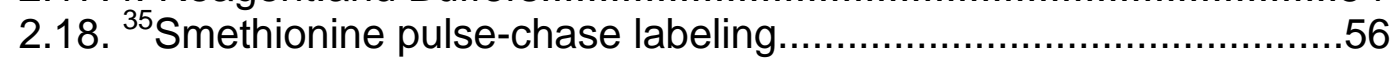

2.19. Interleukin-6 (IL6) treatment.....................................................5

2.20. Analysis of albumin and AFP gene expression in hepatoblasts

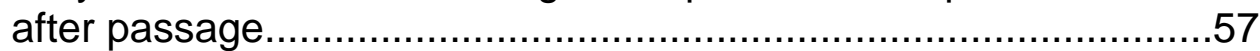

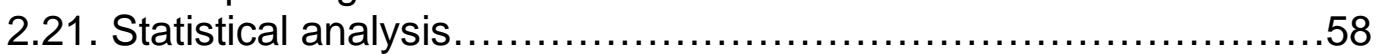

3. RESULTS

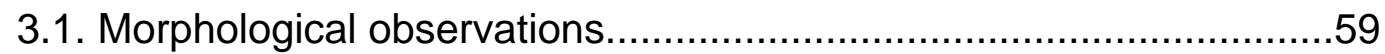

3.2. Albumin and AFP gene expression at the time

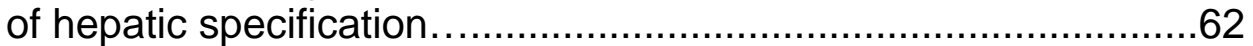

3.2.1. Identification of albumin and AFP mRNA expression in ventral foregut.

3.2.2. Synthesis and secretion of albumin and AFP by endodermal cells derived from ventral foregut.

3.3. Characterization of endodermal cells generated from ventral foregut.

3.4. Cellular analysis of albumin and AFP expressing cells during liver development.

3.5. Quantitative analysis of albumin and AFP mRNA expression during liver development.

3.6. Kinetics for synthesis and secretion of albumin and AFP during liver development.

3.7. Expression of prox1 and cytokeratin-7 and -19 during liver development.

3.8. Effect of interleukin-6 on synthesis and secretion of albumin and AFP.

3.9. Characterisation of hepatoblats after different passage.

3.10. Changes in gene expression of hematopoietic regulatory cytokines during liver development.

4.1. Albumin and AFP gene expression at the time of hepatic

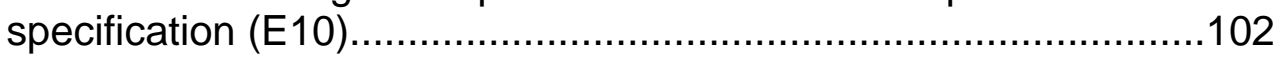

4.2. Characterisation of endodermal cells.......................................103

4.3. Cellular analysis during liver development................................. 105

4.4. albumin and AFP mRNA expression in developing liver..............106

4.5. Synthesis and secretion of albumin and AFP during liver development.

4.6. Characterization of intrahepatic bile duct phenotype during liver development. 
4.7. Effect of interleukin 6 (IL6) on albumin and AFP gene expression in developing liver 110

4.8. Identification of hepatic phenotype in passaged hepatoblasts.......111

4.9. Regulation of hematopoiesis during liver development.............112

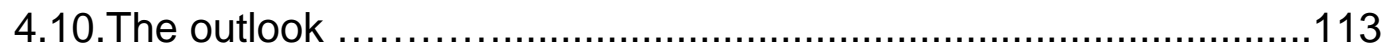

5. ZUSAMMENFASSUNG.................................................................115

6. REFERENCE LIST...................................................................117 ACKNOWLEDGMENTS 
Tables of figures

Figure 1: Three dimensional presentation display vascular supply and sinusoidal structure of the liver.

Figure 2: Structure of hepatocyte

Figure 3: Embryonic development of the rat 7

Figure 4: Morphological and cultural features of endodermal cells derived from ventral foregut region

Figure.5: Phase-contrast microscopical pictures of primary rat hepatoblasts isolated at developmental stages E12 (12), E14 (14), E16 (16), E18 (18), E20 (20) and primary hepatocytes generated from new born (NB) and adult liver (Ad).....

Figure 6: Expression of albumin and alpha-fetoprotein (AFP) mRNA in embryos(A) and explanted endodermal cells(B) detected by whole mount and in vitro in situ hybridization respectively. .63

Figure 7: A) Synthesis and secretion of Albumin (a) and alpha-fetoprotein (AFP) (b) by endodermal cells generated from venral foregut at E10.

Figure 8: Real-time-PCR analysis of mRNA extracted from cultured endodermal cells, hepatoblasts and hepatocyte. Albumin (A) and AFP (B) mRNA were expressed in cultured endodermal cells derived from ventral foregut.... .66

Figure 9: Immunofluorescence staining of explanted emdodermal cells (E10) and hepatoblasts from embryos at E12. DAPI and anti HNF4aplha staining shows that in explanted endodermal cells generated from ventral foregut some cells are HNF4alpha positive. Hepatoblasts from E12 are used as positive control, they are HNF4alpha positive.

Figure 10: Real time PCR analysis of HNF4alpha, Beta-catenin, Prox1, foxa2, GATA-4 and BMP4 mRNA expression in explanted endodermal cells (E10) derived from ventral foregut and hepatoblasts derived from embryos at 12 days of gestation (E12). Ribosomal 18S was used as housekeeping gene. 68

Figure 11: Albumin mRNA-expression during liver development assessed

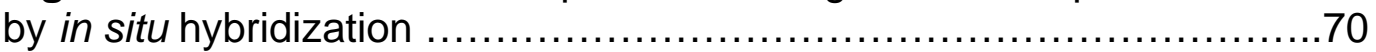


Figure 12: AFP mRNA-expression during liver development assessed by in situ hybridization .71

Figure 13: The ratio of albumin and AFP producing cells to total cells during liver development................................................

Figure 14: Proliferation estimated by the number of positive cells expressing proliferating cell nuclear antigen (PCNA) during liver development . .73

Figure 15: The ratio of albumin and AFP expressing cells to proliferating cells during liver development.. 74

Figure 16: Co-localisation of albumin and AFP mRNA during liver development...........................................................

Figure 17: Albumin and AFP mRNA expression in cultured hepatoblasts during liver development. .76

Figure 18: Quantitative analysis of albumin (A) and AFP (B) mRNA expression in whole liver (in vivo) during liver development using realtime-PCR experiment 78

Figure 19: Quantitative analysis of albumin (A) and AFP (B) mRNA expression in cultured hepatoblasts (in vitro) during liver development using real-time-PCR experiment .79

Figure 20: Synthesis and secretion of Albumin and alpha-fetoprotein (AFP) in viable hepatoblasts at developmental stage E12, E14, E16, E18, E20 and in viable hepatocyte at new born and adult state. 81

Figure 21: Kinetics for albumin and AFP in hepatoblasts during liver development measured by pulse chase experiment .82

Figure 22: Kinetics for albumin in hepatoblasts after 2 and 9 DIV (DIV: days in vitro) at E12, E14, E16 and E18 measured by pulse chase experiment. 86

Figure 23: Prox1-, CK-19-, and CK-7-specifc immunohistochemical reactions in embryonic rat liver at E14 and E16. .88

Figure 24: Immunohistochemical reactions with anti-Prox1 (red), antiAFP (red and green) and anti-CK-19 (green) antibodies. counterstaining of nuclei with DAPI (blue).. .89 
Figure 25: Reactions with anti-Prox1 (red) anti-CK-19 (green), anti-CK-7 (green), anti-HepPar-1 (green) and anti-connexin 32 (green) antibodies, counterstaining of nuclei with DAPI (blue) in the liver of rat embryos at 18 days of gestation. 90

Figure 26: Prox1-, CK-19-, AFP-specific immunohistochemical reactions in embryonic rat liver at E18.

Figure 27: Prox1-, CK-19-specific immunocytochemical reactions in cultured hepatoblasts generated from developmental stages E12, E14 and E18.

Figure 28: Reactions with anti-Prox1 (red) anti-CK19 (green), anti-CK7 (green), anti-HepPar-1 (green) and anti-connexin 32 (green) antibodies, counterstaining of nuclei with DAPI (blue) in adult rat liver.

Figure 29: (A) Effect of interleukin-6 (IL-6) on synthesis and secretion of albumin and AFP in hepatoblasts at developmental stage E12, E14, E18 (B) Real time PCR analysis of interleukin-6 receptor mRNA-expression in unstimulated hepatoblasts. The expression was normalized with the endogenous control of GAPDH.

Figure 30: Synthesis and secretion of Albumin and AFP in passaged hepatoblasts derived from 12 and 14 days of gestation.... 96

Figure 31: Real-time PCR analysis of albumin (A) and AFP (B) mRNAexpression after passage of hepatoblasts. The expression was normalized with the endogenous control of GAPDH.

Figure 32: Real-time-PCR -analysis of mRNA from whole liver (in vivo) and cultured hepatic cells (in vitro). Livers were obtained and the hepatic were cultured at different developmental stage as described in materials and methods. Fold change of granulocyte-macrophage colony-stimulating factor (GM-CSF) (A), granulocyte colony-stimulating factor (G-CSF) (B), stem cell factor (SCF) (C) and Erythropoietin (Epo) (D) gene-expression during liver development.............................................. 101

Figure 33: represents the possible changes in characteristics of liver cells during liver development. 


\section{Abbreviations}

$\begin{array}{ll}\text { AFP } & \text { Alpha-fetoprotein } \\ \text { ANOVA } & \text { Analysis of variance } \\ \text { APS } & \text { Ammonium persulfate } \\ \text { BEC } & \text { Biliary epithelial cells } \\ \text { BLDs } & \text { Bioartificial liver devices } \\ \text { BMPs } & \text { Bone morphogenetic proteins }, \\ \text { CoH } & \text { Canals of hering } \\ \text { CHAPS } & \text { 3-[(3-Cholamidopropyl)dimethylammonio]-1- } \\ & \text { propanesulfonate } \\ \text { CK7 and CK19 } & \text { Cytokeratins 7 and 19 } \\ \text { DAB } & \text { 3,3-Diaminobenzodine } \\ \text { DAPI } & \text { 4'-6-Diamidino-2-phenylindole } \\ \text { DIV } & \text { Days in vitro } \\ \text { DEPC } & \text { Diethylpyrocarbonat } \\ \text { DOC } & \text { Deoxycholic acid } \\ \text { DMF } & \text { N-N-Dimethylformamid } \\ \text { EDTA } & \text { Ethylenediaminetetraacetic } \\ \text { Epo } & \text { Erythropoietin } \\ \text { E } & \text { Days of gestation } \\ \text { ER } & \text { Endocytoplasmic reticulum } \\ \text { FCS } & \text { Fetal calf serum } \\ \text { Foxa2 } & \text { Forkhead box proetins A2 } \\ \text { FGFs } & \text { Fibroblast growth factors } \\ \text { GER } & \text { Glatt endoplasmic reticulum } \\ \text { GATA4 } & \text { GATA binding protein 4 } \\ \text { GM-CSF } & \text { Granulocyte-macrophage colony-stimulating factor } \\ \text { G-CSF } & \text { Granulocyte colony-stimulating factor } \\ \text { IL6 } & \text { Interleukin-6 } \\ \text { IL6 rec } & \text { Interleukin-6 receptor } \\ \text { LM+SDS } & \text { Lysis Mix with Sodium n-Dodecyl Sulfate } \\ \text { LM-SDS } & \text { Lysis Mix without Sodium n-Dodecyl Sulfate } \\ \text { LDL } & \text { Low density lipoprotein } \\ \text { MEM } & \text { Minimal essential medium } \\ \text { Na-As-Bi-P } & \text { Naohtol-AS-Bl-Phosphat } \\ \text { OSM } & \text { Oncostatin M } \\ \text { PCNA } & \text { Proliferating cell nuclear antigen } \\ \text { PFA } & \text { Paraldehyde } \\ & \end{array}$


Prox1 RT-PCR PMSF

RER

SCF

TNF-R1

TEMED

TCA
Prospero-Related Homeobox- transcription Factor-1 Reverse trancriptase-polymerase chain reaction Phenylmethylsulfonylfluoride

Rough endoplasmic reticulum Stem cell factor

Tumor Necrosis Factors- receptor 1 $\mathrm{N}, \mathrm{N}, \mathrm{N}^{\prime}, \mathrm{N}^{\prime}$-Tetramethylethylenediamine Trichloracetic acid 


\section{ABSTRACT}

During embryonic development the embryonic/foetal liver is the site where hepatogenesis and hematopoiesis take place. Hepatoblasts have been partly characterized in several previous studies. However, their characterization in the developing liver has not been previously studied. Therefore, the purpose of the current work was to characterize, in a rat model, hepatoblasts in vitro and in vivo during liver development. We consider the progress from early developmental stage, 10 days of gestation (E10) when the liver first starts to develop, to adulthood.

Albumin and alpha-fetoprotein (AFP) are the main hepatic markers and are the earliest synthetic products of hepatoblasts during liver development. In this study, we established a reliable method for the first time using a sensitive radioactive biosynthetic labelling, to analyse the albumin and AFP synthesis and secretion capacity of endodermal cells derived from ventral foregut region (E10). It seems that the whole program controlling the regulation of gene expression, synthesis and secretion of albumin and AFP already acts at the earliest developmental stage, when specification of hepatic endoderm appears. The present study shows that explanted endodermal cells from ventral endoderm can express HNF4alpha, Prox1, beta-catenin, BMP-4, Foxa2, and GATA-4.

In the second part of this work we were interested in the development of the liver after it was clearly identifiable as a separate organ (from E12 to adulthood). We demonstrated that during the embryonic and foetal stages about $50 \%$ of liver cells are engaged in both albumin and AFP gene expression. In addition the ratio of albumin and AFP producing cells to proliferating cells increases during embryonic stage. At 18 days of gestation the ratio of albumin and AFP producing cells to proliferating cells reaches its maximum. 
Quantitatively we found that at 18 days of gestation, albumin and alphafetoprotein mRNA reaches a maximum and a high rate of synthesis and secretion of albumin and AFP was observed. Additionally, it was observed that at the embryonic stage (from E12 up to E16) albumin and alphafetoprotein were synthesized and secreted at different rates. From 18 days of gestation to birth the kinetics of synthesis and secretion of albumin is similar to the kinetics in mature hepatocytes.

In the rat embryonic (E12 and E14) and foetal (E18) liver three cell populations were identified. Two of these had a unipotent character, developing into either hepatic lineage (Prox1 positive cells/CK-19 negative cells) or into intrahepatic bile duct lineage (Prox1 negative/CK19 positive cells). The third population retained its bipotent character (CK19 and prox1 positive cells), being able to differentiate into hepatic or bile duct epithelial cells. At this stage CK- 7 was also first detected.

Lastly hematopoiesis in the embryonic liver was investigated. A high expression of genes coding for factors which regulate hematopoiesis, such as GM-SCF, G-CSF, SCF and Epo, was observed at 12 and 14 days of gestation. 


\section{INTRODUCTION}

Liver transplants are currently the only available and effective treatment for patients with end-stage liver failure. There is, however, an increasing shortage of donor livers for clinical therapies. Improved cell therapies may serve as an alternative approach for the treatment of these patients, and could offer a ray of hope for many suffering from liver diseases. Recent studies have proposed different types of stem/progenitor cells for transplantation. Suggestions include: Embryonic stem cells (Kania et al., 2003); hematopoietic stem cells (HSCs) (Avital et al., 2002; Alison et al., 2004; Kang et al., 2005); mesenchymal stem cells (Avital et al., 2002; Alison et al., 2004; Kang et al., 2005; Hong et al., 2005; Teramoto et al., 2005); ovale cells, also referred to as intrahepatic adult stem cells (Lazaro et al., 1998; He et al., 2004; Matsusaka et al., 2000; Yoon et al., 2004); and hepatoblasts (Mahieu-Caputo et al., 2004; Rogler, 1997; Stamp et al., 2005). Furthermore bioartificial liver devices (BLDs), gene therapy and administration of exogenous factors to stimulate normal physiological responses have been discussed.

Hepatoblasts might also be a candidate for therapeutical use in liver failures. They have been partially characterized in several previous studies (Sigal et al., 1994; Tanimizu et al., 2004; Rogler, 1997; Stamp et al., 2005), but their characterization in the developing liver remains unclear. In the current study we focussed our attention on the characterization of hepatoblasts in vivo and in vitro in the rat, from early developmental stage (10 days of gestation, the time when the liver starts to develop) to adulthood. 


\subsection{The liver}

\subsubsection{Structure and function}

The liver is a large parenchymal organ consisting of several separate lobes. Its weight $(1.5-1.8 \mathrm{~kg})$ represents about $2 \%$ of the total body weight in the human. The liver is the central organ of nutrient digestion and processing, where most of the individual metabolism occurs. Furthermore it is responsible for the synthesis of the serum proteins which regulate the oncotic pressure and the retention of water within the vessels. The liver is the only organ with two separate afferent blood supplies (Fig. 1). The hepatic artery provides oxygenated blood and the portal vein brings in venous blood rich in nutrients and hormones from intestine and pancreas (Desmet V.J. et al., 1994). The main cell types resident in the liver are hepatocytes, bile duct epithelium, stellate cells (Ito cells), kupffer cells, vascular endothelium, fibroblasts, and leukocytes (Ramadori and Saile, 2002).

\subsubsection{Hepatocytes}

The hepatocytes are one of the largest cells populations of the body. Individually they have a size of $20-30 \mu \mathrm{m}$ with a volume of $11,000 \mu^{3}$ (estimations vary between 10,000 and $60,000 \mu^{3}$ ). Their size however can vary considerably depending on age, location, the blood flow and metabolic load. A hepatocyte is polyhedric and possesses 5-12 facettes. Of these, one to three is in contact with sinusoidal blood, whereas four to nine are in contact with the biliary poles of the neighboring cells. 


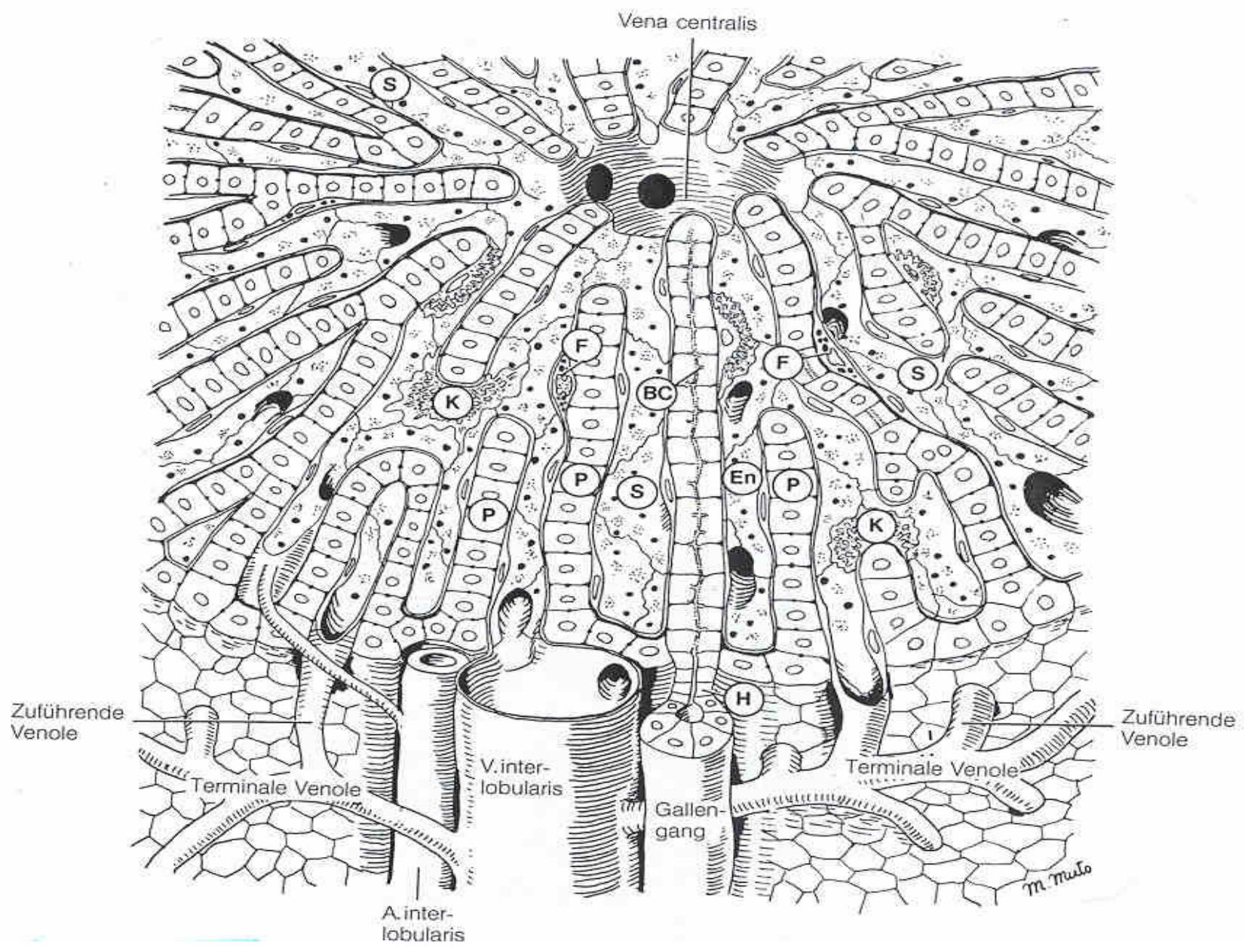

Figure 1: Three dimensional presentation display vascular supply and sinusoidal structure of the liver. Blood from Portal vein (V.interlobularis) and hepatic artery (A. interlobularis) enters the hepatic sinusoids one or two cells from the edge of the lobule, mixes in the sinussoids(S), delivers oxygen and nutrients to the liver cells, picks up carbon dioxide and metabolic products from the liver cells, and drains into the central vein (Vena centralis). Liver plate $(P)$ is a single layer of hepatocyte. Endothelial (En) cells form walls of the sinusoids and make openings between sinusoids and hepatocytes. Kupffer cells $(\mathrm{K})$ are located in the sinusoids and Ito cells (F) are located in spaces between endothelial cells and hepatocytes. Bile canaliculi (BC) drain bile into interlobular bile ducts in a direction opposite from the blood flow. H: canal of Hering (Grompe M. and Finegold M. J., 2001). 
The hepatocyte represents the main cell type in the liver (about $90 \%$ of the weight of the liver). It is one of the most metabolically active cell types of the body. Their ability to manage several complex functions at the same time is due to the fact that they contain a large number of organelles. The most abundant are the endoplasmic reticulum (ER), mitochondria, lysosomes and peroxysomes (Fig. 2).

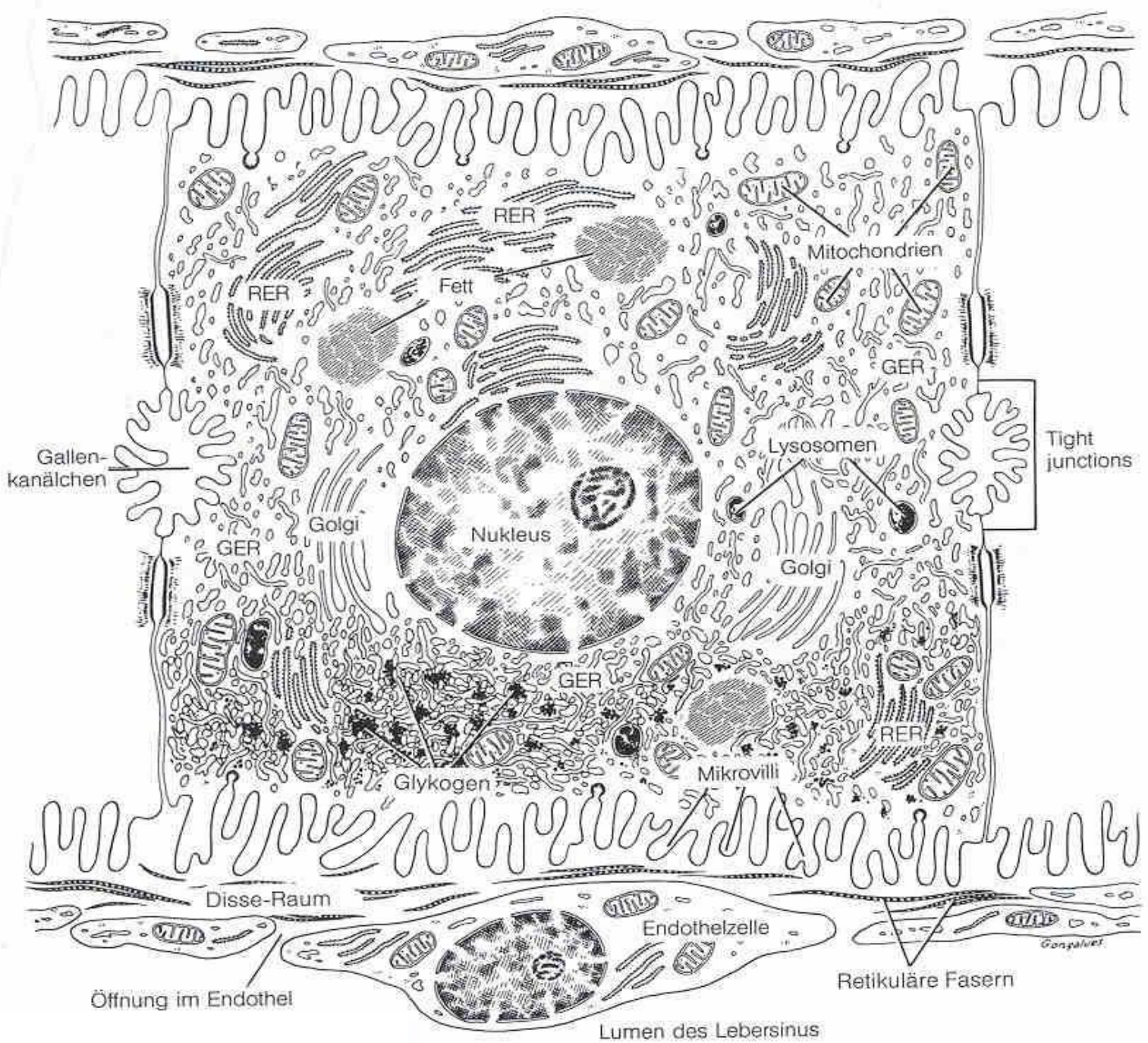

Figure 2: Structure of hepatocyte. RER: rough endoplasmic reticulum. GER: glatt endoplasmic reticulum. (Junqueira L. C. and Carneiro J., Histologie, 1996). 


\subsubsection{Physiology of the liver}

The liver has many functions that involving storage, metabolism, production and secretion. Furthermore, the hepatocytes are involved in the synthesis of bile acids, bile formation, and the processing of absorbed nutrients and xenobiotics. They are also responsible for maintenance of glucose, amino acid, ammonia and bicarbonate homeostasis in the body. They participate in the synthesis of most plasma proteins, and the storage and processing of signal molecules.

\subsubsection{Glucose metabolism}

The liver plays a pivotal role in glucose metabolism of the organism. It regulates the blood glucose level by glycogenolysis or gluconeogenesis in case of need and glycogen synthesis or glycolysis and lipogenesis when it is present in excess. Several factors are responsible for controlling the reversible switch between glycogenolysis/gluconeogenesis and glycogen synthesis/ glycolysis, such as substrate concentrations, hormone levels, hepatic nerves, the hepatocellular hydration and zonal hepatocyte heterogeneity (Jungermann and Thurman, 1992; Kaiser, 1998). Glycogen synthesis and glycolysis are predominantly regulated by the portal glucose concentration, with insulin and parasympathic nerves being auxiliary factors. Glycogenolysis and gluconeogenesis on the other hand, are initiated by glucagon and sympathic nerves but inhibited by high portal glucose concentration.

\subsubsection{Lipid/Lipoprotein and cholesterol}

The liver plays a central role in synthesis and metabolism of fatty acids, lipids and phospholipids, which are delivered into the blood as plasmalipoprotein. The liver has also the ability to synthesize cholesterol. 
The hepatocyte possess the low density lipoprotein (LDL) receptor, which is capable of cholesterol uptake. Excess of cholesterol is the basis for bile acid synthesis. It is directly shifted from the cell and secreted into the bile.

\subsubsection{Amino acid, protein synthesis and ammonia metabolism}

The liver participates in the amino acid homeostasis in the body. Excess amount of amino acids leads to their breakdown and utilization for protein synthesis as well as glycogen synthesis and simultaneously, inhibits amino acid generation by proteolysis. The liver continuously produces and secretes proteins including most of the plasma proteins. The major secreted protein is albumin (50\% of the secreted proteins)(Quinlan et al., 2005), which is secreted rapidly and is difficult to find it intracellularly. The portal blood contains high concentration of ammonia, which is derived from the intestinal mucosa, glutamine and intestinal microorganisms. However, ammonia is also produced by the hepatocytes during the processing of amino acids. The detoxification of ammonia occurs by both liver-specific urea synthesis and glutamine synthesis.

\subsection{Embryology of the rat and liver development}

The embryonic development of the rat is similar to the vertebrates where the sperm cell penetrates into the ova (fertilization). After that, the chromosomes of the two germ cells merge forming the metaphase plate of the first cleavage spindle. After several cleavages the blastocyte stage is reached between 80 and 110 hours after mating. On the fifth day of gestation begins the implantation stage, in which the blastocyte loses the zona pellucida. During the sixth and seventh gestation days, the blastocyte approaches the uterine surface, increase in size and then adheres to the uterine epithelium in several places (Hebel R. and Melvin W., 1986). 
The gastrulation begins on the eigth day of gestation (E8). It starts with the formation of the yolk sac and the three germ layers ecto, meso and endoderm. The formation of somites begins at the end of E9 and during E10. In 2 somites embryos, the foregut is a cone shaped elevation of the endoderm and the primordial germ cells are recognizable within the hindgut epithelium. At 10 day of gestation (4 somites stage), the foregut and hindgut become visible and the process of liver development starts. It begins with a connection of the foregut endoderm with the developing heart. Bone morphogenetic proteins (BMPs) and fibroblast growth factors (FGFs) signals arise from the septum transversum and the developing heart respectively, and transcription factors such as GATA, Foxa1 and Foxa2 (Zaret, 1996); Zhao et al., 2005; Serls et al., 2005; Deutsch et al., 2001) activate liver specific genes which regulate the hepatogenesis. On day 12 of gestation the liver is developed (Fig. 3).

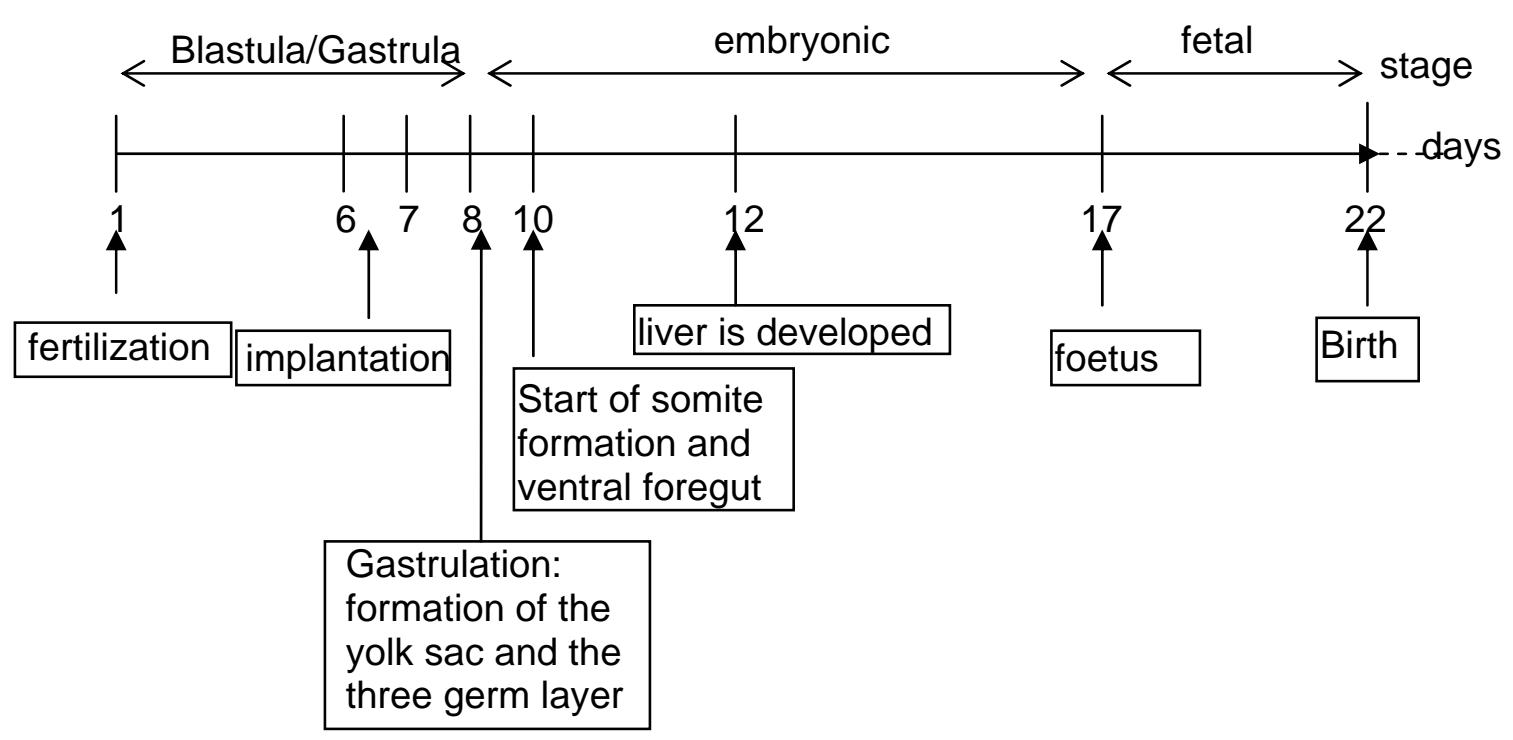

Figure 3: Embryonic development of the rat (Hebel R. and Melvin W., 1986). 


\subsection{Embryonic/foetal liver and hematopoiesis}

The embryonic liver represents the major site of hematopoiesis during embryonic development (Chang et al., 2005; Fukuda, 1974; Chang et al., 2005)). Along with maturation of the bone marrow and spleen, hematopoietic cells relocate from the liver to their final destinations, while the liver starts organizing its own structure and develops numerous metabolic functions toward adult.

During primitive (or embryonic) erythropoiesis (from E9 to E13 in the rat), which takes place first in yolk sac and then continue in embryonic liver, erythrocytes are distinguishable from those in definitive (or adult) erythropoiesis (from 13 to 20 in the rat) by their morphology. Primitive erythrocytes are nucleated cells containing embryonic as well as adult hemoglobin, whereas definitive erythrocytes are small nonnucleated red cells committed only to adult hemoglobin synthesis (Lin et al., 1996).

In yolk sac and early hepatic hematopoiesis, primitive macrophages develop from hematopoietic stem cells. They enter the blood stream and migrate into the embryonic liver before the initiation of hepatic hematopoiesis. They become mature and then transform into Kupffer cells. Myelopoiesis and monocytopoiesis are not active in yolk sac. Monocytic cells develop during hepatic hematopoiesis after the development of primitive macrophages, then move into the bone marrow in late ontogeny, forming a monocyte-derived macrophage population in tissues (Naito et al., 1990; Takahashi et al., 1996). 


\subsection{Specific markers for hepatic and intrahepatic bile duct phenotype}

\subsubsection{Albumin and alpha-fetoprotein (AFP)}

Albumin and alpha-fetoprotein (AFP) are two secretory plasma proteins that represents the members of albuminoid gene family consisting of four members to date: albumin, vitamin D-binding (Gc) protein, alphafetoprotein and alpha-albumin (McLeod and Cooke, 1989; Lichenstein et al., 1994). The genes encoding albumin and AFP are located in the same chromosome, positioned close to each other and have a common direction of transcription, despite their different regulation process at the transcription level (Belanger et al., 1994; Sargent et al., 1981; Jagodzinski et al., 1981). Albumin and alpha-fetoprotein are highly homologous in primary structure. They consist of three homologous domains and perform similar functions like maintenance of oncotic pressure, binding and delivering of endogenous and exogenous compounds including fatty acids, metal ions, hormones and drugs to different tissues.

Albumin is a $66 \mathrm{kD}$, single chain polypeptide that is produced in liver and forms a large proportion of all plasma proteins. It is also produced by others organs such as mammary gland, tongue, intestine, lymph gland, testicle and uterus, but in less amount than the liver (Shamay et al., 2005).

Compared to albumin, alpha-fetoprotein is a single chain glycoprotein that is normally produced in the foetus during its development but its gene expression is repressed reversibly in adult liver. It can be expressed by oval cells during the course of liver regeneration induced by partial hepatectomy, when up to $2 / 3$ of the organ is removed surgically or by acute $\mathrm{CCl}_{4}$ intoxication that causes necrosis of the hepatocytes bordering central veins (Tournier et al., 1988), (Bisgaard et al., 1994). It can be used in adults as a tumour marker in the case of primary hepatic tumors. The 
main product of AFP gene transcription in foetal liver is a $2.1 \mathrm{~kb}$ mRNA (corresponding to polypeptide chain of $68 \mathrm{kD}$ and $70 \mathrm{kD}$ ) (Lemire and Fausto, 1991; Lazarevich, 2000). Other sequence of AFP mRNA (1.7kb, $1.4 \mathrm{~kb}$ and $1 \mathrm{~kb}$ ) were detected in foetal, regenerating liver and in carcinogenesis (Petropoulos et al., 1985), (Wan and Chou, 1989).

\subsubsection{Prospero-related homeobox transcription factor-1 (Prox1)}

Porspero-related homeobox 1 (Prox1) is a divergent transcription factor with two highly conserved domains, a homeobox and a prospero domain. It was first detected in the hepatic endoderm of the mouse at the 7 to 8 somites stage (E8.5) (Sosa-Pineda et al., 2000). In the endoderm, Prox1 expression is confined to a short segment that gives rise to liver and pancreas, where it remains expressed into adulthood (Burke and Oliver 2002; Dudas et al. 2004). Prox1 plays no role in hepatic specification. It is required for the migration of hepatocytes into the septum transversum. In Prox1 deficient mice, the hepatoblasts fail to migrate into the neighbouring mesenchyme. They are abnormally clustered near the hepatic diverticulum, and remain invested by a continuous basal lamina. Prox1 null mice die around E14.5, and show a 70\% reduction of the liver size (Sosa-Pineda et al. 2000).

Prox1 is an early marker of hepatoblasts in mouse and rat embryos (Dudas et al. 2004). In both mice and rats Prox1 expression in hepatocytes persists into adulthood. It is not expressed in bile duct epithelial cells, which are positive for cytokeratin-7 (CK-7) (Dudas et al. 2004), nor is it expressed in liver endothelial cells, which are positive for CD31. The expression pattern of Prox1 is highly conserved in vertebrates and the human (Dudas et al. 2004). 


\subsubsection{Cytokeratins-7 and -19 (CK-7 and CK-19)}

Cytokeratins are the largest subfamily of intermediate filament proteins and include more than 20 different gene products. Different epithelial types are characterized by the expression of specific cytokeratin (CK) subtypes. Cytokeratins can therefore serve as a 'lineage marker' of epithelial cells. In the liver CK-7 and CK-19 are the two cytokeratins expressed exclusively in the intrahepatic and extrahepatic bile duct epithelial cells. They are known to undergo extensive changes in expression with alteration of the hepatocyte phenotype in vitro (Sasaki et al., 2001; Van Eyken et al., 1987; Saunders et al., 2000).

The canals of Hering $(\mathrm{CoH})$ begin in the lobules, are lined partially by cholangiocytes and partly by hepatocytes, and conduct bile from bile canaliculi to terminal bile ducts in portal tracts. They are not readily apparent on routine histological staining but are highlighted by the biliary cytokeratins CK-19 and CK-7. The canals represent the true hepatocyticbiliary interface that lies within the lobule and not at the limiting plate. The $\mathrm{CoH}$ are destroyed early in primary biliary cirrhosis, perhaps explaining lobular "hepatitis" in this disease. They may also be the primary sites of scarring in methotrexate toxicity. Most intriguingly, the $\mathrm{CoH}$ have been speculated to harbor intraorgan stem cells of the liver, perhaps forming the hepatic stem cell "niche" and have been demonstrated to proliferate in disease states (Saxena and Theise, 2004).

\subsection{Background}

During rat embryogenesis the liver bud begins to develop at 10 days of gestation (E10), when the foregut and hindgut become visible followed by a connection of the foregut endoderm with the developing heart that leads to initiation of hepatic specification of the ventral foregut endoderm. Much 
is known about how signaling molecules such as bone morphogenetic proteins (BMPs), fibroblast growth factors (FGFs) and transcription factors such as GATA-4, Foxa-1 and Foxa-2 induce the liver development (Zaret, 1996); Zhao et al., 2005; Serls et al., 2005; Deutsch et al., 2001). The characterization of endodermal cells generated from ventral foregut which are involved in this process, has been neglected. In addition, the earliest embryonic developmental stage for the emergence of hepatoblasts that can express albumin and AFP remains controversial.

\subsubsection{Kinetics for albumin and AFP mRNA expression during liver development.}

Albumin and alpha-fetoprotein represent the main hepatic markers. Densitometric analysis after hybridization with albumin and AFP cDNA probes revealed that albumin and AFP mRNA could not be detected in the foregut region in rat embryos (Muglia and Locker, 1984). In contrast, AFP mRNA was clearly detected by in situ histological analysis in the ventral endoderm at E10.5 (10.5 days of gestation). At E11.5 both AFP mRNA and its coding protein were present in hepatoblasts. At this stage albumin mRNA was also identified but its protein product appeared one day later (at E12.5) (Shiojiri et al., 1991).

Recent experiments in mouse embryos from E8-E9.5 (corresponding to E9.5-E11 in the rat) provided much insight into albumin and AFP gene expression. Albumin becomes detectable by immunofluorescence in sectioned embryos from 9-10 somites stages as well as in single cell cytospin slides generated from dissected ventral foregut endoderm at 8 , 10 and 14 somites (Serls et al., 2005). Tissue explants generated from ventral endoderm region at 4 to 6 somites stages have revealed that AFP mRNA is expressed in the ventral and dorsal endoderm. In contrast, albumin mRNA expression is induced through a co-culture with cardiac mesoderm (Gualdi et al., 1996) or after stimulation with different types, 
and concentration of fibroblast growth factors (FGFs) (Jung et al., 1999; Deutsch et al., 2001); (Sekhon et al., 2004).

The embryonic liver appears on day 12 of gestation, the day when albumin and AFP mRNA as well as their corresponding proteins are expressed. It has been demonstrated that the expression of albumin and AFP transcripts change during liver development. AFP mRNA reaches a peak at 16 days of gestation and then decreases, while albumin mRNA reaches a peak at 18 days of gestation and persists at the following stages (Muglia and Locker, 1984). In other terms, it has been claimed that albumin gene expression increases gradually during liver development, and the ratio of AFP synthesis to albumin synthesis as well as the ratio of AFP mRNA to albumin mRNA suggests a gradual decrease during liver development (Petkov et al., 2004); Liao et al., 1980).

\subsubsection{Distribution of albumin and AFP producing cells during liver development}

During liver development albumin and AFP producing cells (hepatoblasts) undergo a proliferation process that leads to an increase in liver size (Micsenyi et al., 2004). It has been demonstrated using foetuses from 17 to 19 days of gestation and newborn animals that albumin and AFP genes are expressed simultaneously by all hepatocytes. However, in the first weeks of postnatal life albumin and alpha-fetoprotein genes are highly expressed by periportal and perivenous hepatocytes respectively (Poliard et al., 1986).

\subsubsection{Synthesis and secretion of albumin and AFP}

Albumin and AFP as secretory proteins are in general synthesized on polysomes bound to the rough endoplasmic reticulum (RER) and cotranslationally transferred to the lumen of this compartment. Proteins 
are then transported from ER to the Golgi apparatus and secreted. (Lodish et al., 1983) and (Fries et al., 1984) have demonstrated it as a rate limiting step. The kinetics for the intracellular transfer of apoB-100 in comparison to albumin and transferrin was investigated in hepatoma cell-line HepG2 by using a pulse chase methods combined with subcellular fractionation (Bostrom et al., 1986).

\subsubsection{Cytokeratin-7 and -19 expression during intrahepatic bile duct development}

In several studies, the bile duct development is based on the expression of the main bile duct marker (cytokeratin-7 and -19). The early hepatoblasts are supposed to be bipotent, giving rise to hepatocytes and intrahepatic cholangiocytes. Intrahepatic bile ducts start to differentiate from periportal hepatoblasts, which express AFP and albumin at embryonic day E15.5 in the rat, some of those cells were CK-19 positive and AFP negative (Shiojiri et al. 1991). Cytokeratin-7 (CK-7) is expressed in biliary epithelial cells in the late gestational stage (Shiojiri et al. 1991; Pack et al., 1993). It has been claimed that in foetal rat liver, cells expressing cytokeratin-19 appeared at 17 and 18 days of gestation. However, the expression of cytokeratin-7 begins later than that of cytokeratin-19 and it is present only in cholangiocytes throughout pre- and postnatal ontogenesis (Kiiasov et al., 1997).

(Gall and Bhathal, 1989) have demonstrated that intrahepatic bile ducts develop by a reorganization and modulation of the periportal hepatoblasts to biliary epithelial cells (BEC). They thought that bile duct development takes place in the 19 day foetus around the larger branches of the portal vein, with the formation of lumina surrounded by cuboidal or elongated hepatoblast-like cells on the portal aspect and readily distinguished hepatoblasts on the lobular aspect. On 21 day of gestation these 
structures had developed into canals of Hering lined jointly by recognizable liver cells and BEC. The number of canals of Hering per portal tract peaked on 22 day of gestation and diminished in number at birth. Bile ducts lined completely by BEC were first found on 20 day of gestation. At this developmental stage prekeratin antigens were first detected in duct-like structures not only in phenotypic BEC but also in adjacent cells with a hepatoblast phenotype. Such intermediate cells were present until birth.

\subsubsection{Cytokines regulating hematopoiesis in embryonic and adult state}

Hematopoiesis is controlled by different regulators, namely granulocytemacrophage colony-stimulating factor (GM-CSF), granulocyte colonystimulating factor (G-CSF), stem cell factor (SCF) and Erythropoietin (Epo). GM-CSF and SCF play regulatory functions in the early steps of erythropoiesis (Chui and Loyer, 1975; Pillarisetty et al., 2003; Sansone et al., 1979; Praloran, 1991). GM-CSF enhances the differentiation of granulocyte. This GM-CSF mediated differentiation is associated with the up-regulation of G-CSF receptor mRNA (Collins et al., 2001).

Erythropoietin (Epo) is the main factor which acts positively on the last steps of the production of erythrocytes. It is specific for the erythroid progenitor cells and has only little effect on other cells. Erythropoietin acts on these progenitors through surface receptors specific for Erythropoietin and induces the proliferation and differentiation of erythroid progenitors leading finally to reticulocytes (Johnson and Barker, 1985; Wong et al., 1983). Macrophages play a supportive role in erythropoiesis. Erythroid colony-forming units adhere to a macrophage and differentiate to erythroblasts in the presence of erythropoietin (Epo), resulting in the formation of an erythroblastic island (Sasaki et al., 1993). 


\subsection{Objective of the work}

The aim of this study is the characterization of embryonic/foetal liver cells during liver development, from the earliest stage of liver development (the time of hepatic specification) up to adulthood. Compared to mature liver, the embryonic/foetal liver were characterized as follows:

1) Cultured endodermal cells derived from ventral foregut region were used to investigate albumin and AFP synthesis and secretion. Radioactive biosynthetic labelling proved to be a very sensitive method for this purpose. The endodermal cells derived from ventral foregut region were further characterized by measuring the expression of hepatic and endodermal markers.

2) Cellular analysis was performed on the developing liver. The ratios of albumin and AFP expressing cells to both total liver cells and to proliferating cells were measured at all developmental stages.

3) The kinetics of synthesis and secretion of albumin and AFP in hepatoblasts at all developmental stages was assessed, with a view to estimating the secretion-velocity and to see whether the secretion apparatus works as in adult liver.

4) Hepatoblasts from 12 and 14 days of gestation were passaged three times in order to determine if their hepatic character was lost during the passage. For this purpose the expression of albumin and AFP was measured after each passage.

5) Hepatoblasts derived from embryonic and fetal stages were stimulated with interleukin-6 (IL-6), after which the synthesis and secretion of albumin 
and AFP were examined. The expression level of interleukin-6 receptor mRNA (IL-6rec) was also measured in unstimulated hepatoblasts.

6) The bile duct development was investigated by assessing the in vivo and in vitro gene expression of prox 1 , cytokeratin- 7 and -19 during liver development.

7) The expression of cytokines (granulocyte-macrophage colonystimulating factor (GM-CSF), granulocyte colony-stimulating factor (G$\mathrm{CSF}$ ), stem cell factor (SCF) and Erythropoietin (Epo) involved in the regulation of hematopoiesis was also examined during liver development. 


\section{MATERIALS AND METHODS}

\subsection{Animals}

Pregnant Wistar rats (Rattus norvegicus, Sprague Dawley) were purchased from Harlan Winkelmann (Borchen, Germany). The rats were prepared on the same day of delivery or kept at $19-23^{\circ} \mathrm{C}$ under standard conditions with 12-hour light/dark cycles and access to fresh water and till desired development stage achieved. The Rats were anesthetized by intraperitoneal injection of pentobarbital (400 mg/kg body weight). All animals received humane care in accordance with the institution's guidelines, the German Convention for Protection of Animals and the National Institutes' of Health guidelines. In this study about 400 pregnant rats were used, corresponding to 5500 - 6000 embryos.

\subsection{Chemicals, Enzymes and Kits}

All chemicals, Enzymes and Kits used in this study are listed below.

Agarose

Amplify $^{\mathrm{TM}}$ Fluorographic Reagent

Acetone

Ammonium persulfate (APS)

Acetic acid

Ampuwa water

Acrylamid/Bisacrylamid

Bromophenol blue
Invitrogen, Karlsruhe

GE Healthcare, UK

Merck, Darmstadt

Merck, Darmstadt

Merck, Darmstadt

Fresenius Kabi, Homburg

Sigma, München.

Sigma, München

5-bromo-4-chloro-3-indolyl-phosphate (BCIP) Roche, Mannheim 
Blocking reagent (DIG Nucleic Acid

Detection Kit)

BSA (bovine serum albumin)

3-[(3-Cholamidopropyl)dimethylammonio] -1-propanesulfonate (CHAPS)

Cesium chloride $\left(\mathrm{CsCl}_{2}\right)$

Calcium chlorid $\left(\mathrm{CaCl}_{2}\right)$.

Collagen type I

Citric acid -monohydrate

Collagenase $\mathrm{H}$

DAB (3,3`-Diaminobenzodine)

Dexametasone

$\mathrm{N}-\mathrm{N}$-Dimethylformamide (DMF).

Dextran sulfate sodium salt

Digoxygenine-U-dNTP

Dihydroxyaceton

DMEM medium

(Diethylpyrocarbonat) DEPC

Dithiothreitol (DTT)

DNase I (10000 Units)

DNA Molecular Weight Marker XVII, 500bp Ladder

Deoxycholic acid (DOC)

Ethidiumbromide

(Ethylenediaminetetraacetic acid) EDTA

Ethanol absolut p.A.

80\% Ethanol techn.(Alkopharm)

Fetal calf serum (FCS)
Roche, Mannheim

Serva, Heidelberg

Biorad, München

Invitogen, Karlsruhe

Merck, Darmstadt

Becton Dickinson (Bedfort, USA)

Sigma, München

Boehringer Mannheim, 1074032

Sigma, München

Sigma, München

Carl Roth, Karlsruhe

GE Healthcare, UK

Sigma, München

Sigma, München

PAA, Pashing

Sigma, München

Sigma, München

Boehringer Mannheim

Boehringer, Mannheim

Sigma, München

Sigma, München

Merck, Darmstadt

Merck, Darmstadt

BrüggemannAlcohol, Heilbronn

Sigma, München 
Formamide

Fastlane Cell cDNA kit

Formaldehyd $37 \%$

Fluoromount-G

Full Range Rainbow protein molecular

weight marker RPN 800

Fluorescein-U-dNTP

D-Glucose

Glucagon

L-Glutamine

Guanidinthiocyanate (GITC)

Glycine

Glutaraldehyde

Glucoseoxidase

$10 \%$ goat serum

$\mathrm{HCl}(5 \mathrm{~N})$

HEPES

Hydrogen peroxide $30 \%\left(\mathrm{H}_{2} \mathrm{O}_{2}\right)$

HybridoMed DIF 1000 medium

Herings-Sperm DNA

Rat Interleukin-6(IL-6)

Insulin

Isopropanol

Kaisers-Glyceringelatine

Levamisol

6X Loading Dye Solution

Mixed Bed Resin, AG 501-X8 (D)

Minimal essential medium (MEM) Hanks

standard with stable Glutamine
Merck, Darmstadt

Qiagen, Hilden

Merck, Darmstadt

Southern Biotechnology

GE Healthcare, UK

Roche, Mannheim

Merck, Darmstadt

Sigma, München

Fulka, Buchs Schweiz

Invitrogen, Karlsruhe

Sigma, München

Carl Roth, Karlsruhe

Sigma, München

DAKO, Glostrup, Denmark

Merck, Darmstadt

Sigma, München

Merck, Darmstadt

Biochrom KG, Berlin

Roche, Mannheim

Pepro Tech Inc, NJ USA

Sigma, München

Merck, Darmstad

Merck, Darmstadt

Sigma, München

MBI Fermentas

Bio-Rad, München

Promocell, Heidelberg 
Magnesium chloride $\left(\mathrm{MgCl}_{2}\right)$

L-methionine

$\left({ }^{35} \mathrm{~S}\right)$ Radioaktives methionine

Methanol

$\beta$-Mercaptoethanol

Mayers Hemalaun solution

M-MLV Reverse Transcriptase (RT) Kit

$5 x$ first strand buffer

DTT Dithiothreitol

Oligo (dT)12-18 (500 $\mu \mathrm{g} / \mathrm{ml})$

dNTP Mix ( $10 \mathrm{mM}$ each dATP,

dGTP, dCTP and dTTP)

MMLV RT

Naphthol AS-BI-phosphate disodium salt (Na-As-Bi-P)

Narcoren

Nitroblue tetrazolium salt (NBT)

$\mathrm{N}, \mathrm{N}, \mathrm{N}^{\prime}, \mathrm{N}^{\prime}$-Tetramethylethylenediamine (TEMED)

New fuchsin

Qiagen-II-Gel-Extraction-Kit

PBS without $\mathrm{Ca}++, \mathrm{Mg}++$

Taq PCR Master Mix

Phenylmethylsulfonyl Fluoride (PMSF)

Penicillin/Streptomycin

Paraformaldehyde (PFA)

Propandiol

Proteinase $\mathrm{K}$
Merck, Darmstadt

Sigma, München

GE Healthcare, UK

Merck, Darmstadt

Merck, Darmstadt

Merck, Darmstadt

In vitrogen, Karlsruhe

In vitrogen, Karlsruhe

In vitrogen, Karlsruhe

In vitrogen, Karlsruhe

In vitrogen, Karlsruhe

In vitrogen, Karlsruhe

Sigma, München

Merial, Hallbergmoos

Roche, Mannheim

Sigma, München

Sigma, München

Qiagen, Hilden

Biochrom KG, Berlin, Germany

Qiagen, Hilden

Sigma, München

Sigma, München

Merck, Darmstadt

Merck, Darmstadt

Roche, Mannheim 
Protein A

Potassium Chloride $(\mathrm{KCl})$

Potassium dihydrogen phosphate $\left(\mathrm{KH}_{2} \mathrm{PO}_{4}\right)$

Rnase inhibitor

RNase A

RPMI medium (Methionine-free)

Scintillation liquid

Sodium Dodecyl Sulfate (SDS)

Sarcosyl (N-Laurylsarcosine

Sodium-Acetate

Sodium citrate

Sodium Chloride $(\mathrm{NaCl})$

Sodiumhydroxid $(\mathrm{NaOH})$ pellets.

Sodium azide

di-sodium hydrogen phosphate $\left(\mathrm{Na}_{2} \mathrm{HPO}_{4}\right)$

Sodiumhydroxide $(5 \mathrm{~N})$

Sodiumnitrite (NaNO2)

SYBR ${ }^{\circledR}$ Green PCR Mastermix

tRNA yeast

TRIS-HCl

TRIS-Base

T7 RNA Polymerase (5000 Units)

Triton X-100

Tri-sodium citrate

Trichloracetic acid (TCA)

Trypan blue

Tissue.Tek O.C.T. ${ }^{\mathrm{TM}}$ Compound

10 x Trypsin - EDTA
Roche, Mannheim

Merck, Darmstadt

Merck, Darmstadt

Roche, Mannheim

Roche, mannheim

Biochrom KG, Berlin

Zinsser Analytic, Francfurt

Karl Roth, Karlsruhe

Sigma, München

Sigma, München

Sigma, München

Merck, Darmstadt

Merck, Darmstadt

Merck, Darmstadt

Merck, Darmstadt

Merck, Darmstadt

Merck, Darmstadt

Invitrogen, Karlsruhe

Invitrogen, Karlsruhe

Serva, Heidelberg

Carl Roth, Karlsruhe

Roche, Mannheim

Sigma, München

Merck, Darmstadt

Merck, Darmstadt

Sigma, München

SakuraFinetek, Zoeterwounde NL

Gibco (Karlsruhe, Deutschland) 
Polyoxyethylenesorbitan monolaurate

(Tween ${ }^{\circledR 20}$ )

William's E Medium

\subsection{Antibodies}

Anti-alpha-Fetoprotein rabbit polyclonal

Anti-albumin antibody rabbit polyclonal

Anti-cytokeratin (CK-7) mouse monoclonal

Anti-connexin 32 mouse monoclonal

Anti- cytokeratin (CK-19) mouse monoclonal

Anti-HepPar1 mouse monoclonal

Anti-Dioxygenine-alkaline

phosphatase conjugated (Anti-DIG-AP)

Anti-Fluorescein-alcaline

phosphatase conjugated (Anti-FL-AP)

Anti-NF4alpha antibody rabbit polyclonal

Anti-proliferating cell nuclear antigen

antibody (anti-PCNA) mouse monoclonal

Anti-Prox1 antibody rabbit polyclonal

Anti-mouse horseradish peroxidase

(HRP)-conjugated antibody

Alexa 555-conjugated secondary

anti-rabbit antibodies

Alexa 488-conjugated secondary

Anti-mouse antibodies

4'-6-Diamidino-2-phenylindole (DAPI)
Sigma, München

Biochrom KG, Berlin
Dako, Glostrup, Denmark

Dako, Glostrup, Denmark

Dako, Glostrup, Denmark

Alpha Diagnostic Int, USA

Novocastra, UK

Dako, Glostrup, Denmark

Roche, Mannheim

Roche, Mannheim

Santa Cruz, California

Novocastra, UK

Strathmann, Hamburg

Dako, Glostrup, Denmark

Molecular Probes,

Leiden, Netherlands

Molecular Probes,

Leiden, Netherlands

Molecular

Probes, Leiden, Netherlands 


\subsection{Oligonucleotides}

General PCR reactions for preparation of riboprobes used for in situ hybridization and for quantitative analysis of genes expression were carried out with sequence-specific primers from Invitrogen (Karlsruhe,

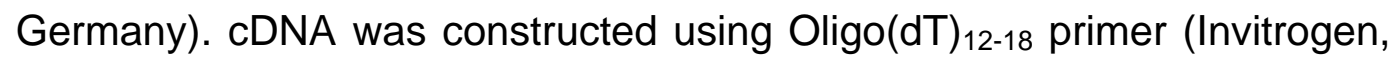
Karlsruhe, Germany). All primers used are listed below.

\begin{tabular}{|l|l|}
\hline Primer & Sequence \\
\hline Antisense AFP for & GGCGATGTCCATAAACACGTTC \\
\hline Antisense AFPT7 rev & TAATACGACTCACTATAGGGCCGGTTTGTCGCCATT \\
\hline Sense AFPT7 for & $\frac{\text { TAATACGACTCACTATAGGGGGCGATGTCCATAAAC }}{\text { ACGTTC }}$ \\
\hline Sense AFP rev & CCGGTTTGTCGCCATTTTC \\
\hline Antisense Alb for & GGATTCCAAAACGCCGTTCT \\
\hline Antisense AlbT7 rev & $\begin{array}{l}\text { TAATACGACTCACTATAGGGCCTCAGTGGCGAAGC } \\
\text { SGTTATC }\end{array}$ \\
\hline Sense AlbT7 for & TAATACGACTCACTATAGGGGGATTCCAAAACGCCG \\
\hline Sense Alb rev & CCTCAGTGGCGAAGCAGTTATC \\
\hline T7 Promoter & $\underline{\text { TAATACGACTCACTATAGGG }}$ \\
\hline
\end{tabular}

Table1: List of primers used for preparation of albumin and AFP sense and antisense (For $=$ Forward, rev $=$ reverse). When $\mathrm{T} 7$ promoter (underlined sequence) is upstream of the reverse primer, we obtain a cDNA construct for the synthesis of an antisense probe. When a T7 promoter is upstream of the forward primer, we generate a cDNA construct for the synthesis of sense probe 
Table 2: Primers used for quantitative analysis by real-time PCR analysis (For = Fotward, Rev = reverse)

\begin{tabular}{|l|l|}
\hline Primer & Sequence \\
\hline Albumin For & GGATTCCAAAACGCCGTTCT \\
\hline Albumin Rev & CCTCAGTGGCGAAGCAGTTATC \\
\hline AFP For & GGCGATGTCCATAAACACGTTC \\
\hline AFP Rev & CCGGTTTGTCGCCATTTTC \\
\hline HNF4alpha For & CTT CTT TGA CCC AGA TGC CAA G \\
\hline HNF4alpha Rev & GCC GGT CGT TGA TGT AAT CCT \\
\hline Beta-catenin For & CGC ACC ATG CAG AAT ACA AAT G \\
\hline Beta-cateninRev & GGA TGC CGC CAG ATT TAA AGA T \\
\hline Prox1 For & GCTCCAATATGCTGAAGACC \\
\hline Prox1 Rev & ATCGTTGATGGCTTGACGTG \\
\hline BMP4 For & TTCCCTCAAGGGAGTGGAAATTC \\
\hline BMP4 Rev & CCATCGTGGCCAAAAGTGA \\
\hline GATA-4 For & TTG ATC TCC GTT TTC GCG AC \\
\hline GATA-4 Rev & GCT CCC CTT TAT TTG CAA GTC A \\
\hline Foxa2 For & CAT GGT GAA ATC CAG GTC TCG \\
\hline Foxa2 Rev & TGG AAC TCT GGC ATT CTA GCC \\
\hline Interleukin receptor For & TTGCAATTCGAGCTTCGATACC \\
\hline Interleukin receptor Rev & TCGCAAGGCATCATGGATG \\
\hline Ribosomal 18S For & CGGCTACCACATCCAAGGAA \\
\hline Ribosomal 18S Rev & TTTTCGTCACTACCTCCCCG \\
\hline GAPDH For & TCC TGC ACC ACC AAC TGC TTA G \\
\hline GAPDH Rev & TTC TGA GTG GCA GTG ATG GCA \\
\hline GM-CSF For & GGCSF Rev \\
\hline
\end{tabular}




\begin{tabular}{|l|l|}
\hline SCF For & AACCTGCAGCCCGTAGTTTA \\
\hline SCF Rev & AGTGGCTGATGCTACGGAGT \\
\hline Erythropoietin For & TCCCACCCTGCTGCTTTTACT \\
\hline Erythropoietin Rev & CCCATTGTGACATTTTCTGCC \\
\hline
\end{tabular}

\subsection{Other Materials}

Dako pen, Dako, Glostrup, Denmark

24-well plates, Petri dishes (100 mm), Greiner, Frickhausen, Germany

6-well plates, 96-well microtiter plates, Lab-Tek chamber slides, Nunc, Naperville, IL,USA

Cover-slips, 24x55 mm, Menzel-Gläser, Braunschweig, Germany

Culture dishes (35, 100 and $150 \mathrm{~mm}$ ), Becton Dickinson Labware, Lincoln

Park, NJ, USA

Intravenous cannula with injection port, Braun, Melsungen, Germany

Intravenous cannula with injection port, Klinika Medical, Usingen,

Germany

Latex powder-free gloves, Kimberly-Clark, Zaventem, Belgium

Microscope glass slides, 76×26 mm, Menzel-Gläser, Braunschweig,

Germany

Nitrile gloves Nitra Tex, Ansell, Kulim, Malaysia

Nitrocellulose Transfer Membrane, Sartorius, Göttingen, Germany

Polyallomer thin-walled centrifuge tubes ( $5 \mathrm{ml}$ ), Beckman, Munich,

Germany

Safe-Lock tubes (0.2, 0.5, 1.5 and $2 \mathrm{ml})$, Eppendorf, Hamburg, Germany

Scintillation vials ( $5 \mathrm{ml})$, Zinsser Analytic, Frankfurt, Germany

Serological pipettes $(2,5,10,25 \mathrm{ml})$, transfer pipettes, plastic tubes (15 and $50 \mathrm{ml}$ ),

Sarstedt, Nümbrecht, Germany 
Sterile filter Nalgene, $0.2 \mu \mathrm{m}$, Sartorius, Göttingen, Germany

Sterile filter pipette tips, Biozym, Oldendorf, Germany

Whatman 3MM paper, Schleicher and Schuell, Dassel, Germany

X-ray films HyperfilmTM, Amersham Biosciences, Freiburg, Germany

X-ray films X-Omat AR, Kodak, Rochester, NY, USA

X-ray films, Fuji, Düsseldorf, Germany

X-ray films, Konica, Hohenbrunn, Germany

\subsection{Instruments}

Automatic pipettes, type Reference ${ }^{\circledR}$, Eppendorf, Hamburg, Germany

Automatic pipettes, type Pipetman, Gilson, Bad Camberg, Germany

Bench-top centrifuges, high speed centrifuges, ultracentrifuges and rotors:

Beckman model J2-21 centrifuge

Beckman, Munich,

Beckman rotor JE-6B

Germany

Centricon T-2070 ultracentrifuge

Kontron Instruments

Centricon rotor TST55.5 - 55000 rpm

, Neufahrn, Germany

Eppendorf bench-top centrifuge,

Eppendorf, Hamburg

type MiniSpin 5415C

Germany

Hettich Mikro Rapid/K centrifuge

Hettich Rotina 3850 centrifuge

Hettich, Tuttlingen, Germany

Hettich Rotina 48RS centrifuge

Hettich Rotixa/RP centrifuge

Dissection tools: Micro-Dissecting

Martin Surgery, Tuttlingen

Scissors, Micro-Forceps

Minifuge GL centrifuge

Heraeus-Christ, Osterode, Germany 
Sigma 3K30 centrifuge

Rotor Nr 12156 - 16500 rpm

Rotor Nr 12153 - 22000 rpm

Sigma Laboratory Centrifuges

Osterode, Germany

Digital photocamera Canon EOS D60 and software Remote Capture 2.5,

Canon, Tokyo, Japan

Eagle Eye ${ }^{\mathrm{TM}}$ system with built-in ultraviolet emitter, video camera and frame integrator, Stratagene, Amsterdam, Netherlands

Electrophoresis power supply Power Pac 300, Bio-Rad, Munich, Germany Electrophoresis power supply ST305, Invitrogen, Karsruhe, Germany

End-over-end rotator, W.Krannich, Göttingen, Germany

Gamma-counter, Mini-instruments, Burnham-on-Crouch, UK

Gas controlled incubators, Heraeus-Electronic, Hannover, Germany

Geiger hand gamma-counter, Berthold, Oak Ridge, TN, USA

Gel dryer, LKB Bromma, Bromma, Sweden

Hybridization oven, Biometra, Göttingen, Germany

Ice machine, Ziegra, Isernhagen, Germany

Liquid scintillation counter Wallac 1409, EG\&G, Turku, Finland

Magnetic mixer with warming, type M21/1 Framo-Gerätetechnik,

Eisenach, Germany

Microplate reader MRX, Dynatech Laboratories, Chantilly, VA, USA

Microscope Axioscop with fotocamera MC 100 Spot, Zeiss, Oberkochen, Germany

Microscope Axiovert 25, Zeiss, Oberkochen, Germany

Microwave, Whirlpool, Comerio, Italy

Mini-vertical gel electrophoresis unit Hoefer Mighty Small II, Amersham

Biosciences, Freiburg, Germany

Peristaltic pump P-1, Amersham Biosciences, Freiburg, Germany

pH-Meter, Glas-Gerätebau, Bovenden, Germany

Pipette holder with safety valve, filter and wall holder; Hirschmann

Laborgeräte, Eberstadt, Germany

Rocking platform, Biometra, Göttingen, Germany 
Savant Speed Vac ${ }^{\circledR}$ concentrator, ThermoLife Sciences, Egelsbach, Germany

Scanning densitometer, Molecular Analyst, Bio-Rad Hercules, CA, USA

Sonicator Sonoplus HD 70, Bandelin, Berlin, Germany

Standard dual cooled gel electrophoresis unit Hoefer SE 600, Amersham

Biosciences, Freiburg, Germany

Sterile bench, The Baker Company, Sanford, ME, USA

Thermomixer 5436, Eppendorf, Hamburg, Germany

Thermostat, Heraeus, Hanau, Germany

Thermostatic calculator, model 2219 Multitemp II, LKB Bromma, Bromma, Sweden

Transfer electrophoresis unit Hoefer TE 50X, Amersham Biosciences, Freiburg, Germany

Transfer electrophoresis unit Mini Trans-Blot ${ }^{\circledR}$, Bio-Rad, Munich, Germany

Ultra-pure water system Milli-Q, Millipore, Molsheim, France UV spectrophotometer, RNA/DNA Calculator GeneQuant II, Pharmacia Biotech, Freiburg, Germany

Vortex, Genie $2^{\mathrm{TM}}$, Bender and Hobein, Zurich, Switzerland

Vortex, with platform, Schütt Labortechnic, Göttingen, Germany

Water bath, W.Krannich, Göttingen, Germany

X-ray film cassettes $10 \times 18$, Siemens, Munich, Germany

X-ray film-developing machine SRX-101A, Konica Europe, Hohenbrunn, Germany 


\subsection{Embryos, hepatoblasts isolation and culture}

\subsubsection{Dissection of the embryos}

Pregnant rats from different gestation stages were prepared under Narcoren anesthesia (pentobarbital (400 mg/kg body weight). The uterus was removed and placed in Petri dish with cold PBS (with $\mathrm{Ca}^{++}$and $\mathrm{Mg}^{++}$). The embryos from E10, E12, E14, E16, E18 and E20 were removed, collected in ice-cold minimal essential medium (MEM) Hank's standard with glutamine (preparation-medium I). The ventral foregut endoderm from embryos at E10 and the liver from embryos at E12 and E14 were dissected under binocular microscope and incubated in MEM Hanks medium with $25 \mathrm{mM}$ Hepes, stable glutamine supplemented with $0.05 \%$ DNase (preparation-medium II). The liver from E16, E18, E20 and new born were dissected macroscopically and collected in 1ml MEM Hanks with $25 \mathrm{mM}$ Hepes, stable glutamine supplemented with 0.05\% DNase and $0.05 \%$ collagenase $\mathrm{H}$ (Preparation-Medium III). A single cell suspension was obtained after two times incubation at $37^{\circ} \mathrm{C}$ for 15 min followed by several times pipeting with a melted $1 \mathrm{ml}$ and $100 \mu$ pipet tip. Cells were collected after centrifugation ( $243 \mathrm{~g}, 5 \mathrm{~min}, \mathrm{RT}$ ). Cell viability was assessed by trypan blue staining and cell number was determined in a Neubauer chamber. Cells were diluted in William's $E$ medium supplemented with $10 \%$ foetal calf serum, $100 \mathrm{U} / \mathrm{ml}$ penicillin, $100 \mu \mathrm{g} / \mathrm{ml}$ streptomycin, $1 \%$ L-glutamine, $0.1 \mu \mathrm{g} / \mathrm{ml}$ insulin, $0.1 \mu \mathrm{g} / \mathrm{ml}$ glucagon and $0.1 \mu \mathrm{g} / \mathrm{ml}$ dexamethasone. Subsequently cells were seeded onto collagen type I coated 24 well plate or lab-tecks and incubated at $37^{\circ} \mathrm{C}$ humidified $5 \% \mathrm{CO} 2$ incubator. After $24 \mathrm{~h}$ the cells were washed twice with PBS and kept in culture in serum free HybridoMed medium (DIF) supplemented with $100 \mathrm{U} / \mathrm{ml}$ penicillin, $100 \mu \mathrm{g} / \mathrm{ml}$ streptomycin, 1\% L-glutamine, 0.1 $\mu \mathrm{g} / \mathrm{ml}$ insulin, $0.1 \mu \mathrm{g} / \mathrm{ml}$ glucagon and $0.1 \mathrm{ug} / \mathrm{ml}$ dexamethasone. 


\subsubsection{Medias}

\section{Preparation-Medium I:}

Minimal Essential Medium (MEM) Hanks standard with stable glutamine

\section{Preparation-Medium II:}

Minimal Essential Medium (MEM) Hanks with 25 mM HEPES and stable glutamine, supplemented with $0.05 \%$ DNase

\section{Preparation-Medium III:}

Minimal Essential Medium (MEM) Hanks with 25 mM HEPES and stable glutamine, supplemented with $0.05 \%$ DNase and $0.05 \%$ Collagenase $\mathrm{H}$

\section{WE with $10 \%$ FCS culture medium:}

William's E Medium (1x) with

$10 \%$ fetal calf serum (FCS)

Dexamethasone $(0.1 \mu \mathrm{g} / \mathrm{ml})$

Glucagon $(0.1 \mu \mathrm{g} / \mathrm{ml})$

Insulin $(0.1 \mu \mathrm{g} / \mathrm{ml})$

$100 \mathrm{U} / \mathrm{ml}$ penicillin

$100 \mu \mathrm{g} / \mathrm{ml}$ streptomycin

1\% L-glutamine

\section{DIF culture medium:}

HybridoMed DIF 1000 (1x) with

Dexamethasone $(0.1 \mu \mathrm{g} / \mathrm{ml})$

Glucagon $(0.1 \mu \mathrm{g} / \mathrm{ml})$

Insulin $(0.1 \mu \mathrm{g} / \mathrm{ml})$

$100 \mathrm{U} / \mathrm{ml}$ penicillin

$100 \mu \mathrm{g} / \mathrm{ml}$ streptomycin

1\% L-glutamine 


$\begin{array}{ll}\text { PBS-Buffer (with } \mathbf{~ M g}^{2} \\ \mathrm{NaCl} & 137 \mathrm{mM} \\ \mathrm{KCl} & 2.7 \mathrm{mM} \\ \mathrm{Na}_{2} \mathrm{HPO}_{4} & 7.5 \mathrm{mM} \\ \mathrm{KH}_{2} \mathrm{PO}_{4} & 1.47 \mathrm{mM} \\ \mathrm{CaCl}_{2} & 1 \mathrm{mM} \\ \mathrm{MgCl} & 0.5 \mathrm{mM}\end{array}$

The $\mathrm{pH}$ of PBS-Buffer was set up to 7.4 with $\mathrm{HCl}$. Finally, the buffer was filtered through 0,45 $\mu \mathrm{m}$ Stericup (Millipore) under sterile conditions and stored at $4{ }^{\circ} \mathrm{C}$.

\subsection{Primary hepatocyte isolation and culture}

\subsubsection{Isolation and culture}

Hepatocytes were isolated from Wistar rats by a perfusion technique as described elsewhere (Ramadori et al., 1990). Briefly, the liver was shortly perfused with a calcium-free saline (Buffer I) and then with a solution

containing $0.05 \%$ collagenase $\mathrm{H}$ (Buffer II). The liver was removed and cell suspension was collected in Buffer III. Hepatocytes were seperated from non-parenchymal cells by centrifugation at $243 \mathrm{~g}$ for $5 \mathrm{~min}$ at $4^{\circ} \mathrm{C}$. Cell viability was assessed by trypan blue staining and the cell number was determined in a Neubauer chamber. The hepatocytes $\left(5 \times 10^{4}\right.$ cells $/ \mathrm{cm}^{2}$ ) were plated onto collagen type l-coated 24 well plates or labtecks in DMEM medium suplemented with $4.5 \mathrm{~g} / \mathrm{l}$ glucose $10 \%$ fetal calf serum, $100 \mathrm{U} / \mathrm{ml}$ penicillin, $100 \mu \mathrm{g} / \mathrm{ml}$ streptomycin, 1\% L-glutamine, 0.1 $\mu \mathrm{g} / \mathrm{ml}$ insulin, $0.1 \mu \mathrm{g} / \mathrm{ml}$ glucagon and $0.1 \mu \mathrm{g} / \mathrm{ml}$ dexamethasone. After $2 \mathrm{~h}$, the medium was changed and the hepatocytes were kept in culture at $37^{\circ} \mathrm{C}$ in a humidified $5 \% \mathrm{CO} 2$ incubator. 


\subsubsection{Buffers}

All solutions for hepatocyte isolation were prepared in double distilled water and sterile filtered through $0.45 \mu \mathrm{m}$ Stericup (Millipore) and stored at $4{ }^{\circ} \mathrm{C}$.

\section{Buffer I}

$\mathrm{NaCl}$

$8.3 \mathrm{~g} / \mathrm{l}$

$\mathrm{KCl}$

$0.5 \mathrm{~g} / \mathrm{l}$

HEPES

$\mathrm{pH}$ was adjusted to 7.4 with $\mathrm{HCl}$

\section{Buffer II}

$\begin{array}{ll}\mathrm{NaCl} & 3.9 \mathrm{~g} / \mathrm{l} \\ \mathrm{KCl} & 0.5 \mathrm{~g} / \mathrm{l} \\ \mathrm{CaCl}_{2} 2 \mathrm{H}_{2} \mathrm{O} & 0.7 \mathrm{~g} / \mathrm{l} \\ \mathrm{HEPES} & 24 \mathrm{~g} / \mathrm{l}\end{array}$

$\mathrm{pH}$ was adjusted to 7.6 , before use $0.05 \%$ Collagenage $\mathrm{H}$ was added

\section{Buffer III}

$\begin{array}{ll}\mathrm{NaCl} & 8.3 \mathrm{~g} / \mathrm{l} \\ \mathrm{KCl} & 0.5 \mathrm{~g} / \mathrm{l} \\ \mathrm{CaCl}_{2} 2 \mathrm{H}_{2} \mathrm{O} & 0.18 \mathrm{~g} / \mathrm{l} \\ \mathrm{HEPES} & 2.4 \mathrm{~g} / \mathrm{l} \\ \mathrm{pH} \text { was adjusted to } 7.4\end{array}$

\subsection{Coating of plate with Collagen type I}

Collagen type I (Becton Dickinson) was diluted in $0.02 \mathrm{~N}$ acetic acid. The plates were coated with the concentration of $10 \mu \mathrm{g} / \mathrm{cm}^{2}$ and incubated for $1 \mathrm{~h}$ at $37^{\circ} \mathrm{C}$. Afterwards the rest of acetic acid was removed and the plates were washed twice with sterile $\mathrm{H}_{2} \mathrm{O}$. The plates were air-dried under clean bench and stored at $4^{\circ} \mathrm{C}$ until use. 


\subsection{In situ hybridization}

\subsubsection{Preparation of the riboprobes}

The riboprobes were prepared as digoxygenine and fluorescein labeled RNA. For single in situ hybridization both riboprobes (albumin and AFP) were labelled with digoxygenine. For double in situ hybridization albumin was labelled with fluorescein and AFP with digoxygenine.

\subsubsection{First-strand cDNA synthesis using M-MLV reverse transcriptase (RT)}

cDNA synthesis using a total RNA from E14 liver embryos was carried out as follows:

$1 \mu \mathrm{g}$ total RNA from E14 liver embryos dissolved in final volume of $10 \mu$ l sterile, distilled $\mathrm{H} 2 \mathrm{O}$.

Heat mixture to $65^{\circ} \mathrm{C}$ for 10 min and quick chill on ice

Collect the contents of the tube by brief centrifugation

Add $8 \mu \mathrm{l} 5 \times$ first strand buffer

$4 \mu \mathrm{l} 0.1 \mathrm{M}$ DTT

$8 \mu$ Oligo $(\mathrm{dT})_{12-18}(500 \mu \mathrm{g} / \mathrm{ml})$

$8 \mu 10$ mM dNTP Mix (10 mM each dATP, dGTP, dCTP and dTTP)

$2 \mu \mathrm{MMLV}$ RT

Mix contents of the tube by pipetting gently up and down and incubate at $37^{\circ} \mathrm{C}$ for $1 \mathrm{~h}$

Inactivate the reaction by heating at $90^{\circ} \mathrm{C}$ for $5 \mathrm{~min}$. 
The cDNA can now serve as template for amplification in PCR.

\subsubsection{Amplification of DNA by polymerase chain reaction (PCR)}

AFP and albumin sense and antisense probes were prepared by polymerase chain reaction (PCR) using Taq PCR Master Mix (Qiagen). The used primers are listed in table 1. When T7 promoter is upstream of the reverse primer, we obtain a cDNA construct for the synthesis of an antisense probe. When a T7 promoter is upstream of the forward primer, we generate a cDNA construct for the synthesis of sense probe

\section{PCR reaction:}

$25 \mu \mathrm{l}$ Taq PCR Master Mix

$5 \mu \mathrm{l}(5 \mu \mathrm{M})$ forward primer

$5 \mu \mathrm{l}(5 \mu \mathrm{M})$ reverse primer

$5 \mu \mathrm{l}$ cDNA

Volume was adjusted to $50 \mu$ with sterile $\mathrm{H}_{2} \mathrm{O}$

The PCR was carried out for 40 cycles under the following conditions:

$\begin{array}{lll}95^{\circ} \mathrm{C} & 10 \mathrm{~min} & \text { Denaturation step before the first cycle } \\ 95^{\circ} \mathrm{C} & 30 \mathrm{~second} & \text { Denaturation } \\ 65^{\circ} \mathrm{C} & 1 \mathrm{~min} & \text { Annealing } \\ 72^{\circ} \mathrm{C} & 1 \mathrm{~min} & \text { Extension } \\ 72^{\circ} \mathrm{C} & 10 \mathrm{~min} & \text { Final Extension }\end{array}$

\subsubsection{Agarose gel electrophoresis}

For preparation of a 1.5\% agarose gel, $1.25 \mathrm{~g}$ of agarose was dissolved by heating in $100 \mathrm{ml}$ of $1 \mathrm{X}$ TAE buffer. For visualization of the bands, $8 \mu \mathrm{l}$ of ethidium bromide $(10 \mathrm{mg} / \mathrm{ml})$ was added to the mixture. After mixing, 
the gel was poured into the prepared gel plate. The samples were prepared for loading by mixing $25 \mu \mathrm{l}$ of PCR product with $3 \mu \mathrm{l}$ of $6 \mathrm{X}$ loading dye solution (MBI Fermentas). After polymerization, the samples were loaded and the gel run was performed in $1 \times$ TAE buffer at $100 \mathrm{~V}$. The positive bands were excised and the amplified cDNA was purified by Gel Extraction Kit (Qiagen).

\section{$1 \times$ Tris/acetate/EDTA (TAE) buffer:}

Tris base

EDTA

$0.36 \mathrm{~g}$

Acetic acid $1.14 \mathrm{ml}$

Dissolved in 1 d distilled $\mathrm{H}_{2} \mathrm{O}$

$\mathrm{pH}$ was adjusted to 8.3 with acetic acid

\subsubsection{In vitro transcription}

The riboprobes albumin and AFP (sense and antisense) were labeled by using in vitro transcription reaction. This was set up at room temperature in the following order.

$1 \mu \mathrm{g}$ PCR product (DNA)

$2 \mu$ l Digoxygenine-U-dNTP or fluorescein-U-dNTP

$2 \mu \mathrm{l} 10 \times$ transcription buffer

$1 \mu \mathrm{l}$ RNase inhibitor

$2 \mu \mathrm{l} \mathrm{T7-RNA} \mathrm{polymerase} \mathrm{(5000} \mathrm{Units)}$

add $\mathrm{H}_{2} \mathrm{O}$ to make a final volume of $20 \mu \mathrm{l}$ and incubate the reaction at $37^{\circ} \mathrm{C}$ for $2 \mathrm{~h}$. Incubation with $2 \mu \mathrm{l}$ DNase I (10000 Units) at $37^{\circ} \mathrm{C}$ for $15 \mathrm{~min}$. The reaction was stopped with $2 \mu \mathrm{I} 0.2 \mathrm{M}$ EDTA. 


\subsubsection{Whole-mount in situ hybridization with digoxygenine-labelled AFP riboprobe}

Whole-mount in situ hybridization was essentially done as described previously (Harland, 1991) with modifications as reported by (Belo et al., 2000). 1 to 4 somites stages rat embryos (E10) were fixed immediately after dissection for overnight in $1 \times$ PBS to $\mathrm{pH} 7.4$ buffered $4 \%$ paraformaldehyde. Embryos were washed twice with PBSw and dehydrated through an increasing methanol gradient, and stored in $100 \%$ methanol at $-20^{\circ} \mathrm{C}$. Embryos were rehydrated through a reciprocal methanol gradient, and then washed three times for 5 min with PBSw on ice. Afterwards embryos were washed with $4.5 \mu \mathrm{g} / \mathrm{ml}$ proteinase $\mathrm{K}$ in PBSw for $30 \mathrm{~min}$. The digestion was stopped by washing the embryos in freshly prepared glycine solution, followed by two successive washes each 5 min in PBSw at room temperature. The embryos were refixed in $0.2 \%$ glutaraldehyde / $4 \%$ paraformaldehyde for $1 \mathrm{~h}$ at room temperature and rinsed three times in PBSw. A wash for about 5 min was performed with $50 \%$ PBSw / 50\% hybridization buffer, followed by $100 \%$ hybridization buffer. After $3 \mathrm{~h}$ prehybridization at $65^{\circ} \mathrm{C}$, the hybridization was carried out with antisense and sense riboprobes at $200 \mathrm{ng} / \mathrm{ml}$ in hybridization buffer overnight.

\subsubsection{Post-hybridization washes}

After overnight hybridization, the hybridization buffer was removed and the embryos were washed with $800 \mu \mathrm{l}$ of fresh hybridization buffer for $5 \mathrm{~min}$ at $70^{\circ} \mathrm{C}$. Afterwards, $400 \mu \mathrm{l}$ of $2 \times \mathrm{SSC}$ buffer, $\mathrm{pH} 4.5$ (without removing hybridization buffer) were added and the wash was repeated twice by adding $2 \times$ SSC buffer. The mix was removed and the embryos were further rinsed twice with $2 \times \mathrm{SSC}$ buffer, $\mathrm{pH} 7 / 0.1 \% \mathrm{CHAPS}$ at $70^{\circ} \mathrm{C}$ for 
30 min each time, followed by two successive Washes in maleic acid buffer, first for $10 \mathrm{~min}$ at RT and second for $30 \mathrm{~min}$ at $70^{\circ} \mathrm{C}$. A final wash was carried out in PBS for 10 min at RT and in PBSw for 5 min at RT.

\subsubsection{Blocking of embryos and antibody binding}

The embryos were incubated in $1 \mathrm{ml} 1 \mathrm{x}$ blocking solution for $2 \mathrm{~h}$ at $4^{\circ} \mathrm{C}$ with rocking. Afterwards, the embryos were incubated with antibody solution anti-digoxygenine overnight at $4^{\circ} \mathrm{C}$ with rocking.

\subsubsection{Post-antibody washes and signal detection}

After overnight incubation, the embryos were washed as follows:

1- $\quad$ Fast wash embryos with $0.1 \%$ BSA in PBSw

2- $\quad 5$ washes, 45 min each, with $5 \mathrm{ml} \mathrm{0.1 \%} \mathrm{BSA} \mathrm{in} \mathrm{PBSw}$

3- Wash twice, 30 min each in PBSw

4- Wash the embryos in detection buffer two times, 10 min each at RT with rocking.

5- Incubate the embryos in staining solution (NBT/BCIP) in the dark overnight at $4^{\circ} \mathrm{C}$ with slight rocking

6- $\quad$ Stop staining reaction by washing at least with three changes of PBS

7- After staining, dehydrate through methanol series and store in methanol at $-20^{\circ} \mathrm{C}$.

\subsubsection{Single and double in situ hybridization}

In situ hybridization experiments were performed according to a protocol described by (Harland, 1991) and (Pringle et al., 2003). The riboprobes were prepared as described previously. For single in situ hybridization both riboprobes (albumin and AFP) were labelled with digoxygenine. In 
case of double in situ hybridization, AFP was labelled with digoxigenine and albumin with fluorecein. $10 \mu \mathrm{m}$ sections of frozen embryos from developmental stages E12, E14, E16, E18 as well as new born and adult liver were fixed for $1 \mathrm{~h}$ with 4\% paraformaldehyde in PBS followed by two successive washes each 15 min with PBS treated with $0.1 \%$ active DEPC at RT. $100 \mu$ l hybridization buffer were given to the sections, covered, and then kept in humid chamber for prehybridization for $2 \mathrm{~h}$ at $65^{\circ} \mathrm{C}$. The hybridization was carried out overnight with labelled antisense and sense riboprobes of albumin and AFP at $500 \mathrm{ng} / \mathrm{ml}$ in hybridization buffer.

\subsubsection{Post-hybridization washes and signal detection}

After overnight hybridization the sections were washed in the following order:

1- Two successive washes at $60^{\circ} \mathrm{C}$, each $50 \mathrm{~min}$, first wash with $2 \mathrm{x}$ SSC and the second with $0.1 \times$ SSC.

2- $\quad$ RNase treatment was achieved with $10 \mu \mathrm{g} / \mathrm{ml}$ RNase A (Roche) in $2 \times \mathrm{SSC}$ buffer for $30 \mathrm{~min}$ at $37^{\circ} \mathrm{C}$,

3- $\quad$ A wash with $0.1 \times \mathrm{SSC}$ for $50 \mathrm{~min}$ at $60^{\circ} \mathrm{C}$.

4- Incubation of slides with blocking solution for $30 \mathrm{~min}$ at RT

5- $\quad$ For a single in situ hybridization, sections were incubated with alkaline phosphatase-conjugated anti-digoxygenine antibody for $2 \mathrm{~h}$ at RT

6- Wash slides twice, 15 min each, in washing buffer at RT

7- Incubate slides with detection buffer for $5 \mathrm{~min}$ at RT

8- Incubate the sections in staining solution (NBT/BCIP) in the dark overnight at RT

9- $\quad$ After staining, wash slides in distilled water for $10 \mathrm{~min}$ at RT

Slides were covered using a pre-warmed at $60^{\circ} \mathrm{C}$ liquid Kaiser gelatin. 


\subsubsection{Deactivation of the first alkaline phosphatase enzyme}

For double in situ hybridization, AFP mRNA positive slides (blue color) were further examined with alkaline-phosphate- conjugated antiFluorescein in an attempt to detect albumin expression. For this purpose alkaline phosphatase enzyme was deactivated by incubating the slides in washing buffer at $65^{\circ} \mathrm{C}$ for $30 \mathrm{~min}$. After two rinses with washing buffer at $\mathrm{RT}$, slides were treated with glycine-buffer $(0,1 \mathrm{M}$ glycine- $\mathrm{HCl} \mathrm{pH} 2,2)$ for 30 min followed by a wash in washing buffer at RT. Slides were now ready for alkaline phosphatase-conjugated anti-Fluorecein antibody. The incubation with antibody and the wash- steps were carried out as described previously. Signal detection was assessed using alkaline phosphatase-development solution (red colour) (Neubauer et al., 1996). The slides were incubated overnight in alkaline phosphatase-development solution in the dark at room temperature followed by washing in distilled water for 10 min at RT.

\subsubsection{In situ hybridization in explanted cells}

In situ hybridization was also performed in 8-chambered lab-tecks after two days in cultue of hepatocyte, hepatoblasts and endodermal cells generated from ventral foregut endoderm. Fixation with $4 \%$ paraformaldehyde, prehybridization, hybridization and signal detection were performed as described previously.

\subsubsection{Solutions and buffers}

PBS:

PBS was mixed with $0.1 \%(\mathrm{v} / \mathrm{v})$ DEPC, incubated overnight and then sterile autoclaved. Before use, PBS was retreated with fresh $0.1 \%$ DEPC. 
PBSw:

PBS with $0.1 \%(v / v)$ Tween-20

\section{Glycine solution:}

$0.2 \%(w / v)$ glycine was dissolved in PBSw

\section{4\% Paraformaldehyde:}

$4 \mathrm{~g}$ Paraformaldehyde were dissolved in $100 \mathrm{ml}$ RNase free PBS under basic condition $(500 \mu \mathrm{l} \mathrm{NaOH})$ at $60{ }^{\circ} \mathrm{C}$. $\mathrm{pH}$ was adjusted to 7.4

20x SSC Buffer:

$3 \mathrm{M} \mathrm{NaCl}$

$0.3 \mathrm{M}$ Tri-sodium citrate

dissolved in $\mathrm{H}_{2} \mathrm{O}$ (treated with 0.1\% DEPC), $\mathrm{pH} 7$

\section{Deionized formamide:}

$500 \mathrm{ml}$ Formamide was mixed with $50 \mathrm{~g}$ mixed Bed Resin. The mixture was stirred for $30 \mathrm{~min}$ in the dark at room temperature. Afterwards, it was sterile filtered, dispensed into $50 \mathrm{ml}$ aliquots and stored in the dark at $20^{\circ} \mathrm{C}$.

Hybridization buffer:

50\% (v/v) deionized Formamide

$4 \times$ SSC Buffer

10\% (v/v) Herings-Sperm 10 mg/ml

$10 \mathrm{mM}$ dithiothreitol 
$0.05 \%(w / v)$ tRNA yeast

$0.1 \%(\mathrm{w} / \mathrm{v})$ dextran sulfate

dissolved in $\mathrm{H}_{2} \mathrm{O}$ treated with $0.1 \%(\mathrm{v} / \mathrm{v})$ DEPC

Maleic acid:

0.1 M Maleic acid

$0.15 \mathrm{M} \mathrm{NaCl}$

dissolved in $1 \mathrm{l} \mathrm{H}_{2} \mathrm{O}$

$\mathrm{pH}$ was adjusted to 7.5 by $\mathrm{NaOH}$ pellets.

Washing buffer:

$0.3 \%(\mathrm{v} / \mathrm{v})$ Tween dissolved in maleic acid.

10 x Blocking solution:

$5 \mathrm{~g}$ Blocking reagent were dissolved in $50 \mathrm{ml}$ maleic acid by stirring and heating at $65^{\circ} \mathrm{C} .10 \times$ blocking solution stock was autoclaved and stored at $-20^{\circ} \mathrm{C}$.

\section{Antibodies solution:}

The antibodies anti-digoxygenin-alkaline phosphatase conjugated (AntiDIG-AP) and anti-fluorescein-alkaline phosphatase conjugated (Anti-FLAP) were centrifuged for $5 \mathrm{~min}$, at $10000 \mathrm{rpm}$. The supernatants were diluted to a concentration of 1:500 in 1x blocking solution. 


\section{Detection buffer:}

$100 \mathrm{mM}$ tris- $\mathrm{HCl}$

$100 \mathrm{mM} \mathrm{NaCl}$

dissolved in $\mathrm{H}_{2} \mathrm{O}$, pH 9.5

0.024\% (w/v) levamisol was added fresh before use.

\section{Staining solution:}

$250 \mu \mathrm{l}$ nitroblue tetrazolium salt (NBT) and $188 \mu \mathrm{l}$ 5-bromo-4-chloro-3indolyl-phosphate (BCIP) were dissolved in $60 \mathrm{ml}$ detection buffer with Levamisol. For double in situ hybridization, alkaline-phosphatasedevelopment solution was also used (Neubauer et al., 1996).

\section{Alkaline phosphatase-development solution:}

\section{Solution A:}

- $70 \mathrm{ml}$ development buffer containing:

$4.9 \mathrm{~g}$ Tris-Base

$8.7 \mathrm{~g} \mathrm{NaCl}$

$1.5 \mathrm{~g}$ Tris- $\mathrm{HCl}$

$\mathrm{pH}$ was adjusted to 9.7 with $1 \mathrm{~N} \mathrm{HCl}$

Fill to $1 \mathrm{I}$ distilled $\mathrm{H}_{2} \mathrm{O}$

- $25 \mathrm{ml}$ propandiol

- 40 mg levamisol

\section{Solution B:}

50 mg Naphthol-AS-BI-phosphate disodium salt (Na-As-Bi-P) dissolved in $0.6 \mathrm{ml} \mathrm{N}$-N-Dimethylformamide (DMF). 


\section{Solution C:}

$20 \mathrm{mg}$ sodiumnitrite $\left(\mathrm{NaNO}_{2}\right)$ dissolved in $0.5 \mathrm{ml} \mathrm{H}$ O. Afterwards $0.2 \mathrm{ml}$ Neufuchsin was added.

Alkaline phosphatase-development solution was freshly prepared as follows:

Solution $\mathrm{C}$ was mixed with solution $\mathrm{A}$ before adding solution $\mathrm{B}$. $\mathrm{pH}$ was adjusted to 8.8 with $2 \mathrm{~N} \mathrm{HCl}$. The mixture was filtered bfore use.

\subsection{Proliferation assay using PCNA immunostaining}

\subsubsection{PCNA-staining by immunoperoxidase technique}

PCNA immunohistochemistry experiment was performed by immunoperoxidase technique to detect proliferating cell nuclear antigen (PCNA) (Micsenyi et al., 2004). $5 \mu \mathrm{m}$ cryosection of rat embryos from developmental stage E12, E14, E16, E18 as well as new born and adult liver were fixed with $4 \%$ paraformaldehyde for 30 min at RT followed by an incubation step in $70 \%$ ehtanol at $-20^{\circ} \mathrm{C}$. Subsequently the slides were rinsed twice in phosphate-buffered saline (PBS). Slides were microwaved for $40 \mathrm{~min}$ in citrate buffer at $\mathrm{pH} 6$ followed by incubation for $10 \mathrm{~min}$ in Triton buffer. Endogenous peroxide was inactivated after incubation in glucose solution for $30 \mathrm{~min}$ at $37^{\circ} \mathrm{C}$. After two successive washes with PBS, sections were blocked with FCS for $20 \mathrm{~min}$, washed again and incubated overnight at $4^{\circ} \mathrm{C}$ in PBS diluted (1:100) mouse anti-proliferating cell nuclear antigen (anti-PCNA) antibody. The next day, sections were washed and incubated with the secondary anti-mouse horseradish peroxidase (HRP)-conjugated antibody (1:100) for $1 \mathrm{~h}$ at RT. A signal was detected using diaminobenzidine $/ \mathrm{H}_{2} \mathrm{O}_{2}(0.05 \%(\mathrm{w} / \mathrm{v}) / 0,01 \%(\mathrm{v} / \mathrm{v}))$. The sections were counter-stained with hemalaun. After several washes with $\mathrm{H}_{2} \mathrm{O}$, the slides were covered using a pre-warmed at $60^{\circ} \mathrm{C}$ liquid Kaiser gelatin 


\subsubsection{Buffers}

\section{Citrate buffer:}

$1.92 \mathrm{~g}$ citric acid -monohydrate dissolved in $1 \mathrm{l} \mathrm{H}_{2} \mathrm{O}$

$\mathrm{pH}$ was adjusted to 6 with $2 \mathrm{~N} \mathrm{NaOH}$

\section{Triton buffer:}

$0.3 \%(v / v)$ Triton

$0.03 \%(v / v) \mathrm{H}_{2} \mathrm{O}_{2}$

Dissolved in PBS

\section{Glucose solution:}

$0.1 \mathrm{mM}$ Glucose

0.19 units Glucoseoxidase

1 M Sodium azide

Dissolved in PBS

\subsection{Quantitative morphological studies}

Quantitation of in situ hybridization of albumin and AFP and of PCNA immunohistochemical reactions were performed by counting of positive cells under microscope using a shaded ocular, or by application of Image J software (Wayne Rasband, NIH, USA), and relating the positive cells to the whole cell counts of the analyzed image area.

\subsection{RNA extraction}

\subsubsection{Isolation of RNA by density-gradient ultracentrifugation}

Total RNA was isolated from whole liver (in vivo) and cultured hepatoblats and hepatocyte (in vitro) at different development satge as well as from 
new born and auldt liver by means of guanidine isothiocyanate extraction, cesium chloride density-gradient ultracentrifugation and ethanol precipitation according to method of Chirgwin (Chirgwin et al., 1979) as described elsewhere (Ramadori et al., 1985). Frozen tissues as well as cells were homogenized in ice-cold GITC buffer. $2 \mathrm{ml}$ of $\mathrm{CsCl}_{2}$ buffer was poured into $5 \mathrm{ml}$ polyallomer ultracentrifuge tubes (6 per preparation). The cleared guanidine lysed samples were carefully layered on top of the $\mathrm{CsCl}_{2}$ buffer. The samples were centrifuged overnight (16 h) at 35,000 $\mathrm{rpm}$ in a Kontron TST55 rotor at $20^{\circ} \mathrm{C}$. The supernatants were carefully removed by aspiration and the transparent RNA pellets were gently washed twice with $200 \mu \mathrm{l}$ of $70 \%$ ethanol at room temperature. The pellets were reconstituted in $200 \mu \mathrm{l}$ of RNase-free water by pipetting and transferred into sterile $1.5 \mathrm{ml}$ reaction tubes. The procedure was immediately continued by RNA precipitation. The RNA was precipitated with $400 \mu \mathrm{l}$ of $100 \%$ ethanol in the presence of $20 \mu \mathrm{l}$ sodium-acetate solution overnight at $-20^{\circ} \mathrm{C}$. The RNA precipitates were pelleted by centrifugation for $30 \mathrm{~min}$ at $12,000 \mathrm{rpm}$ and $4^{\circ} \mathrm{C}$. Supernatants were discarded and pellets were washed with $200 \mu \mathrm{l}$ of ice-cold $70 \%$ ethanol to remove all traces of sodium-acetate. After subsequent recentrifugation as described, the supernatants were discarded and the pellets were air dried. Afterwards, the pellets were dissolved in $100 \mu \mathrm{l}$ of RNase-free water. To determine the concentration and purity of the obtained RNA, the aliquot of RNA sample was diluted 1:100 in RNase-free $\mathrm{H}_{2} \mathrm{O}$ and the extinction at $260 \mathrm{~nm}$ and $280 \mathrm{~nm}$ was measured spectrophotometrically (GeneQuant II, Pharmacia Biotech).

\subsubsection{Buffers}

\subsection{M sodium citrate:}

7.36\% (w/v) sodium citrate solution was prepared in RNase Free $\mathrm{H}_{2} \mathrm{O}$ $\mathrm{pH}$ was adjusted to 7 with $0.25 \mathrm{M}$ citric acid 
(Guanidine isothiocyanate) GITC buffer:

Guanidine isothiocyanate

$0.25 \mathrm{M}$ sodium citrate

$\mathrm{N}$-laurylsarcosyl

$0.5 \%(\mathrm{w} / \mathrm{v})$

Dissolved in RNase-free $\mathrm{H}_{2} \mathrm{O}$

The solution was sterile filtered and stored in the dark at $4^{\circ} \mathrm{C}$. $0.1 \mathrm{M} \beta$ Mercaptoethanol was added just before use.

\section{CsCl buffer:}

Cesium chloride

Sodium citrate

$0.25 \mathrm{M}$

EDTA

$0.5 \mathrm{M}$

Dissolved in RNase-free $\mathrm{H}_{2} \mathrm{O}$.

$\mathrm{pH}$ was adjusted with $0.25 \mathrm{M}$ citric acid to 7.5 ; the solution was sterile filtered and stored at room temperature.

\section{$2 \mathrm{M}$ Sodium-acetate solution:}

$16.4 \%$ (w/v) Sodium-acetat dissolved in Rnase-free $\mathrm{H}_{2} \mathrm{O}$ $\mathrm{pH}$ was adjusted to 5.4 with acetic acid

\subsection{Real-time $P C R$ analysis}

For the quantitative analysis of albumin, AFP, HNF4alpha, beta-catenin, Prox1, Foxa-2, GATA-4, BMP-4, Interleukin-6 receptor (IL-6rec), granulocyte-macrophage colony-stimulating factor (GM-CSF), granulocyte colony-stimulating factor (G-CSF), stem cell factor (SCF) and Erythropoietin (Epo) specific transcripts, real-time RT-PCR was performed. Total RNA was extracted from cultured hepatoblasts (in vitro) and whole liver (in vivo) at different development stages. Their cDNA was obtained by using M-MLV Reverse Transcriptase (RT) Kit as described 
previously. cDNA from explanted endodermal cells derived from ventral foregut and hepatoblasts at E12 was prepared by Fastlane Cell cDNA kit (Qiagen). Realtime PCR experiments were performed using an ABI Prism 7000 thermal cycler (Qiagen), SYBRß Green PCR Mastermix (invitrogen), cDNA and specific primers (Table 2). The gene expression levels of target genes (Q) was determined based on the threshold PCR cycle-values ( $\left.\mathrm{Ct}_{\text {target}}\right)$ (following the instructions by AppliedBiosystems) and it was normalized with the threshold PCR cycle-values of the endogenous control of ribosomal RNA $18 \mathrm{~S}\left(\mathrm{Ct}_{18 \mathrm{~S}}\right)$, using the following formula: $\mathrm{Q}=2^{-\Delta \mathrm{Ct}}$, where $\Delta \mathrm{Ct}=\mathrm{Ct}_{\text {target }}-\mathrm{Ct}_{18 \mathrm{~S}}$.

\subsection{Fluorescent immunostaining}

Immunofluorescence study was performed in $5 \mu \mathrm{m}$ cryos-ections of rat embryos and livers removed from newborn and adult rats as well as endodermal cells generated from ventral foregut (E10) and hepatoblasts from developmental stage E12, E14, E18, cultured for two days on 8chambered lab-Tecks were fixed in acetone for $10 \mathrm{~min}$ at room temperature and rinsed in phosphate-buffered saline. Blocking of nonspecific binding with $1 \%(\mathrm{w} / \mathrm{v})$ bovine serum albumin and 10\% (v/v) goat serum (DAKO, Glostrup, Denmark) in PBS was carried out for $1 \mathrm{~h}$ at RT. Endodermal cells generated from ventral foregut and hepatoblasts from E12 were immunostained by rabbit polyclonal antibody against HNF4alpha (1:50) overnight at $4^{\circ} \mathrm{C}$. In cryosection of embryos and hepatoblasts from different developmental stages a double immunostainings was performed. The first reaction was done overnight at $4^{\circ} \mathrm{C}$ with rabbit polyclonal anti-Prox 1 antibody diluted to $1: 500$, rabbit polyclonal anti-AFP antibody diluted to $1: 100$, mouse monoclonal antibodies anti-cytokeratin (CK) 7 (1:50), anti-connexin 32 (1:100), antiCK19 (1:100) and HepPar1 (1:50). Rabbit polyclonal antibodies were 
detected with Alexa 555-conjugated secondary anti-rabbit antibodies 1:400 diluted in PBS. Mouse monoclonal antibodies were visualized with Alexa 488-conjugated secondary anti-mouse antibodies 1:200 diluted in PBS. Sections and lab-Tecks were counter-stained with DAPI, covered using Fluoromount and analysed with epifluorescence microscopes (Axioskop 50 or Axiovert 200M, with Apotome function) (Zeiss, Jena, Germany).

\subsection{Quantitative analysis of Prox1-, CK19- and AFP-specific immunohistochemical reactions in embryonal and foetal rat livers}

The sections of embryonal (E14) and foetal livers (E18) were stained simultaneously with monoclonal anti-CK-19 antibody and with polyclonal anti-Prox1 or anti-AFP antibodies. The antibodies were visualised as described above, CK-19 was detected in green, while Prox1 or AFP in red, and all cell nuclei were counter-stained with DAPI in blue. Images of red, green and blue channels were taken by an Axiovert 200M (Zeiss, Jena, Germany) microscope with the Apotome function, equipped by a high resolution Axiocam camera. Double positive cells were determined by images of combined red green channels. All single channels and red green combined channels were analysed, and positive cells were counted by the ImageJ software (Wayne Ras-band, NIH, USA). Single and double positive cell counts were related to all cell nuclei in the image (determined by DAPI staining), and expressed in average $\%+$ standard error of measurement (SEM) \%. In all quantitative immunohistochemical analysis 15 random taken samples from the investigated sections were analysed. 
2.17. Radioactive biosynthetic labelling, immunoprecipitation and SDS-PAGE analysis

\subsubsection{Radioactive biosynthetic labeling with ${ }^{35}-\mathrm{S}$ Methionine}

Newly synthesized proteins were radioactively labelled with ${ }^{35} \mathrm{~S}$ methionine as described before (Ramadori et al., 1990). Briefly, endodermal cells derived from venral foregut, hepatoblats at different development stages and hepatocytes were cultured in DIF medium for 2 days in 24 well-plate. After this, cells were washed 3 times with methionine-free RPMI medium and incubated in RPMI medium supplemented with ${ }^{35} \mathrm{~S}$-methionine $(100 \mu \mathrm{Ci} /$ well $)$ for $2 \mathrm{~h}$. Cells derived from venral foregut were incubated overnight.

\subsubsection{Preparation of cell-lysates and measurement of total labeled proteins after trichloracetic acid precipitation.}

After labelling, supernatants were harvested and diluted to $50 \%$ with lysis mix with SDS (LM+SDS). The cells were lysed after freeze-thawing and scraping in lysis mix without sodium dodecylsulfate (SDS) (LM-SDS) suplemented with $1 \%(\mathrm{v} / \mathrm{v})$ phenylmethylsulfonyl fluoride (2 mM PMSF in ethanol, pH 7.4). Cell-lysates were then harvested and diluted to $50 \%$ with lysis mix with SDS (LM+SDS). Supernatants and cell-lysates were stored till use at $-80^{\circ} \mathrm{C}$. The count of the total labelled proteins was measured after a trichloroacetic acid precipitation as follows:

1- $5 \mu \mathrm{l}$ of cell-lysates and supernatants from each sample were transferred to a small piece of whatman paper

2- $\quad$ The whatman papers were air-dried 
3- Incubated in 10\% (w/v) trichloracetic acid (10\% TCA supplemented with $0.75 \%(w / v)$ L-methionine) for $10 \mathrm{~min}$ at RT

4- Incubated in pre-warmed $5 \%(\mathrm{w} / \mathrm{v})$ TCA supplemented with $1.5 \%$ $(\mathrm{w} / \mathrm{v})$ L-methionine for $15 \mathrm{~min}$ at $80^{\circ} \mathrm{C}$

5- $\quad$ Two times wash with $5 \%(\mathrm{w} / \mathrm{v})$ TCA supplemented with $1.5 \%(\mathrm{w} / \mathrm{v})$ L-methionine at RT

6- Wash with $96 \%$ ethanol at RT

7- $\quad$ Wash with ethanol/acetone mix 1:1 at RT

8- Wash with $100 \%$ acetone at RT

9- The wathman papers were air-dried.

10- The wathman papers were transferred to scintillation tubes and then filled with scintillation liquid ( $5 \mathrm{ml}$ per tube)

11- Measurement of Beta radioactivity

The measured radioactivity correspond to total labelled proteins, which incorporate ${ }^{35} \mathrm{~S}$ - methionine.

2.17.3. Immunoprecipitation of albumin and AFP by polyclonal antialbumin and anti-AFP antibodies.

\subsubsection{Preparation of protein A}

Protein A was prepared in lysis mix with SDS supplemented with $0.5 \%$ BSA. $1 \times$ volume protein A was washed twice with lysis mix with SDS (LM+SDS) supplemented with $0.5 \%$ BSA and then resuspended in the original volume. Protein $A$ is now ready for use. 


\subsubsection{Immunoprecipitation}

For immunoprecipitation of albumin and AFP, $50 \mu \mathrm{l}$ of supernatants and cell-lysates (with the same count of total labeled proteins precipitated by trichloroacetic acid) from labeled hepatocyte and hepatoblasts at different developmental stages were used. From labelled cells generated from ventral foregut endoderm, $500 \mu \mathrm{l}$ of supernatants and cell-lysates were taken for analysis. The samples were incubated first with $50 \mu$ protein A for 30 min on ice. After centrifugation, the pellet was discarded and precleared samples were incubated with a monospecific rabbit polyclonal anti-albumin and anti-AFP overnignt at $4^{\circ} \mathrm{C}$. Immunocomplexes were precipitated by adding $50 \mu \mathrm{l}$ of protein $\mathrm{A}$ and incubation for $1 \mathrm{~h}$ on ice. Immunoprecipitates were sedimented, washed first once with lysis mix with SDS, suplemented with $0.5 \%$ BSA followed by 4 washes with lysis mix with SDS and finally resuspended in $25 \mu$ loading buffer suplemented with fresh $\beta$-mercaptoethanol. The samples were stored for $5 \mathrm{~min}$ or overnight at $-80^{\circ} \mathrm{C}$.

\subsubsection{SDS-polyacrylamide gel electrophoresis (SDS-PAGE) analysis}

Immunoprecipitated albumin and AFP were analysed by $12 \%$ SDSpolyacrylamide gel electrophoresis (SDS-PAGE) according to (Laemmli, et al., 1970). The samples were thawed, resuspended after long time vortexing and boiled for $5 \mathrm{~min}$ at $90^{\circ} \mathrm{C}$. After high speed centrifugation for 15 min, pellet was discarded and supernatants were loaded on gel. The electrophoresis was performed at $200 \mathrm{~V}$ for $4 \mathrm{~h}$. Afterwards the gels were fixed overnight with fixation solution, incubated for $1 \mathrm{~h}$ in Amplify $^{\mathrm{TM}}$ Fluorographic Reagent, covered with Cell-glas (Cellophane) and then 
dried using Gel Dryer System (Biorad). Dried gels were placed in X-ray film cassette and exposed to X-ray film.

\section{Solution A:}

18.2\% (w/v) Tris-base dissolved in distilled $\mathrm{H}_{2} \mathrm{O}, \mathrm{pH} 8.8$

\section{Solution B:}

$30 \%(\mathrm{w} / \mathrm{v})$ acrylamide and $0.8 \%(\mathrm{w} / \mathrm{v})$ Bis-acrylamide dissolved in distilled $\mathrm{H}_{2} \mathrm{O}$, stored in the dark at $4^{\circ} \mathrm{C}$

\section{Solution C:}

$7.8 \%(\mathrm{w} / \mathrm{v})$ Tris- $\mathrm{HCl}, \mathrm{pH} 6.8$

$20 \%(w / v)$ SDS

$40 \%(w / v)$ ammonium persulfate (APS)

Running buffer:

3.028 g Tris-Base

14.4 g Glycine

$5 \mathrm{ml}$ of $20 \%$ SDS

fill to $1 \mathrm{I}$ distilled $\mathrm{H}_{2} \mathrm{O}$

\section{Fixation solution:}

$460 \mathrm{ml}$ methanol

$80 \mathrm{ml}$ acetic acid

$460 \mathrm{ml}$ distilled $\mathrm{H}_{2} \mathrm{O}$ 


\begin{tabular}{|c|c|c|}
\hline SDS-PAGE & $\begin{array}{c}\text { Running gel } \\
(12 \%)\end{array}$ & $\begin{array}{c}\text { Stacking gel } \\
(3 \%)\end{array}$ \\
\hline solution A & $18 \mathrm{ml}$ \\
solution B & $28.4 \mathrm{ml}$ & $2.7 \mathrm{ml}$ \\
solution C & $\mathrm{X}$ & $2 \mathrm{ml}$ \\
distilled $\mathrm{H}_{2} \mathrm{O}$ & $24.6 \mathrm{ml}$ & $15.2 \mathrm{ml}$ \\
$20 \%$ SDS & $360 \mu \mathrm{l}$ & $100 \mu \mathrm{l}$ \\
TEMED & $20 \mu \mathrm{l}$ & $20 \mu \mathrm{l}$ \\
40\%APS & $60 \mu \mathrm{l}$ & $20 \mu \mathrm{l}$ \\
\hline
\end{tabular}

\subsubsection{Reagents and Buffers:}

Radioactive methionine: ${ }^{35} \mathrm{~S}-$ methionine

RPMI medium without methionine:

supplemented with $100 \mathrm{U} / \mathrm{ml}$ penicillin, $100 \mu \mathrm{g} / \mathrm{ml}$ streptomycin, 1\% Lglutamine, $0.1 \mu \mathrm{g} / \mathrm{ml}$ insulin, $0.1 \mu \mathrm{g} / \mathrm{ml}$ glucagon and $0.1 \mu \mathrm{g} / \mathrm{ml}$ dexamethasone.

Lysis mix without sodium-n-dodecyl sulfate (SDS) (LM-SDS):

0.5\% (w/v) deoxycholic acid (DOC)

1\% Triton $\mathrm{x}-100$

10 mM ethylene-diaminetetraacetic acid (EDTA) 
Dissolved in $500 \mathrm{ml} \mathrm{PBS.} \mathrm{pH}$ was adjusted to 7.4

\section{Lysis mix with SDS (LM+SDS) :}

$5 \mathrm{~g}$ SDS was added to LM-SDS

\section{PMSF(phenylmethysulfonylfluoride):}

$35.5 \mathrm{mg}$ PMSF dissolved in $1 \mathrm{ml}$ ethanol; before use $10 \mu \mathrm{l}$ was added to 1 $\mathrm{ml}$ lysis mix without SDS.

Protein A (sigma):

\section{LM+BSA:}

$0.5 \%(w / v)$ BSA were dissolved in lysis mix with SDS.

\section{Loading Buffer:}

$12.5 \mathrm{ml}$ of $20 \%(\mathrm{w} / \mathrm{v})$ SDS

157 mg TRIS-Base,

$21 \mathrm{ml}$ glycerine,

Bromophenolblue,

add $100 \mathrm{ml} \mathrm{H}$ O, pH was adjusted to 6.8.

Before use, $950 \mu$ l loading Buffer was supplemented with $50 \mu \mathrm{l} \beta$ -

mercaptoethanol.

trichloroacetic acid:

10\% TCA: 10\% (w/v) trichloroacetic acid and 0.75\%(w/v) Methionine dissolved in $\mathrm{H}_{2} \mathrm{O}$ 
5\% TCA: 5\% (w/v) trichloracetic acid and 0.15\% (w/v) Methionine dissolved in $\mathrm{H}_{2} \mathrm{O}$

\section{Ethanol and acetone:}

$96 \%$ ethanol

$100 \%$ Acetone

Ethanol/acetone (1:1)

\subsection{8. ${ }^{35}$ Smethionine pulse-chase labelling}

A pulse chase experiment was carried out according to the protocol described by (Tworkowski et al., 2002). Hepatoblasts and hepatocyte from different developmental stages E12, E14, E16, E18, and adult were cultured in DIF medium for two days in 24 well-plate. The cells were washed three times with methionine free RPMI medium and then pulsed for $1 \mathrm{~h}$ in RPMI medium suplemented with $100 \mu \mathrm{Ci} /$ well ${ }^{35} \mathrm{~S}$-methionine. Subsequently three washes with PBS were performed to remove traces of radioactive methionine. The cells were further cultured in radioactive free RPMI medium suplemented with $2 \mathrm{mM}$ L-methionine and incubated during a chase period of $15,30,45,60,90,120,240 \mathrm{~min}$. At each period of chase, cell-lysates and supernatants were collected, diluted to $50 \%$ with lysis mix with SDS (LM+SDS) and stored till use at $-80^{\circ} \mathrm{C}$. Albumin and AFP were immunoprecipitated and analysed as described previously.

Pulse chase analysis was further performed in hepatoblasts at 12, 14, 16 and 18 days of gestation after 2 and 9 days in culture. The hepatoblasts were pulsed for $1 \mathrm{~h}$ with ${ }^{35} \mathrm{~S}$ methionine and incubated in radioactive free RPMI medium supplemented with $2 \mathrm{mM}$ methionine during a chase period of 2, 4 and $8 \mathrm{~h}$. Albumin immunoprecipitation was performed as described previously. In this case immunocomplexes were not analysed by SDS gel electrophoresis (SDS-PAGE), synthesis and secretion of albumin was 
analysed by measuring the radioactivity of albumin immunocomplexes using a beta counter and the ratio of count of albumin immunocomplexes to the count of total labelled protein was diagramed at each chase period.

\subsection{Interleukin-6 (IL6) treatment}

For Interleukin-6 (IL6) treatment, hepatoblasts from developmental stages E12, E14 and E18 were used. After two days in culture in 24 well-plate, the cells were treated simultaneously with $100 \mathrm{ng} / \mathrm{ml}$ interleukin-6 (IL6) and radioactive labelled with $100 \mu \mathrm{ci}{ }^{35} \mathrm{~S}$-methionine per well overnight. Cell-lysates and supernatants were collected. Synthesis and secretion of albumin and AFP was examined by immunoprecipitation of albumin and AFP combined with SDS-PAGE as described previously. Interleukin-6 receptor (IL6rec) mRNA expression in untreated hepatoblasts from E12, E14 and E18 was measured by real-time PCR. RNA was extracted from cultured hepatoblasts, cDNA was obtained by using M-MLV Reverse Transcriptase (RT) Kit and realtime PCR experiment was performed as described previously. Primers for Interleukin-6 receptor (IL6rec) are listed in table 2. GAPDH was used as housekeeping gene.

\subsection{Analysis of albumin and AFP gene expression in hepatoblasts after passage.}

Hepatoblasts isolated at developmental stages E12 and E14 were kept in culture and passaged for three times. At each passage, the cells were cultured in 24 well-plate and labeled with $100 \mu \mathrm{ci}^{35} \mathrm{~S}$-methionine per well. Cell-lysates and supernatants were analysed by measurement of synthesis and secretion of albumin and AFP. Immunoprecipitation of albumin and AFP combined with SDS-PAGE were performed as described previously. At each passage $100 \mu \mathrm{l}$ supernatant and cell-lysates were used for immunoprecipitation of albumin and AFP. As positive control $25 \mu \mathrm{l}$ supernatants and cell-lysates of labelled hepatocyte were 
used for albumin precipitation. Furthermore, expression of albumin and AFP mRNA at each passage was analysed by real-time PCR. At each passage RNA was extracted and cDNA was prepared by using M-MLV reverse transcriptase (RT) Kit. Real-time PCR experiment was performed as described previously. Primers for albumin, AFP are listed in table 2. GAPDH was used as housekeeping gene.

\subsection{Statistical analysis}

The data were analysed using Prism Graph pad 4 software (San Diego, USA) and Microsoft Office Exel 2003. All experimental errors are shown as S.E.M. Statistical significance was calculated by ANOVA and student's $t$-test. Significance was accepted at $P<0.05$. 


\section{RESULTS}

\subsection{Morphological observations}

Endodermal cells were isolated from ventral foregut region at 10 days of gestation. Detailed morphological and functional analysis of endodermal cells has not yet been performed so far. Therefore, in the current work studying morphological features of endodermal cells preceded subsequent experiments.

It was observed that endodermal cells were composed of cells which displayed different morphological features. After six months in culture, three cell-populations could be identified. First cell-population (cellpopulation I) consist of cells with polyhedric morphology. Some cells of them are diploid. Second cell-population (cell-population II) consists of cells with round structure and some cells displayed double-nuclei. Third cell-population (Cell-population III) consists of cells, which show fibroblastlike morphology with spindle-shaped appearance (Fig. 4).

The hepatoblasts were isolated from embryonic/foetal liver at developmental stages E12, E14, E16, E18, E20 and new born liver and then cultured on coated plate for two days. The hepatoblasts grew as colony surrounded by fibroblasts-like cells. We noticed that the volume of hepatoblasts increases and the number of chromosomes in hepatoblasts becomes doubled (polyploidy) during liver development (Fig. 5). 


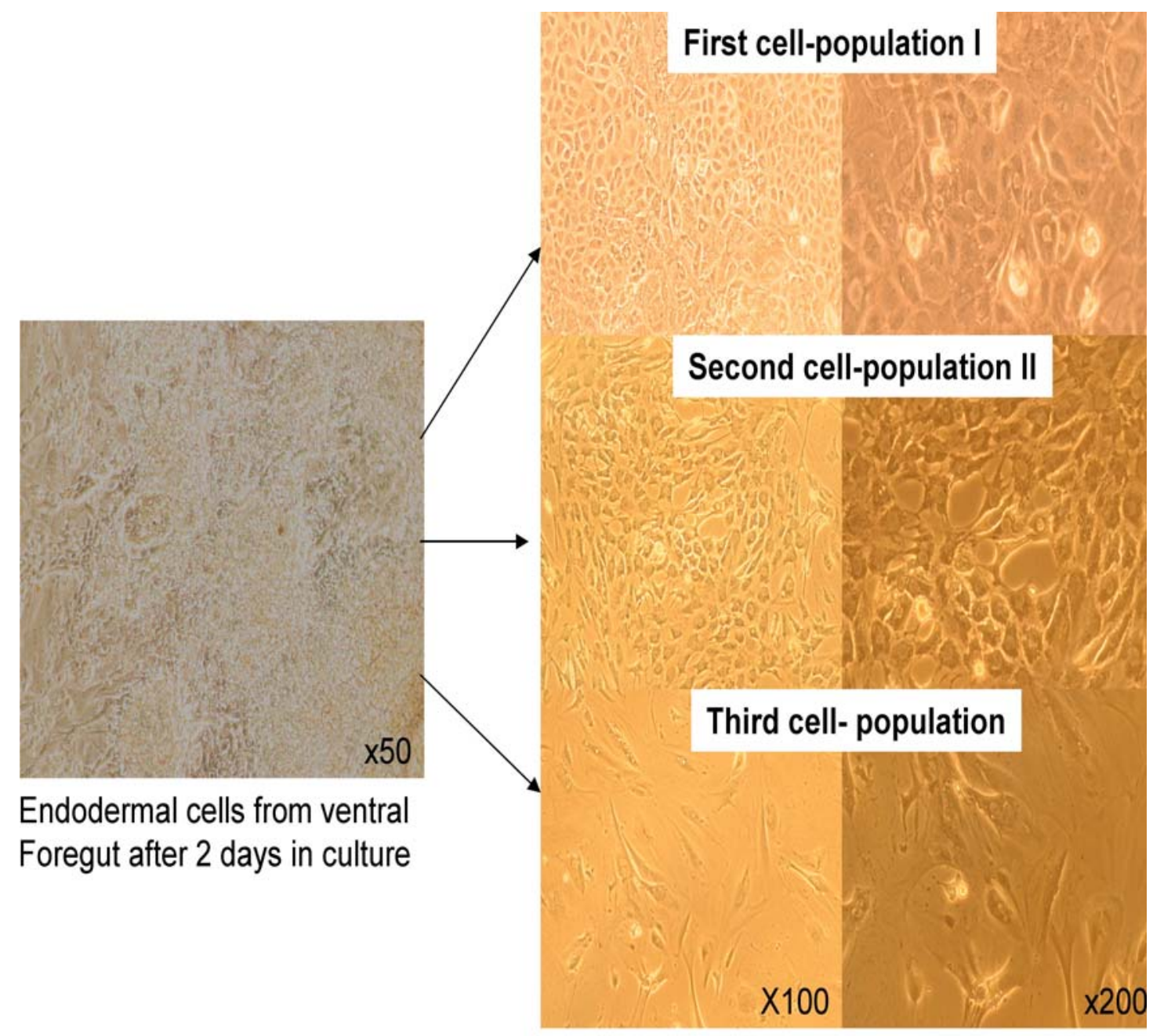

Endodermal cells from ventral foregut after six months in culture

Figure 4: Morphological and cultural features of endodermal cells derived from ventral foregut region. Three cell-population were identified after six months in culture as assessed by phase-contrast microscopy. Original magnification x 50; $x$ $100 ; \times 200$. 

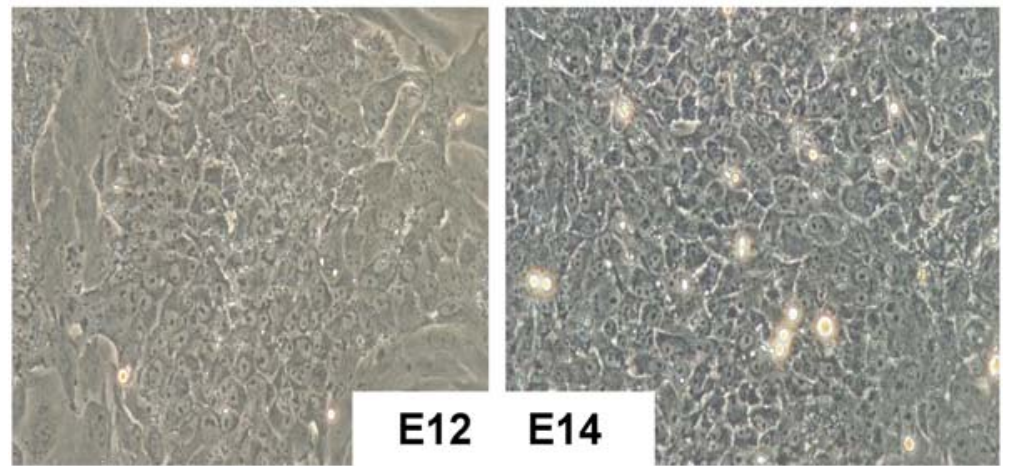

E12 E14
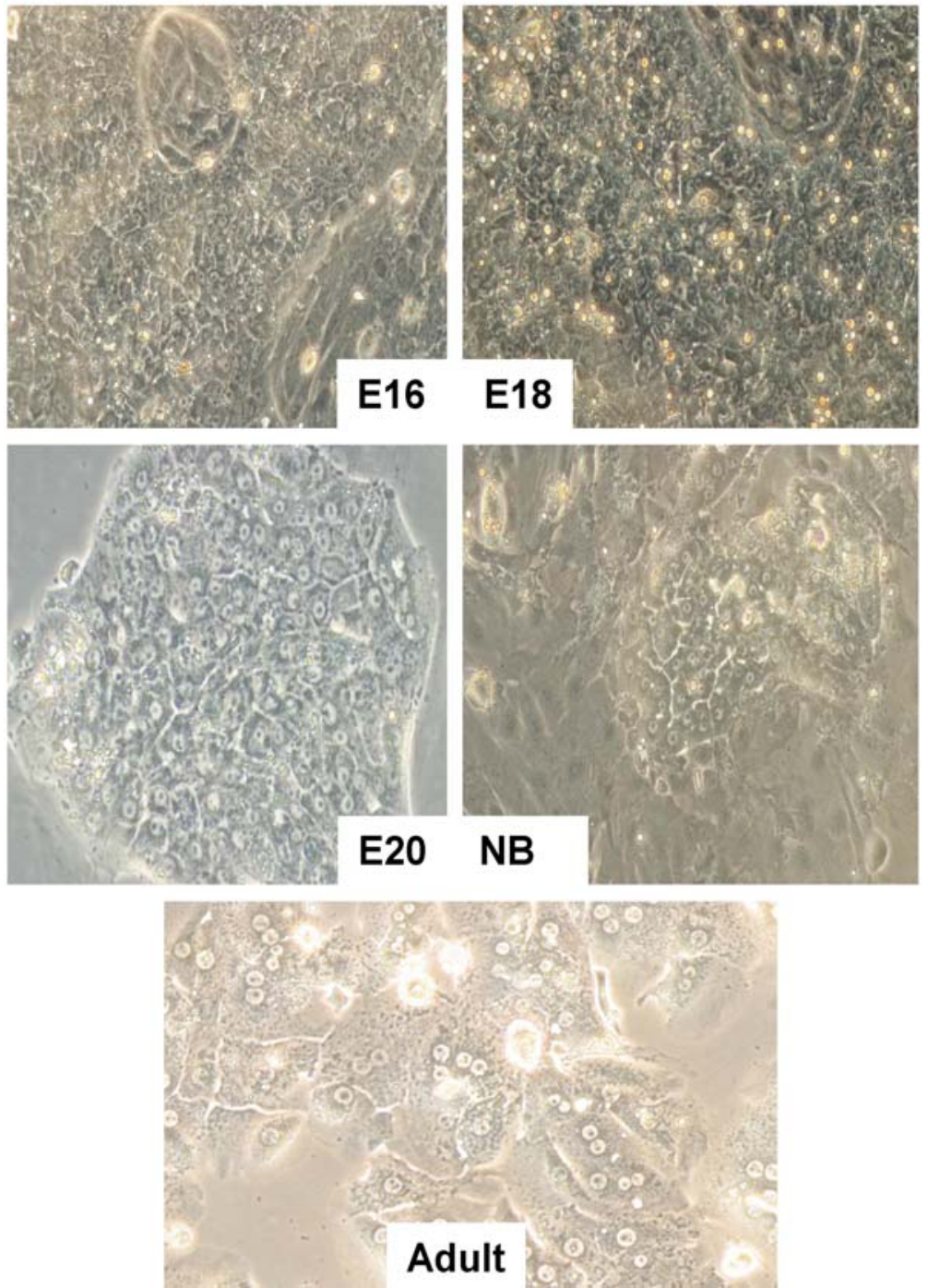

Figure 5: Phase-contrast microscopic pictures of primary rat hepatoblasts isolated at developmental stages E12, E14, E16, E18, E20 and primary hepatocytes derived from new born (NB) and adult liver. Original magnification $x 200$. 


\subsection{Albumin and AFP gene expression at the time of hepatic specification}

During rat embryogenesis the liver bud begins to develop at 10 days of gestation (E10). This is the time when different signals like bone morphogenetic proteins (BMPs), fibroblast growth factors (FGFs) and transcription factors such GATA-4 and Foxa induce the differentiation of endodermal cells into embryonic hepatic cells (Serls et al., 2005; Deutsch et al., 2001; Zaret, 1996; Zhao et al., 2005). The first molecular evidence for liver development is the expression of albumin and AFP.

\subsubsection{Identification of albumin and AFP mRNA expression in ventral foregut}

At 10 days of gestation, the time of initiation of hepatic specification, AFP was strongly expressed in the ventral foregut region (Fig. 6A). A single cell suspension of ventral foregut endoderm was performed and the cells were cultured for two days. In vitro in situ hybridization revealed that the ventral foregut region contains albumin and AFP mRNA expressing cells (Fig. 6B), which are surrounded by cells which shown no albumin- and AFPmRNA-expression. Albumin mRNA was expressed in cultured Hepatocyte, however, no reaction with the sense albumin probe was observed (Fig. $6 C)$. Albumin and AFP mRNA expression in the ventral foregut region was confirmed with real time PCR experiment. As shown in figure $8(A, B)$, the cells derived from ventral foregut region could express albumin and AFP mRNA. 

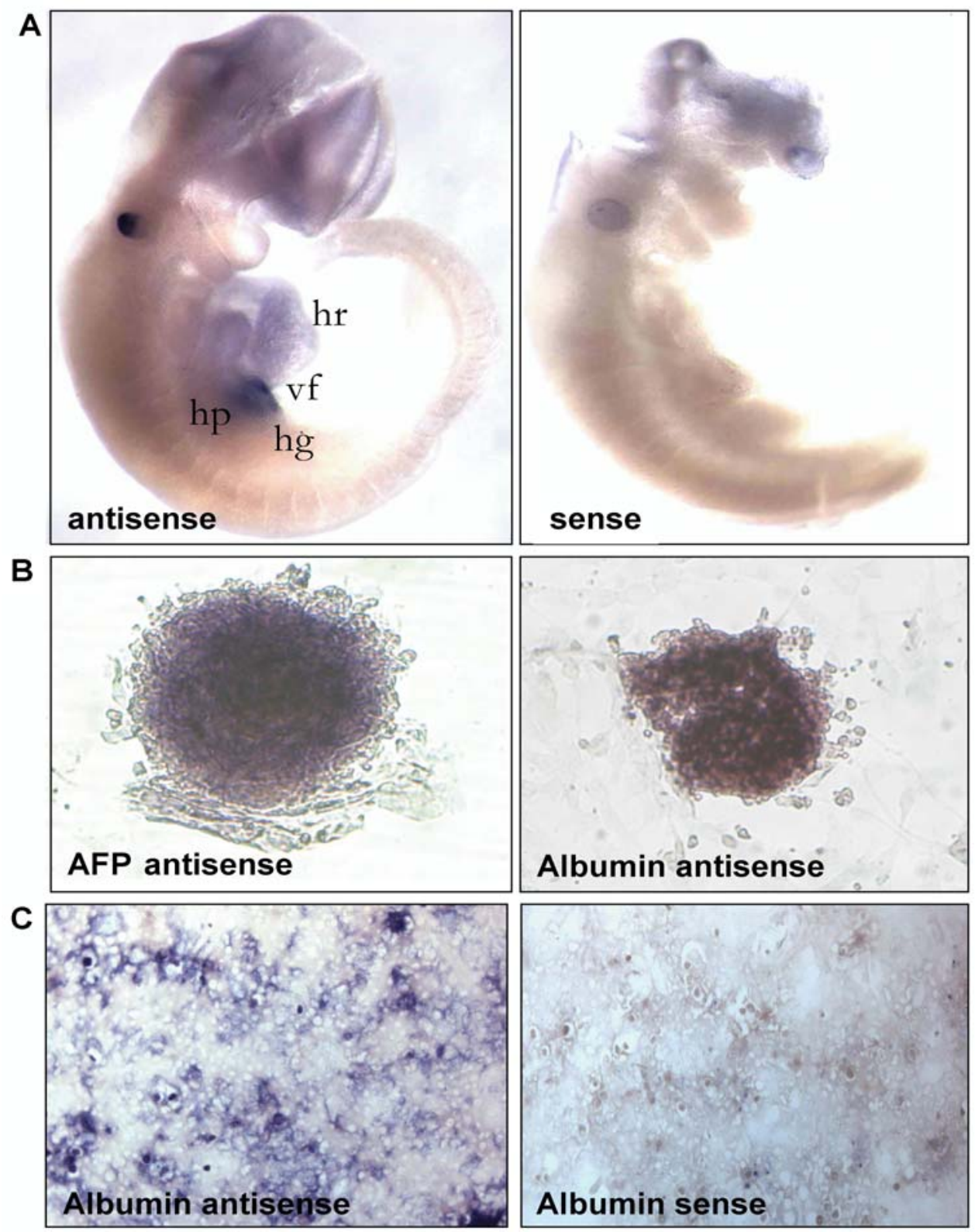

Figure 6: (A) AFP mRNA-Expression in ventral foregut region assessed by whole mount in situ hybridization. Albumin and AFP mRNA-expression in explanted endodermal cells (B) detected by in vitro in situ hybridization. Rat embryos at 10 days of gestation and cultured endodermal cells derived from ventral foregut were incubated with DIG-labelled sense and antisense probes of albumin and AFP as described in materials and methods. After incubation with alkaline phosphatase (AP)-conjugated anti DIG antibody, signals were visualised using BCIP/NBT. As positive control, the hepatocytes (C) were incubated with albumin sense and antisense probes. hr: heart, vf: ventrale foregut, $\mathrm{Hg}$ : hindgut, hp: hepatic primordium. 


\subsubsection{Synthesis and secretion of albumin and AFP by endodermal cells derived from ventral foregut.}

So far, immunological techniques failed to detect albumin and AFP expression on protein level in viable endodermal cells generated from ventral foregut. Hence, a sensitive method, radioactive biosynthetic labelling, was used to assess synthesis and secretion of albumin and AFP proteins. Endodermal cells derived from ventral foregut were kept in culture for two days and then overnight radioactively labelled with ${ }^{35} \mathrm{~S}$ methionine contained in the culture medium. Albumin and AFP were immunoprecipitated from $500 \mu \mathrm{l}$ supernatants (extracellular) and celllysates (intracellular) of the cell culture using anti-albumin or anti-AFP polyclonal antibodies. As positive control we used $50 \mu \mathrm{l}$ supernatants and cell-lysates from labelled hepatocyte and hepatoblats at 12 days of gestation. Cultured endodermal cells were found to synthesise and secrete biosynthetically labelled albumin and AFP (Fig. 7(A; B)). Interestingly, in $500 \mu \mathrm{l}$ of supernatants and cell-lysates of labelled endodermal cells, a low synthesis and secretion of albumin and AFP proteins were observed. In contrast, in $50 \mu \mathrm{l}$ of supernatants and celllysates of labelled hepatocyte and hepatoblats a high synthesis and secretion were observed. Endodermal cells derived from venral foregut could synthesise and secrete more AFP than albumin. This finding was confirmed at the RNA-level by real time PCR analysis (Fig. 8 (A; B)) 


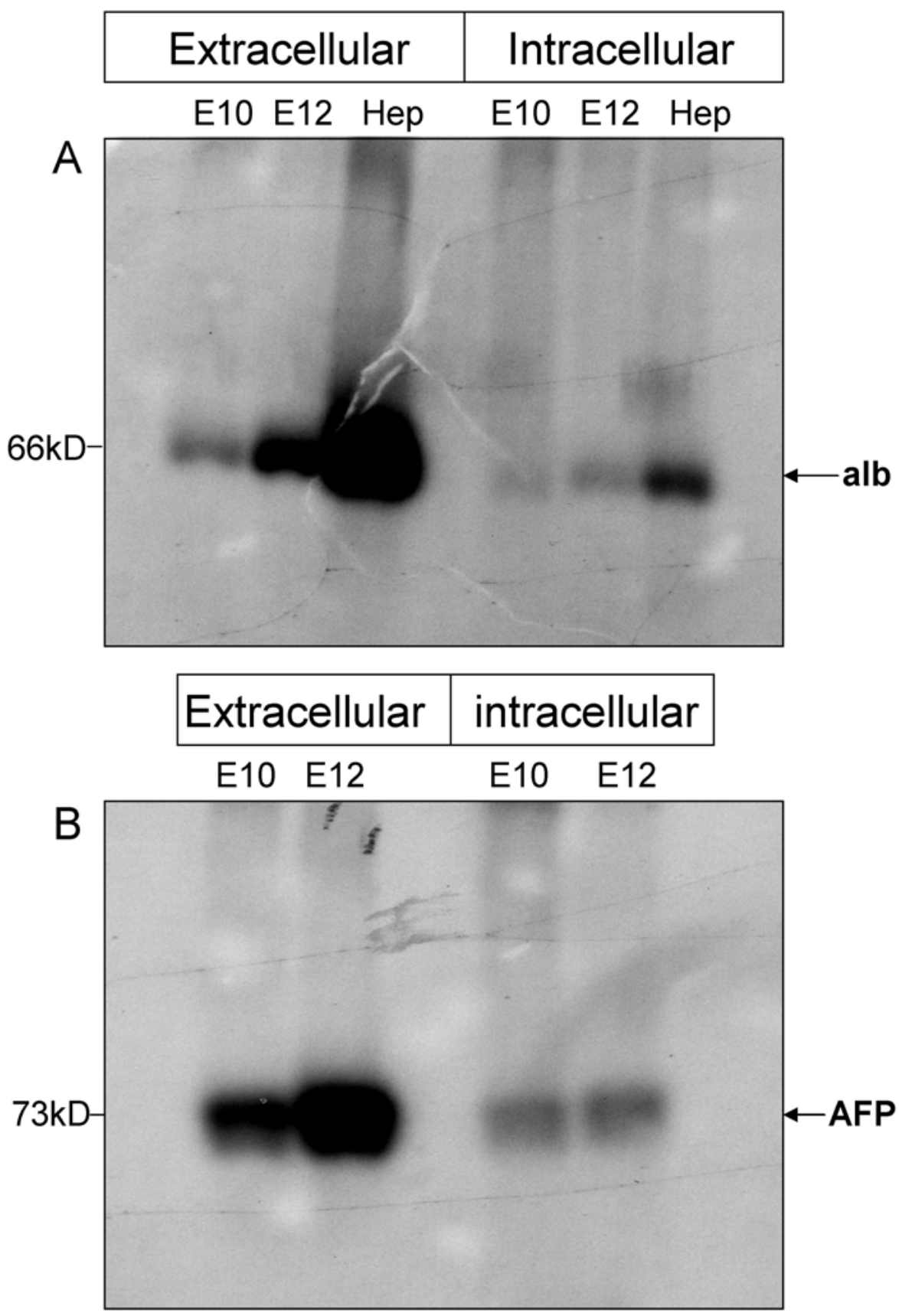

Figure 7: Synthesis and secretion of Albumin (A) and alpha-fetoprotein (B) by endodermal cells derived from ventral foregut region. Endodermal cells, hepatoblasts and hepatocytes were radioactive labelled with ${ }^{35} \mathrm{~S}$ methionine. $500 \mu \mathrm{l}$ of cell-lysates (intracellular) and supernatants (extracellular) were used for Immunoprecipitation of albumin and AFP from labelled endodermal cells. As positive control, we used $50 \mu \mathrm{l}$ of cell-lysates and supernatants from labelled hepatoblasts and hepatocytes for immunoprecipitation of albumin and AFP. The immuncomplexes were analysed by SDS-PAGE. 
A

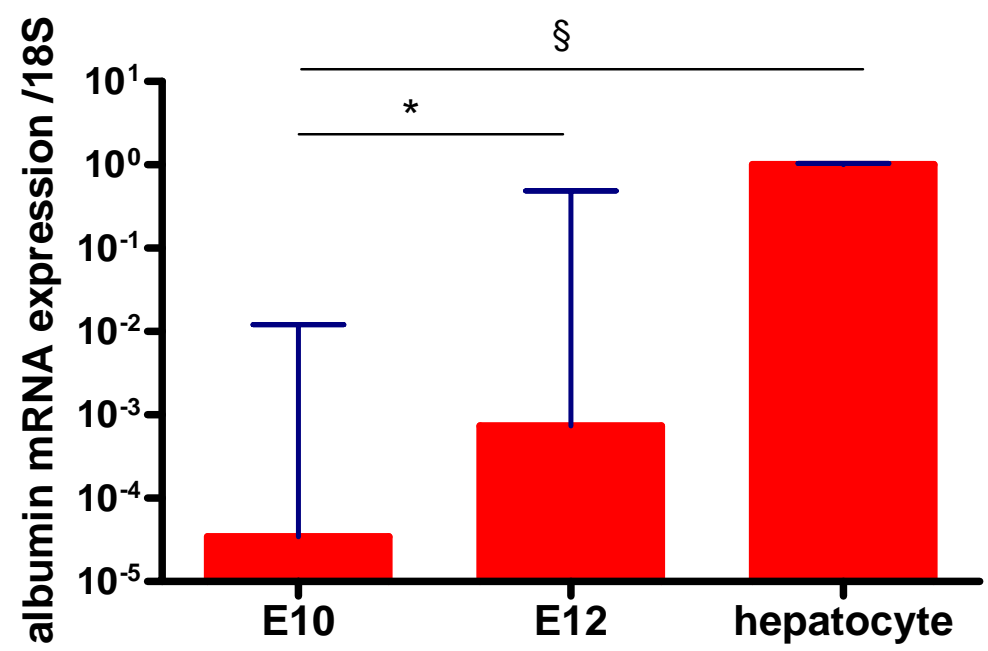

B

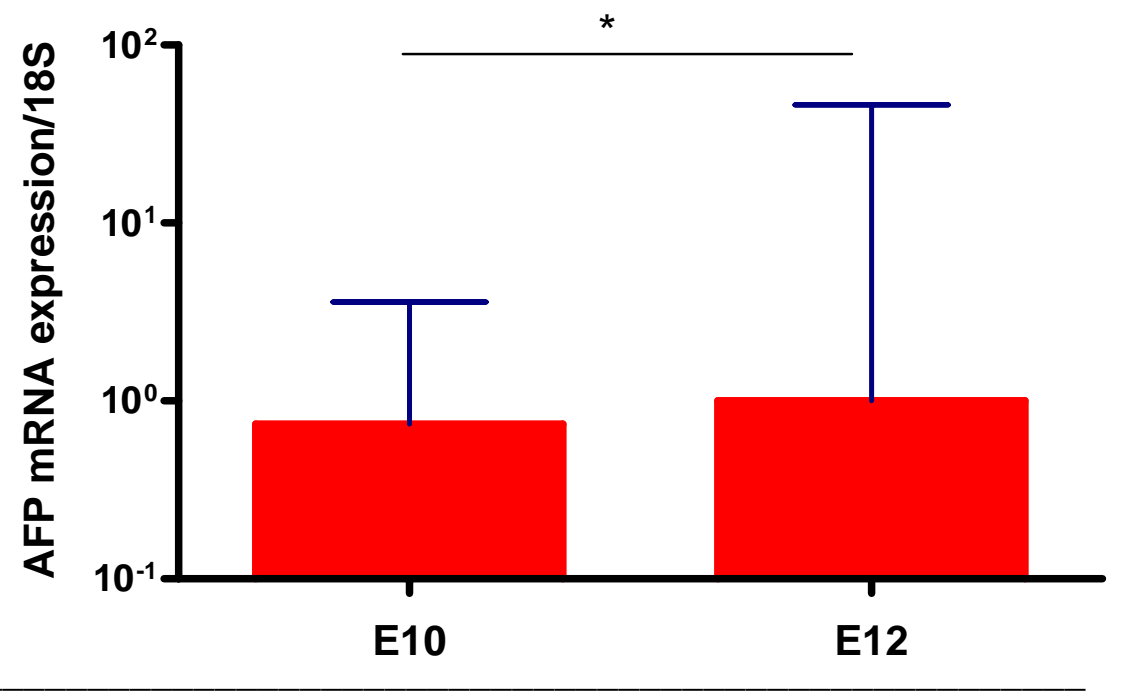

Figure 8: Real-time-PCR analysis of mRNA extracted from cultured endodermal cells, hepatoblasts and hepatocyte. Albumin (A) and AFP (B) mRNA were expressed in cultured endodermal cells derived from ventral foregut. As positive control, mRNA from hepatoblasts at 12 days of gestation and hepatocytes were used. cDNA from endodermal cells and hepatoblasts were prepared using Fastlane Cell cDNA kit (Qiagen). The expression was normalized with the endogenous control of ribosomal RNA 18S. Error bars represent S.E.M, $n=3$. Statistically significant difference ( ${ }^{\star} \mathrm{P}<0.05 ; \S<0.05$ student's $t$-test) compared to positive control (hepatoblasts and hepatocytes). 


\subsection{Characterization of endodermal cells generated from ventral foregut}

The endodermal cells derived from ventral foregut were characterized by assessing the expression of endodermal and hepatic markers as well as transcription factors involved in liver development. Positive cells for HNF4alpha were detected in cultured clustered endodermal cells by immunofluorescence experiment (Fig. 9). In addition real-time-PCR analysis revealed that explanted endodermal cells could express HNF4alpha, Prox1, beta-catenin, BMP-4, Foxa-2 and GATA-4 (Fig. 10).
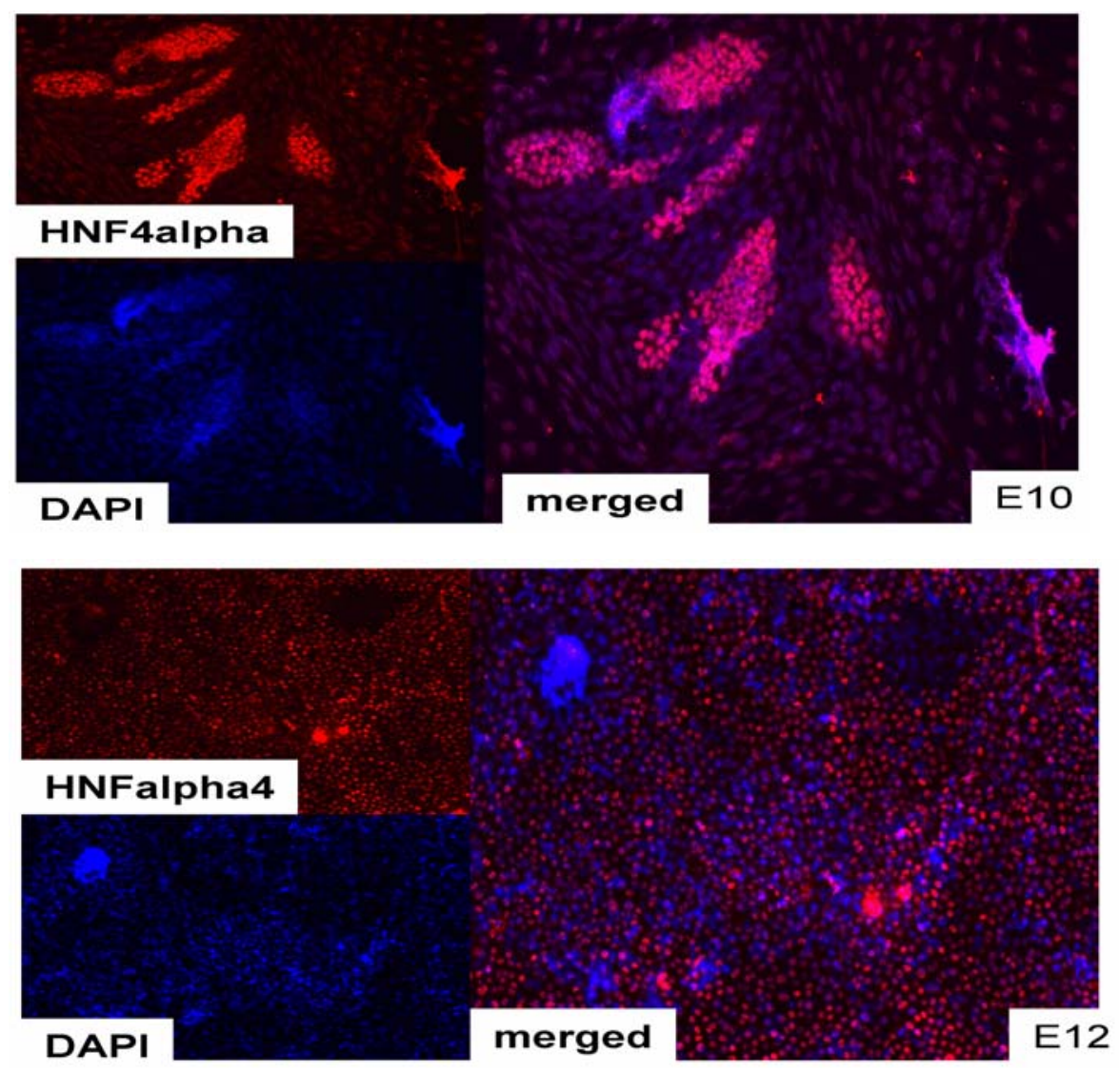

Figure 9: Immunofluorescence staining display HNF4-aplha-expression in cultured clustered endodermal cells and in hepatoblasts derived from 12 days of gestation. Counterstaining of nuclei with DAPI (blue). Original magnification $\times 100$ 

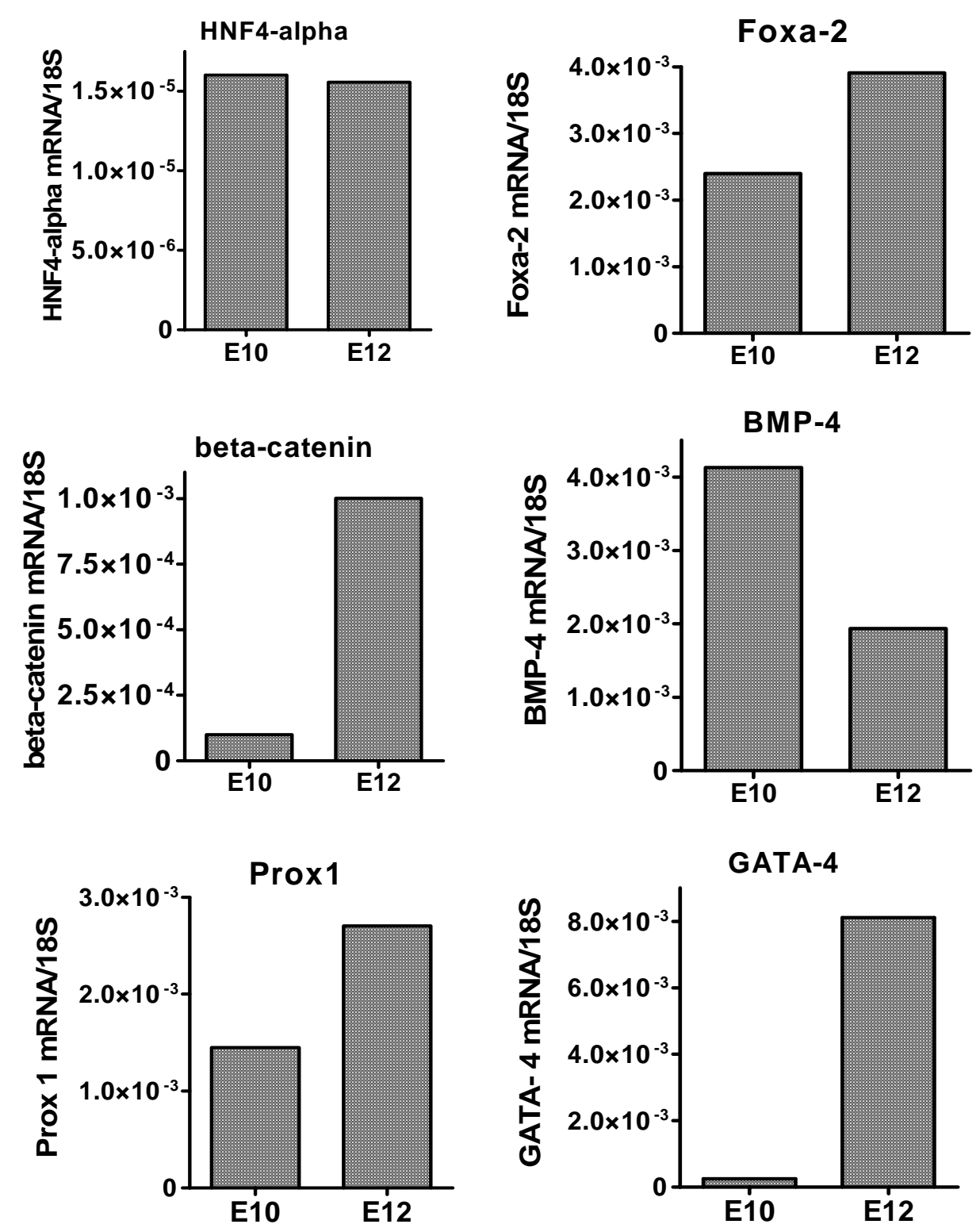

Figure 10: mRNA-expression levels of HNF4-alpha, beta-catenin, Prox1, Foxa-2, BMP-4 and GATA-4 in cultured endodermal cells and hepatoblasts from 12 days of gestation measured by real-time-PCR experiment. cDNA from cultured endodermal cells and hepatoblasts from 12 days of gestation were prepared using Fastlane Cell cDNA kit. The expression was normalized with the endogenous control of ribosomal RNA 18S. 


\subsection{Cellular analysis of albumin and AFP expressing cells during liver development}

The embryonic liver appears at 12 days of gestation, when albumin and AFP mRNA as well as theirs coding proteins are expressed. The ratio of albumin and AFP producing cells either to total liver cells or to proliferating cells was analysed during liver development. For this purpose, at each developmental stage in situ hybridization was performed using albumin and AFP probes combined with proliferating cell nuclear antigen (PCNA) peroxidase immunostaining.

Albumin and AFP positive cells were distributed homogeneously during liver development (Fig. 11 and Fig. 12). A few negative cells which represents hematopoitic or endothelial cells were observed. Adult liver could not express AFP but albumin is high expressed. The same expression pattern has been previously observed in rat liver during prenatal (17 to 21 days of gestation) and postnatal life ( 1 to 5 weeks old neonates) (Poliard et al., 1986).

During embryonic and foetal stage (from E12 up to E18), about 50\% of liver cells expressed albumin and AFP. From E18 up to birth, albumin and AFP positive cells developed inversely, we observed an increase of albumin positive cells and a decrease of AFP positive cells, which disappears in adult liver (Fig. 13). 

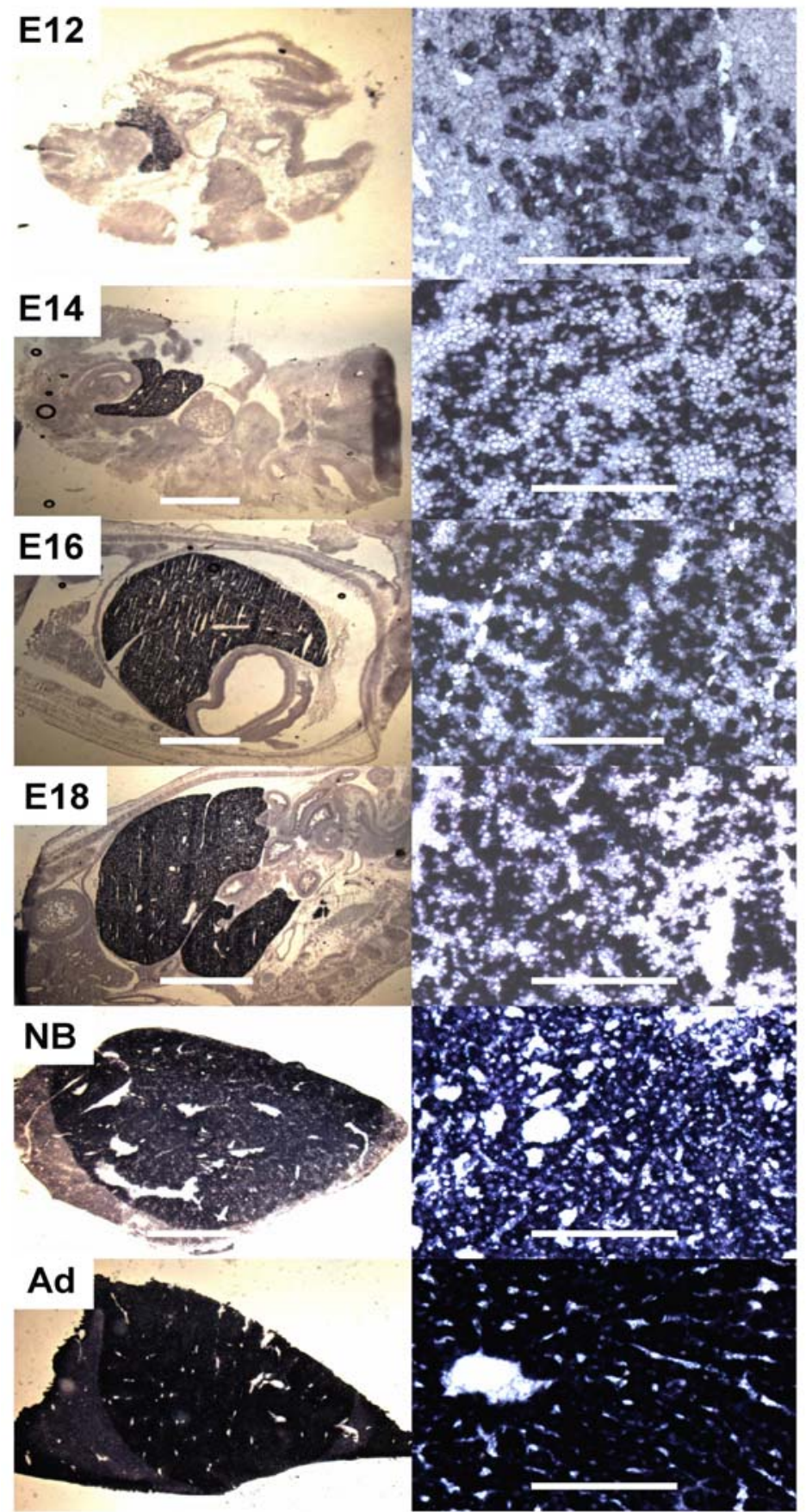

Figure 11: Albumin mRNA-expression during liver development assessed by in situ hybridization. Cryo-sections $(10 \mu \mathrm{m})$ of rat embryos prepared at developmental stages E12, E14, E16, E18, new born and adult liver were incubated with DIG-labelled antisense riboprobe of albumin. After incubation with alkaline phosphatase (AP)-conjugated anti DIG antibody, the signals were visualised using BCIP/NBT.

(Bar = Magnification x 50; x 200). 


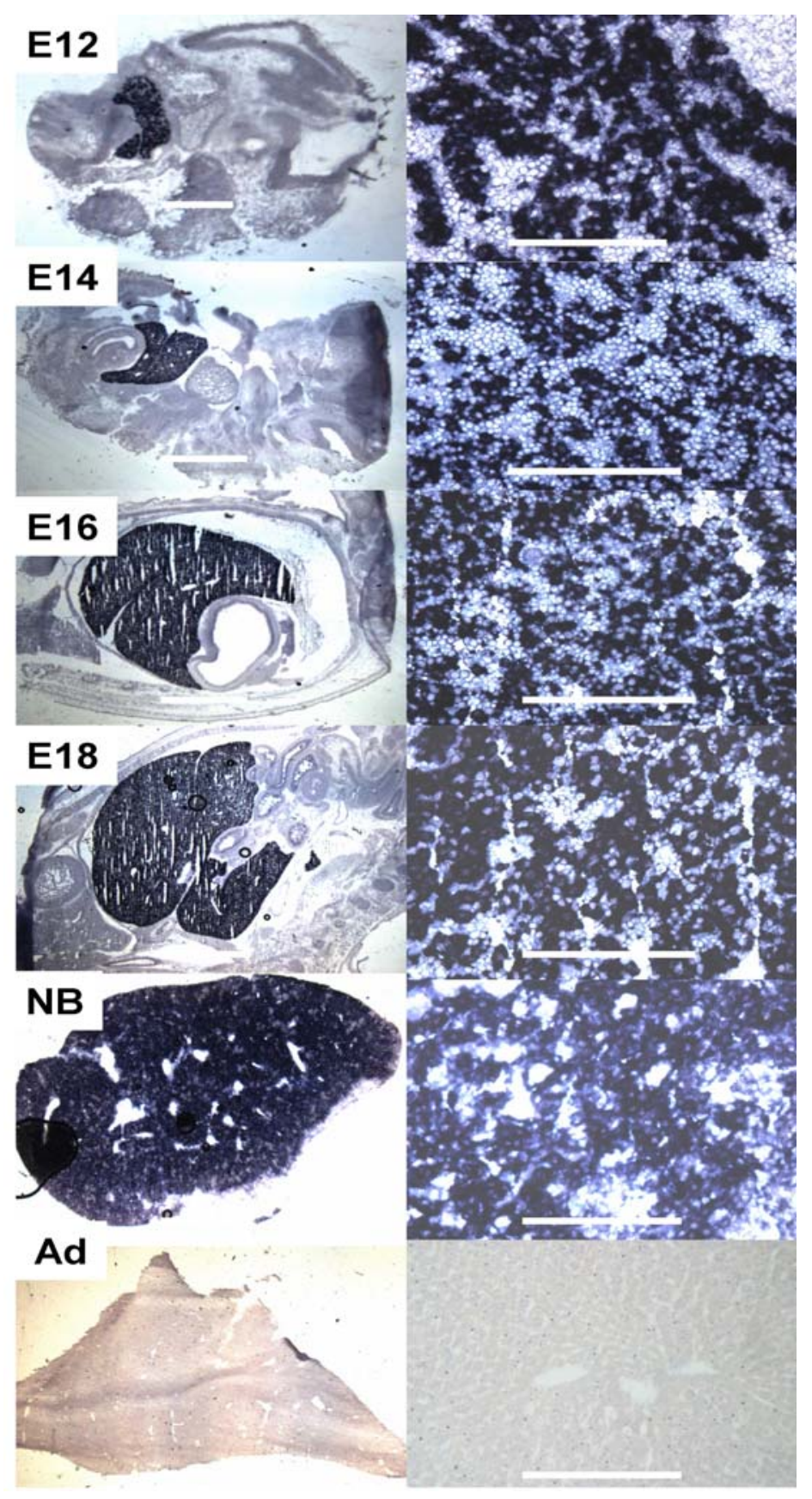

Figure 12: AFP mRNA-expression during liver development assessed by in situ hybridization. Cryo-sections $(10 \mu \mathrm{m})$ of rat embryos prepared at developmental stages E12, E14, E16, E18, new born and adult liver were incubated with DIG-labelled antisense riboprobe of AFP. After incubation with alkaline phosphatase (AP)-conjugated anti DIG antibody, the signals were visualised using BCIP/NBT. (Bar = Magnification x 50; x 200). 


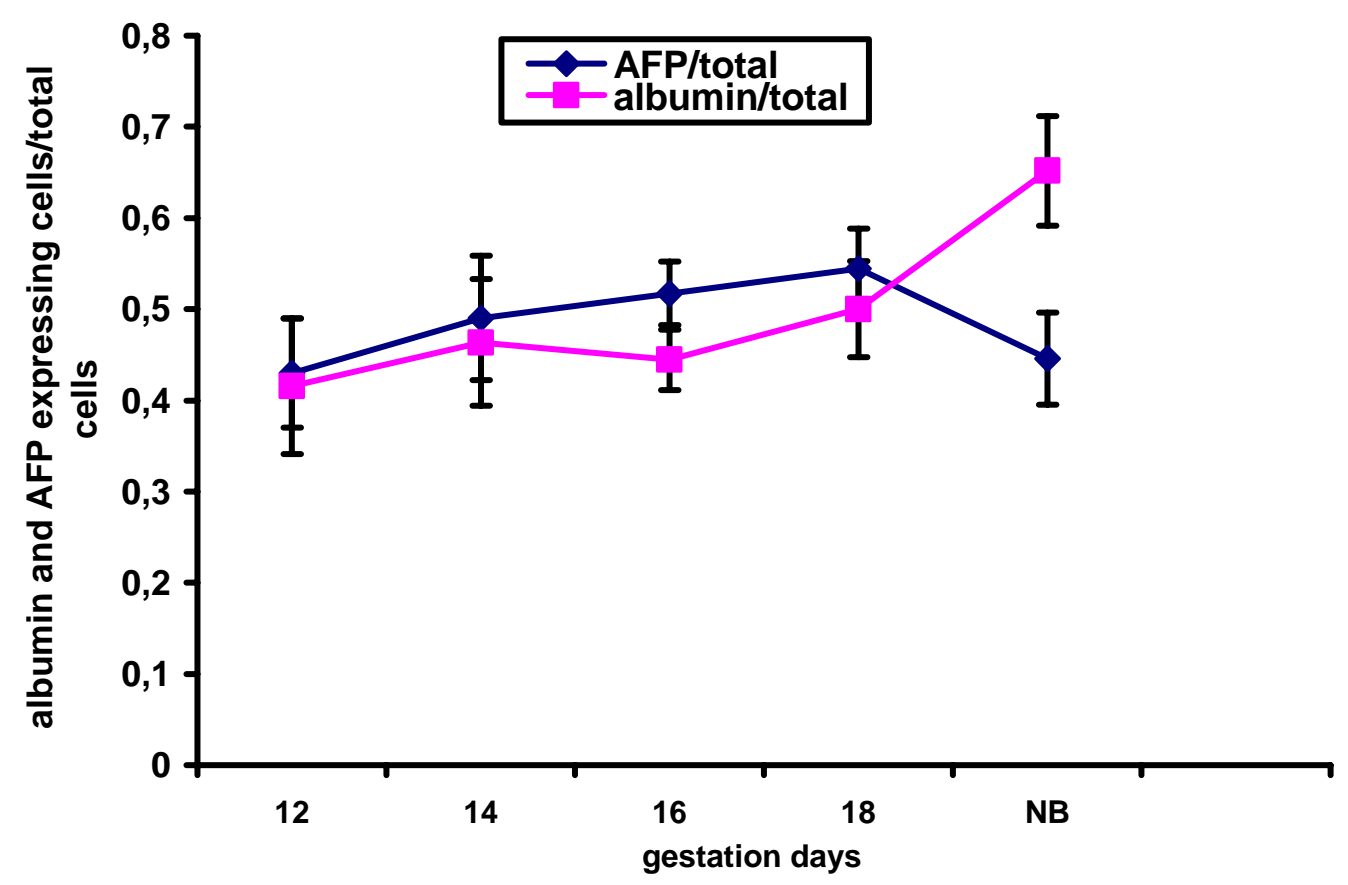

Figure 13: The ratio of albumin and AFP producing cells to total cells during liver development. Albumin and AFP positive cells were identified by in situ hybridization (as shown in figure 11 and 12) and counted under microscope using a shaded ocular, or by application of Image $\mathrm{J}$ software (Wayne Rasband, $\mathrm{NIH}$, USA). Error bars represent S.E.M., $\mathrm{n}=3$. The significance $(P<0.05)$ was analysed by ANOVA

Cell proliferation in developing liver was measured by immunoreactions with proliferating cell nuclear antigen (PCNA) in embryonic and foetal livers at E12, E14, E16, E18 and in livers from new born and adult state. A high number of PCNA-positive cells were observed at E12, E14 and E16. Fewer PCNA-positive cells were observed at E18 and new born rats. Far fewer PCNA-positive cells were observed in adult liver (Fig. 14). In developing liver, the ratio of albumin and AFP producing cells to proliferating cells shows an increase during embryonic and foetal stages. At E18 the ratio of AFP producing cells to proliferating cells reaches the 
maximum, followed by a decrease at birth. In contrast the ratio of albumin producing cells to proliferating cells increase continuously (Fig. 15)
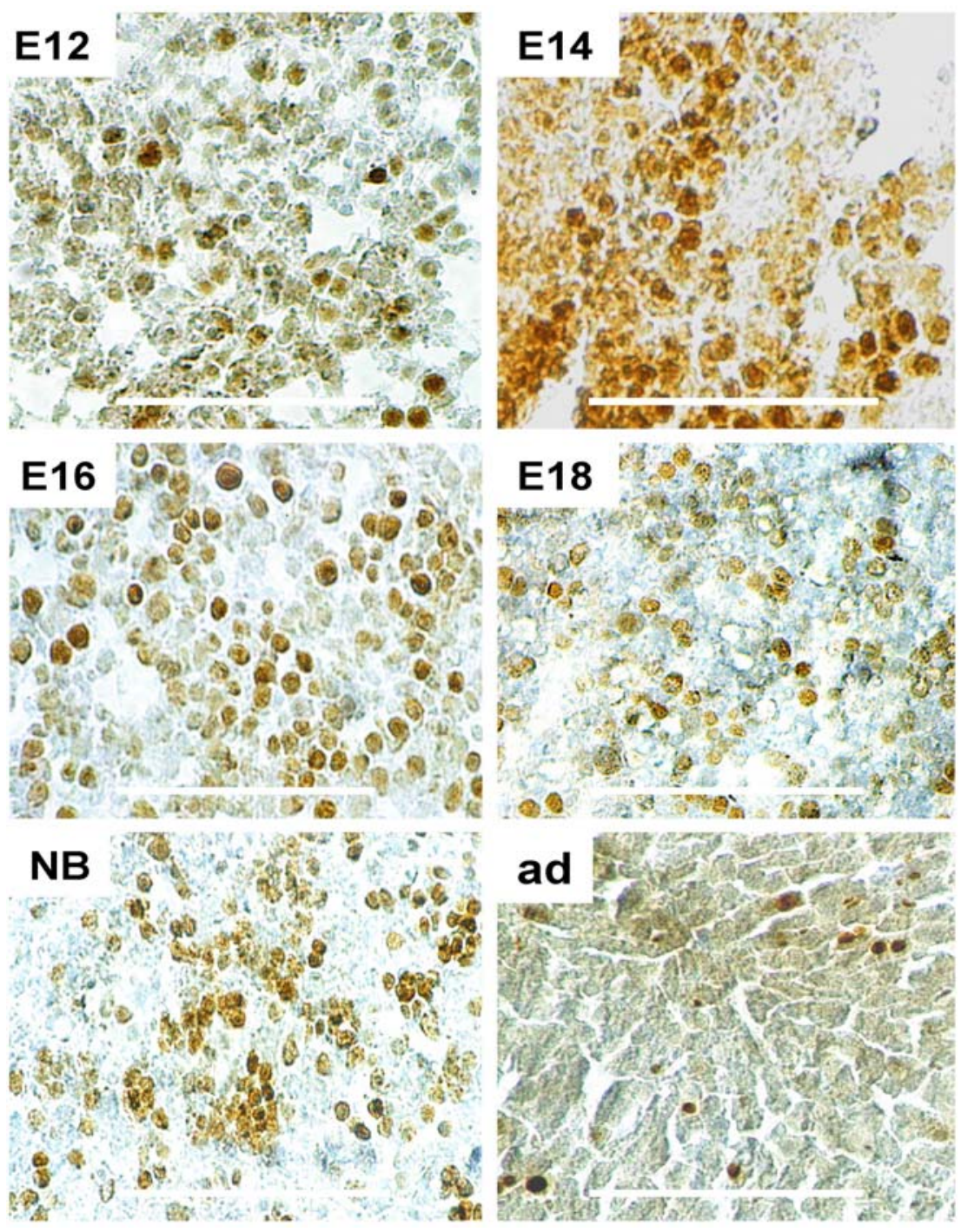

Figure 14: Proliferation estimated by the number of positive cells expressing proliferating cell nuclear antigen (PCNA) during liver development. Cryo-sections $(5 \mu \mathrm{m})$ of rat embryos at developmental stages E12, E14, E16, E18, new born and adult liver were used. Immunreaction was detected by peroxidase-labelled second antibody. (Bar = Magnification $\times 200$ ). 


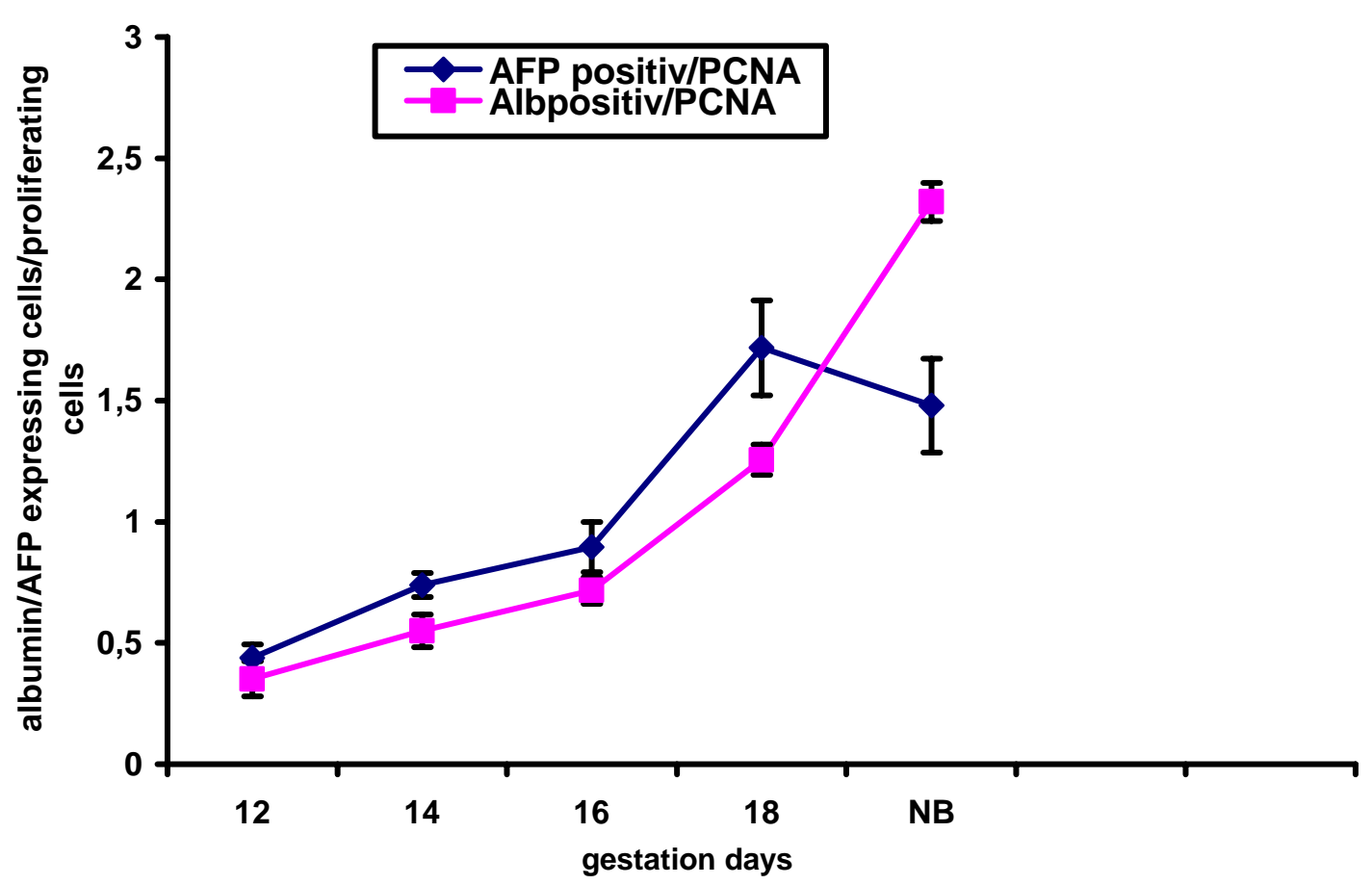

Figure 15: The ratio of albumin and AFP expressing cells to proliferating cells during liver development. Albumin/AFP and PCNA positive cells were identified by in situ hybridization (Fig. 11 and 12) and PCNA staining (Fig. 14) respectively. The positive cells were counted under microscope using a shaded ocular, or by application of Image J software (Wayne Rasband, NIH, USA). Error bars represent S.E.M., $n=3$. The significance $(P<0.05)$ was analysed by ANOVA

The co-localisation (co-expression) of albumin and AFP was observed at all developmental stages (from E12 up to birth). At birth, albumin could be produced by hepatocytes which express AFP anymore (Fig. 16). The coexpression of albumin and AFP genes in hepatoblasts was confirmed by in vitro in situ hybridization, performed in cultured hepatoblasts at 12, 14 and 18 days of gestation. As shown in figure 17, the hepatoblasts could express albumin and AFP mRNA. 


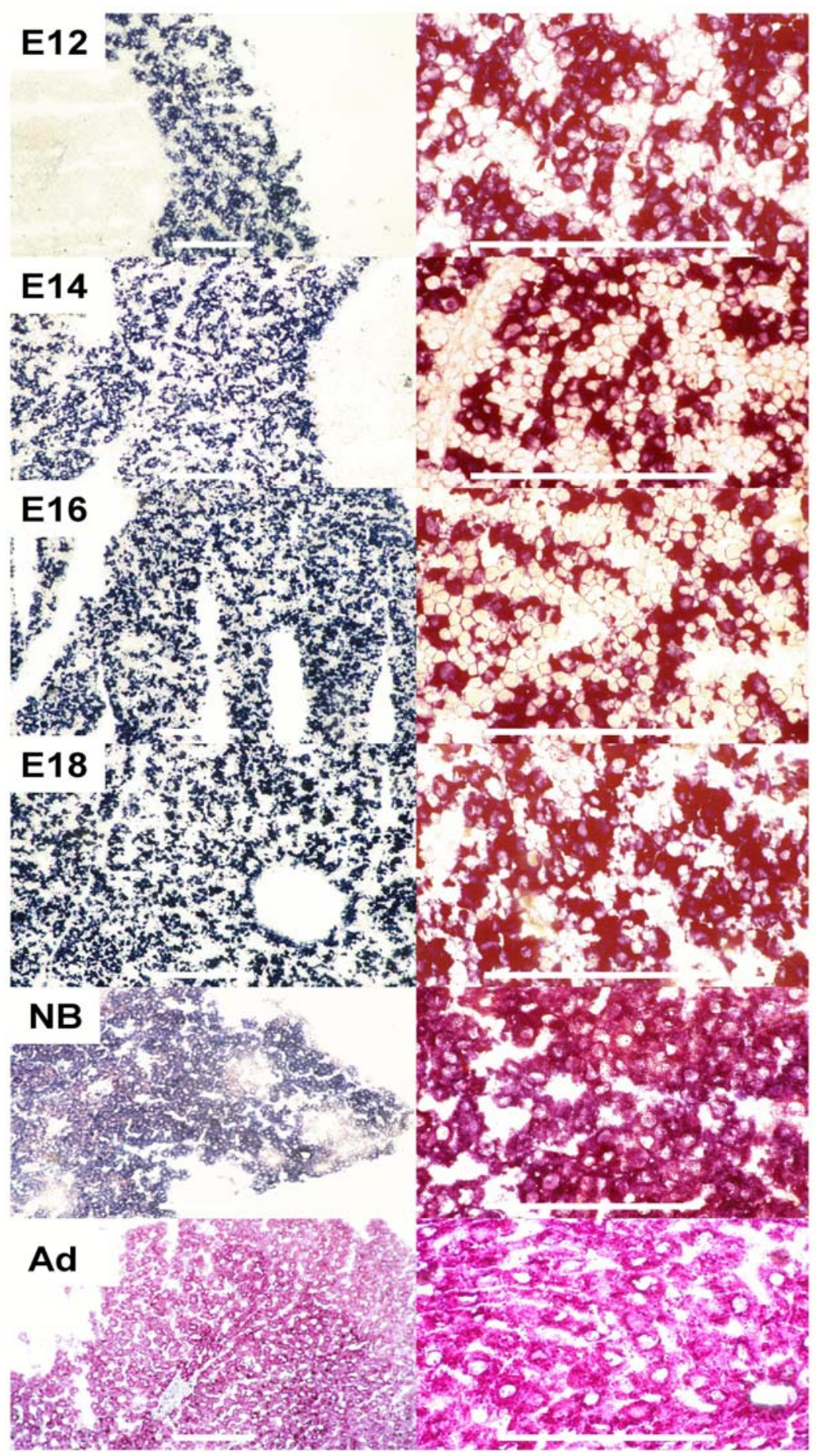

Figure 16: Co-localisation of albumin and AFP mRNA during liver development. Cryo-sections $(10 \mu \mathrm{m})$ of rat embryos at developmental stages E12, E14, E16, E18 as well as new born and adult livers were incubated with DIG-labelled antisense riboprobe of AFP (blue) and Fluorescein-labelled antisense riboprobe of albumin (red).

(Bar = Magnification x 100; x 200). 


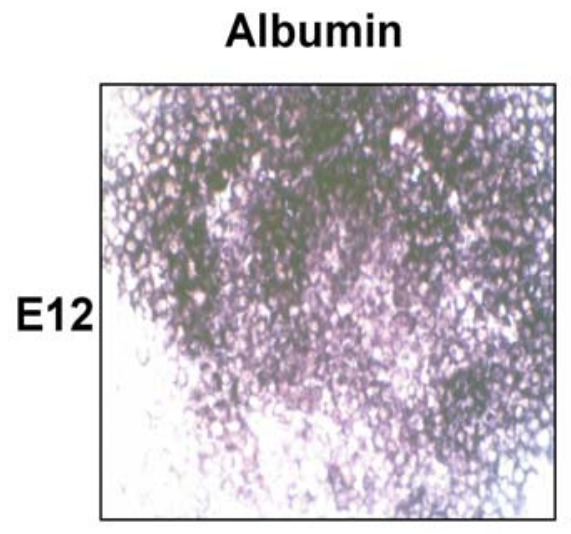

Alpha-fetoprotein
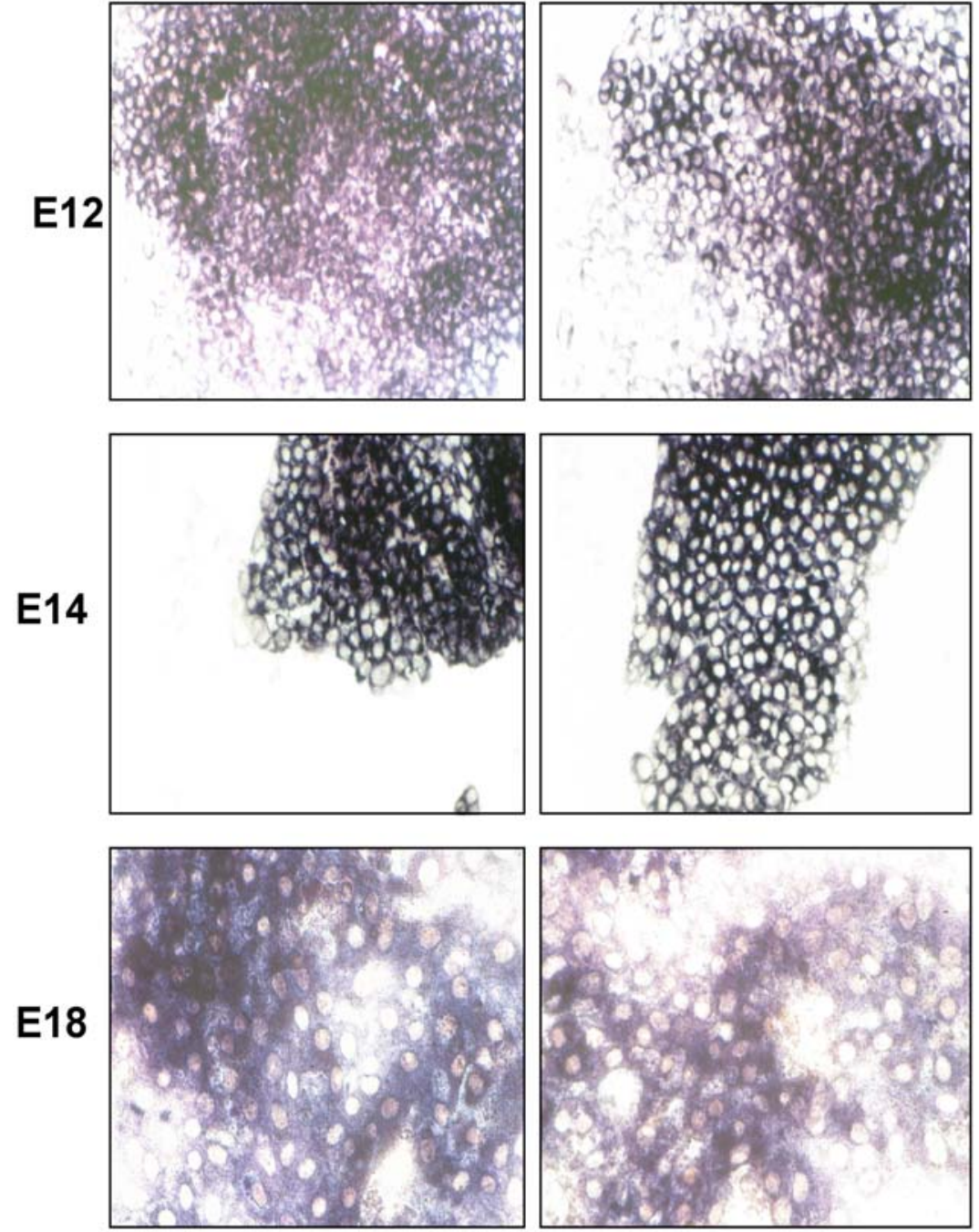

Figure 17: Albumin and AFP mRNA expression in cultured hepatoblasts during liver development. Hepatoblasts derived from developmental stages E12, E14 and E18 were cultured on labTecks for two days and incubated with DIG-labelled antisense riboprobe of albumin and AFP. (Magnification x 200). 


\subsection{Quantitative analysis of albumin and AFP mRNA expression during liver development}

In situ hybridization experiments have provided more insight about cellular distribution of albumin and AFP expressing cells during liver development. Quantitation of albumin and AFP mRNA was obtained by real-time PCR experiment using a reverse transcripted RNA extract from whole liver (in vivo) and from hepatoblasts (in vitro) derived from developmental stages E12, E14, E16, E18, E20, new born and adult.

In whole liver (in vivo), albumin mRNA was maintained from 12 up to 16 days of gestation. However, AFP mRNA displayed a high expression at E12 followed by a decrease at E14 and remained unchanged until 16 days of gestation. Both genes reach a peak at 18 days of gestation. A slight decrease at E20 followed by an increase of albumin mRNA at birth and during postnatal stage. AFP mRNA decrease continuously during fetal stage, at birth and during postnatal life (Fig. 18).

In hepatoblasts (in vitro), it was observed an increase of albumin and AFP mRNA during embryonic stage. AFP mRNA reached a peak at 16 days of gestation and then decreased, while albumin mRNA reached a peak at 18 days of gestation followed by a slight decrease at E20 and then increased at birth and during postnatal life (Fig. 19). 
A

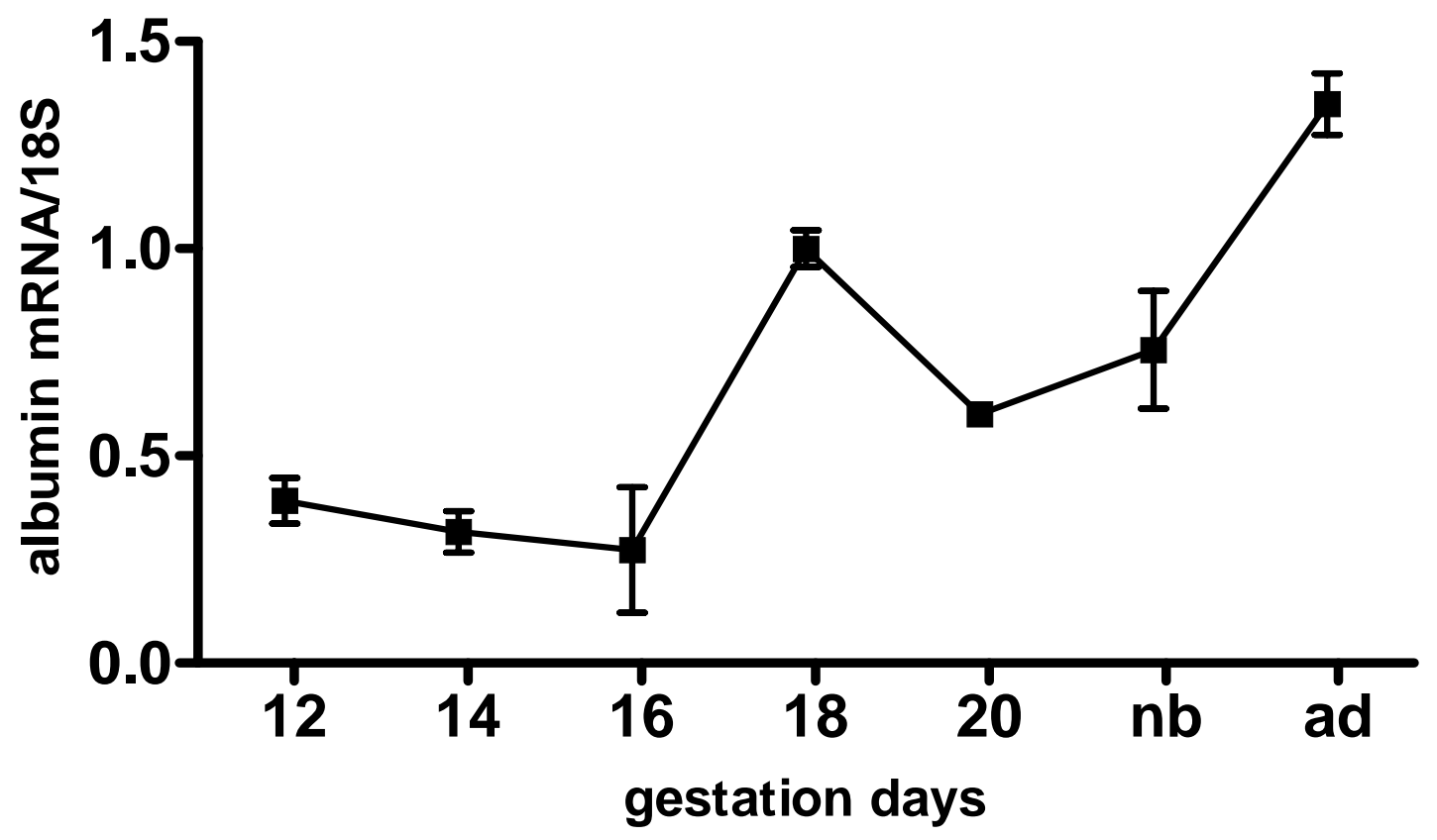

B

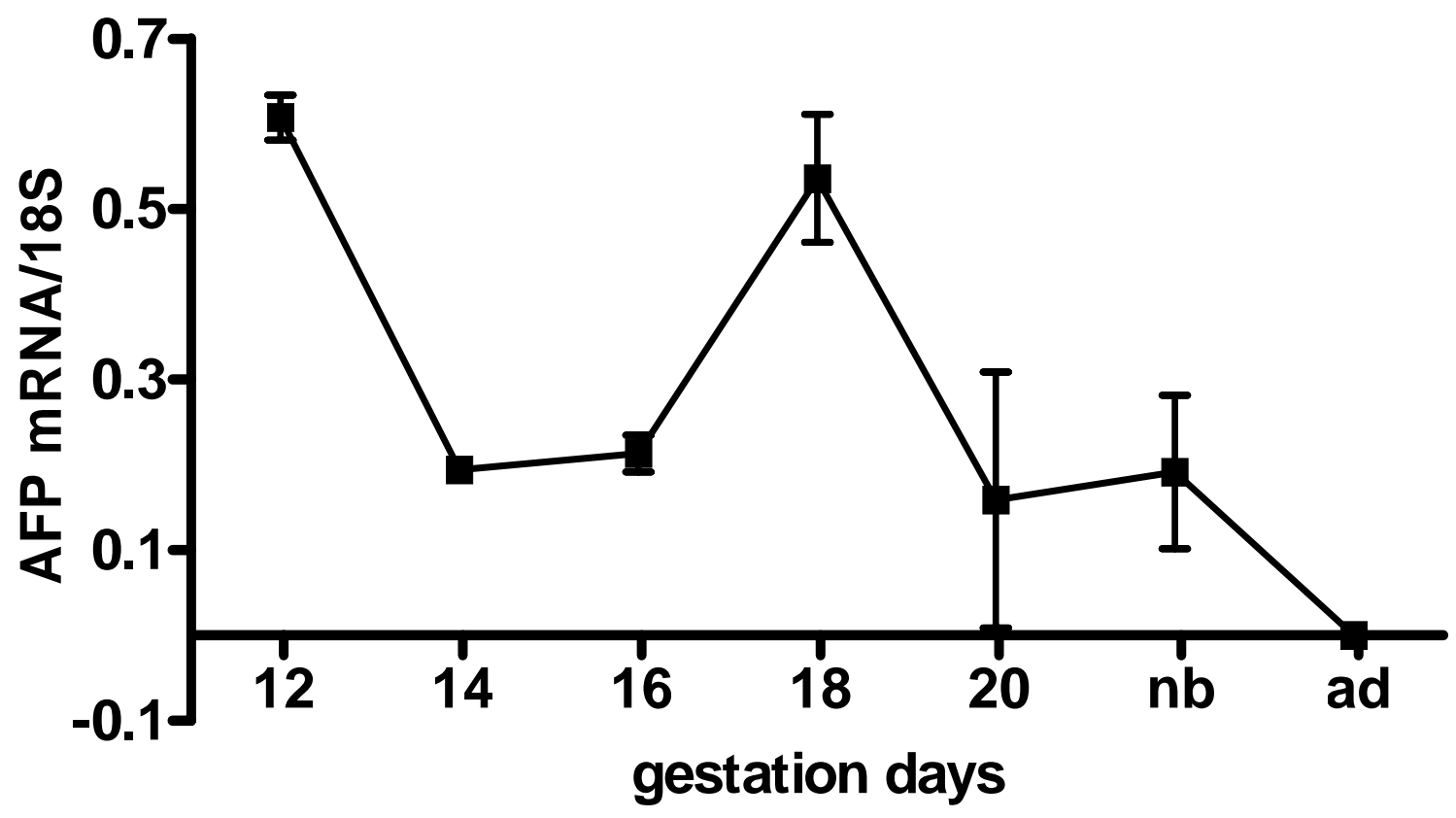

Figure 18 Quantitative analysis of albumin (A) and AFP (B) mRNA expression in whole liver (in vivo) during liver development using realtime-PCR experiment. The expression was normalized with the endogenous control of ribosomal RNA 18S. Error bars represent S.E.M., $n=3$. The significance $(P<0.05)$ was analysed by ANOVA. 
A
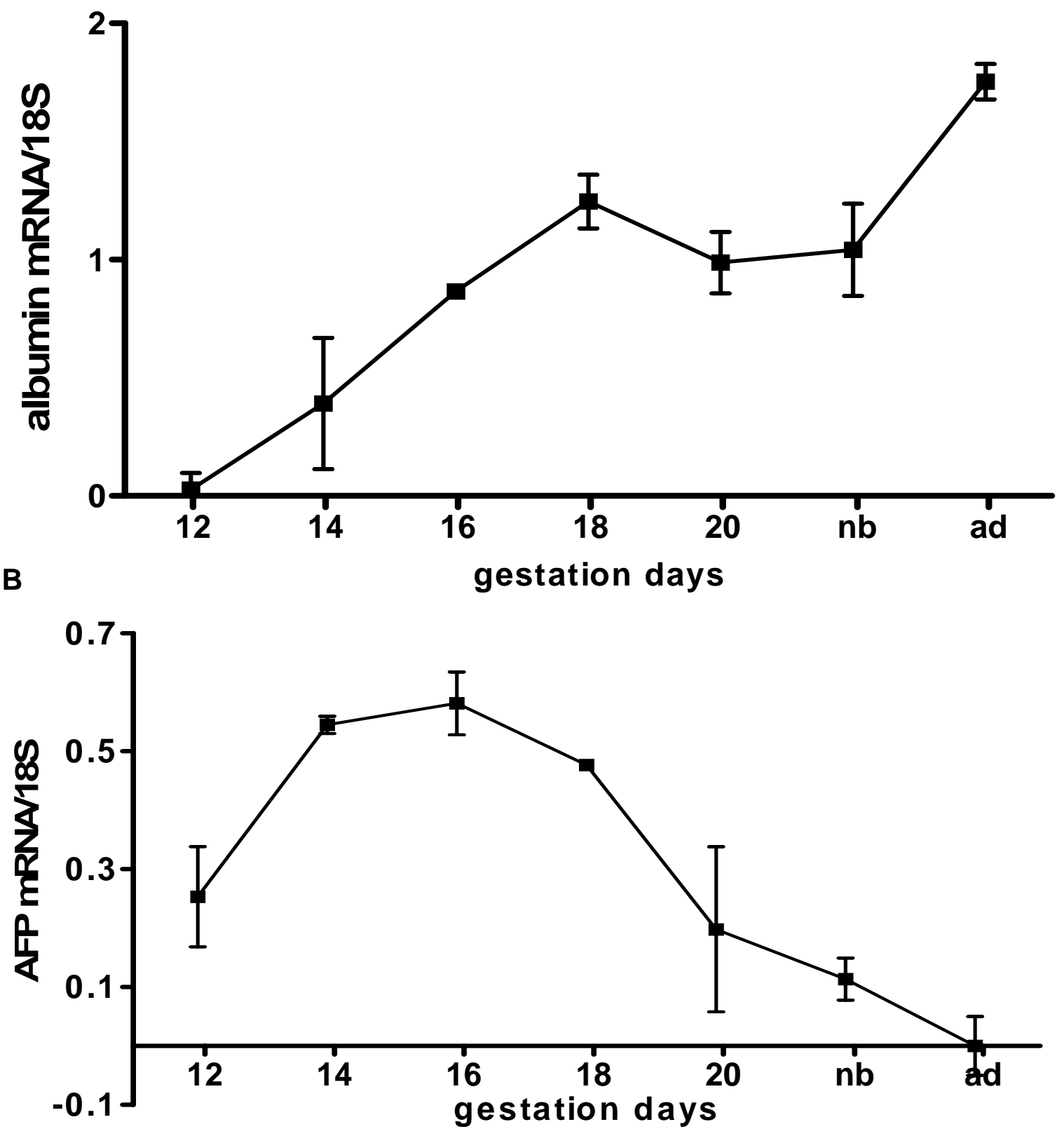

Figure 19: Quantitative analysis of albumin (A) and AFP (B) mRNA expression in cultured hepatoblasts (in vitro) during liver development using real-time-PCR experiment. The expression was normalized with the endogenous control of ribosomal RNA 18S. Error bars represent S.E.M., $n=3$. The significance was analysed by ANOVA $(P<0.05)$ 


\subsection{Kinetics for synthesis and secretion of albumin and AFP during liver development}

The kinetics for synthesis, as well as secretion of albumin and AFP was investigated by radioactive biosynthetic labelling method in viable hepatoblasts and hepatocytes isolated from livers at E12, E14, E16, E18, E20, new born and adult rats. Newly synthesized proteins were endogenously labelled for $2 \mathrm{~h}$ with ${ }^{35} \mathrm{~S}$ methionine. Albumin and AFP were immunoprecipitated from supernatants (extracellular) and cell-lysates (intracellular). As shown in figure 20, during embryonic stage (E12 up to E16) synthesis and secretion of albumin and AFP remained relatively unchanged, approximately the same amount of albumin and AFP was released. At day 18 of gestation, an increase of synthesis and secretion of albumin and AFP was observed, followed by a decrease during prenatal stage (E20). At birth low levels of AFP were secreted, in contrast, albumin was secreted continuously. Hepatocyte generated from adult liver displayed a high synthesis and secretion of albumin, and a low secretion of AFP. The two polypeptides $68 \mathrm{kD}$ and $70 \mathrm{kD}$, which correspond to 2.1 $\mathrm{kb}$ AFP mRNA, were synthesised and secreted by hepatoblasts at all developmental stage.

During liver development, the kinetics of secretion of albumin and AFP in hepatoblasts was measured using pulse chase experiment. Hepatoblasts were kept in culture for two days, pulsed for $1 \mathrm{~h}$ with $100 \mu \mathrm{Ci} /$ well ${ }^{35} \mathrm{~S}$ methionine and then chased for 15, 30, 45, 60, 90, 120, 240 min. Mature hepatocyte needed short time to synthesize and secrete albumin. However, hepatoblasts isolated at early development stage needed more time to synthesize and secrete albumin. At 14 and 16 days of gestation albumin needed $30 \mathrm{~min}$ to be released but hepatoblasts from 18 days of gestation are comparable to mature hepatocyte. They needed only $15 \mathrm{~min}$ to secrete new synthesized albumin (Fig. 21A). AFP was secreted in 
hepatoblasts derived from developmental stage E12 after chase period of 45 min. At developmental stages E14, E16 and E18, AFP needed only 15 min to be secreted (Fig. 21B).

\section{E12 E14 E16 E18 E20 NB Ad}

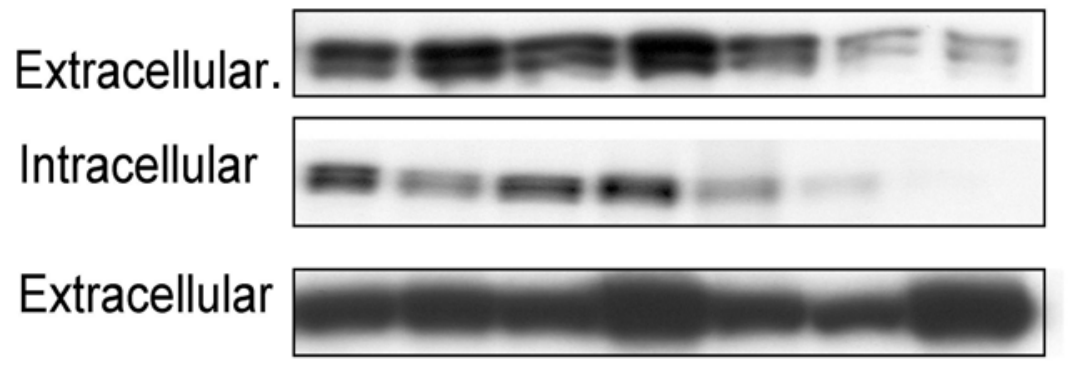

Intracellular

AFP

Albumin

Figure 20: Synthesis and secretion of albumin and alpha-fetoprotein (AFP) in viable hepatoblasts during liver development. Hepatoblasts and hepatocytes were cultured for two days and then radioactive labelled with ${ }^{35} \mathrm{~S}$ methionine. Albumin and AFP were immunopecipitated using polyclonal anti-albumin and anti-AFP antibodies. Immunocomplexes were analysed by SDS-PAGE. Celllysates (Intracellular) and supernatants (extracellular) were used for immunoprecipitaion by taking into consideration samples with similar count. 
A

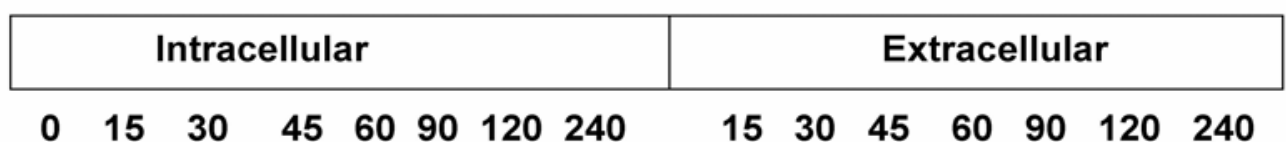

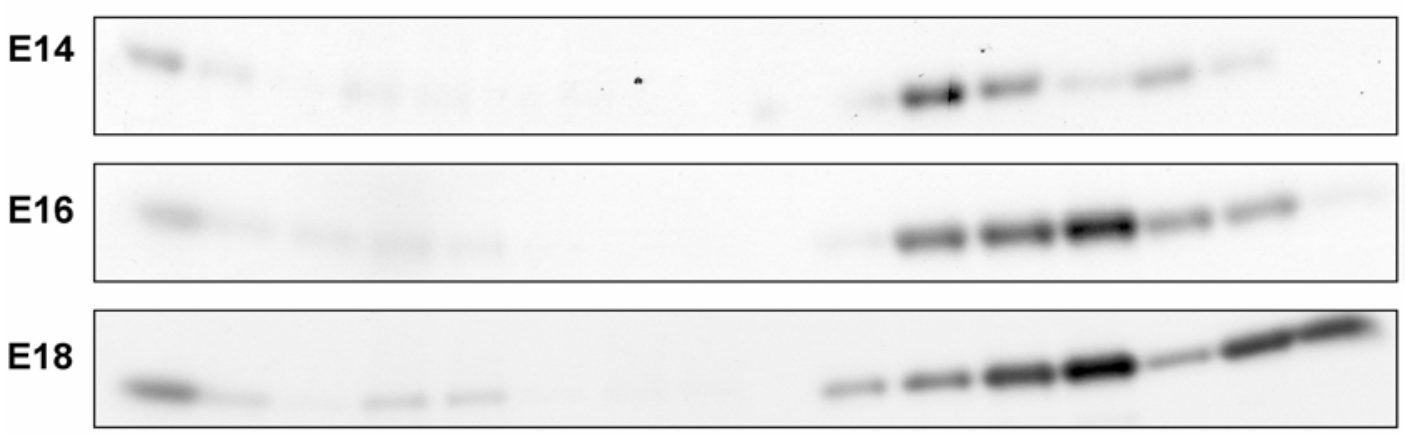

Ad

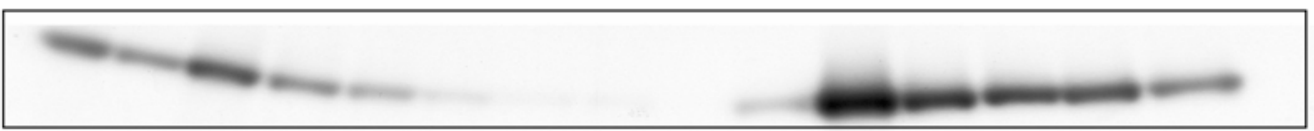

B

\begin{tabular}{|c|c|}
\hline Intracellular & Extracellular \\
\hline
\end{tabular}
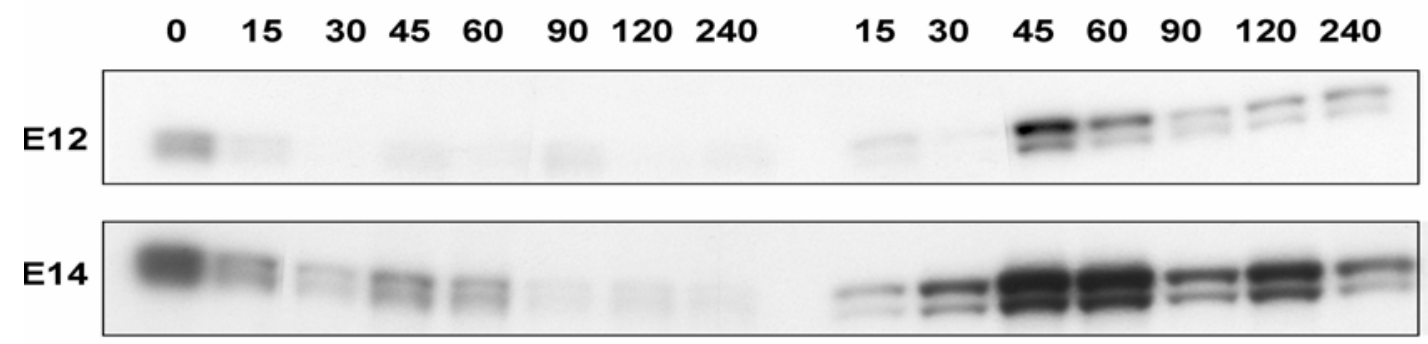

E16

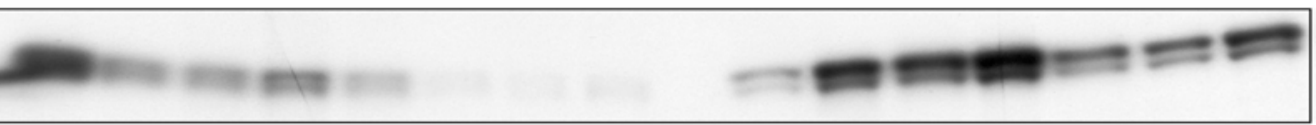

E18

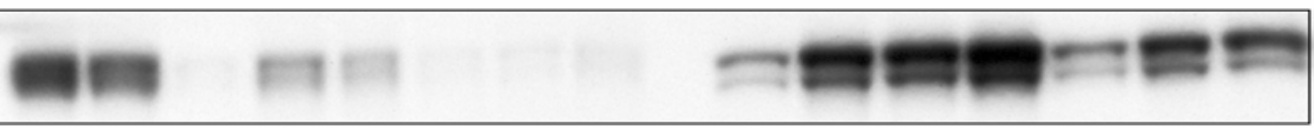

Figure 21: Kinetics for albumin (A) and AFP (B) in hepatoblasts during liver development estimated by pulse chase experiment. Hepatoblasts from developmental stages E12, E14, E16 and E18 as well as hepatocytes were kept in culture for two days, pulsed for $1 \mathrm{~h}$ with $100 \mu \mathrm{Ci} /$ well ${ }^{35} \mathrm{~S}$-methionine and then chased for 15, 30, 45, 60, 90, 120, $240 \mathrm{~min}$. Albumin and AFP were immunopecipitated with polyclonal anti-albumin and anti-AFP antibodies. The immunocomplexes were analysed by SDS-PAGE. Cell-lysates (Intracellular) and supernatants (extracellular) were used for immunoprecipitaion by taking into consideration samples with similar count. 
The kinetics for synthesis and secretion of albumin was measured in hepatoblasts isolated at 12, 14, 16 and 18 days of gestation after their cultivation for 2 and 9 days. The hepatoblasts were pulsed for $1 \mathrm{~h}$ with ${ }^{35} \mathrm{~S}$ metionine followed by incubation for a chase period of 2, 4 and 8 hours. The extent of albumin synthesis and secretion was determined from its immunoprecipitable radioactivity and expressed as ratio to count of total labelled proteins precipitated by trichloroacetic acid. Hepatoblasts from embryonic liver at E12 displayed an increase of albumin synthesis and secretion during the culture (Fig. 22). We observed a decrease of intracellular albumin after a chase period of $2 \mathrm{~h}$ followed by continuous secretion during a chase period of $4 \mathrm{~h}$ and a decrease of secreted albumin after a chase period of $8 \mathrm{~h}$. Interestinly, hepatoblasts isolated from developmental stages E14, E16 and E18 and cultured for 9 days could secrete completely albumin after a chase period of $4 \mathrm{~h}$. In contrast, hepatoblasts isolated from developmental stage E12 could secrete continuously albumin even after a chase period of $8 \mathrm{~h}$ (Fig. 22). 
A
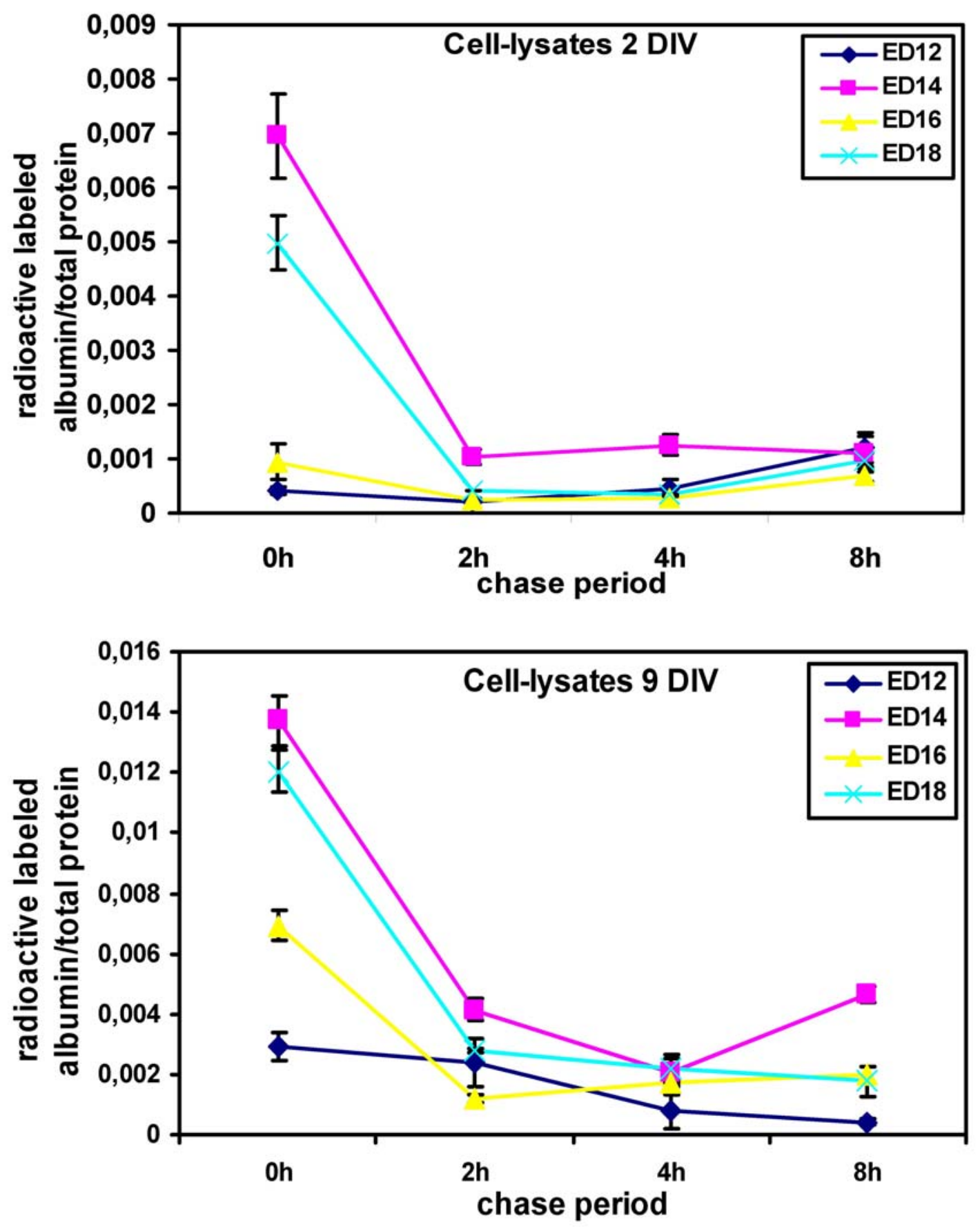
B
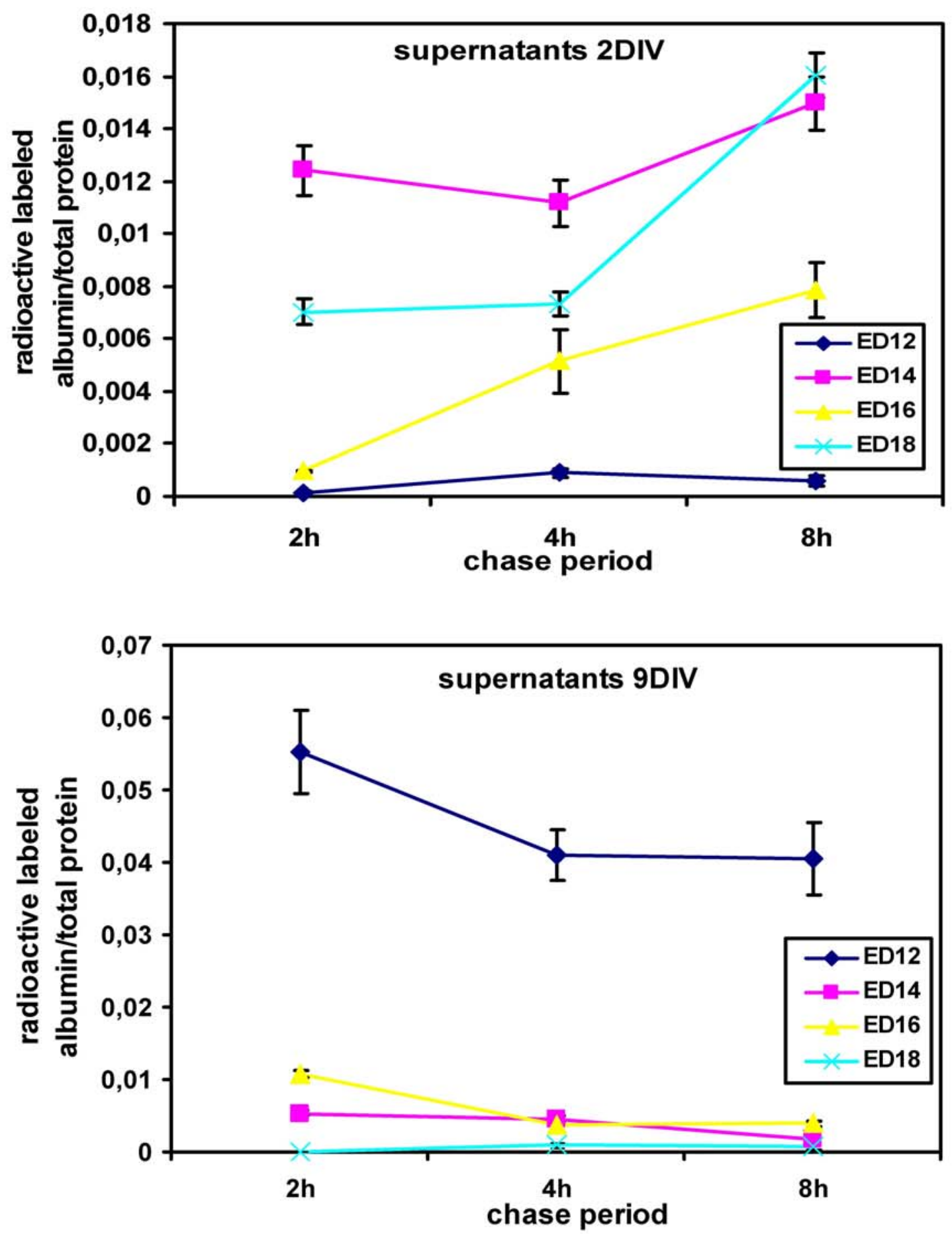
Figure 22 : Kinetics of albumin in hepatoblasts isolated at developmental stages E12, E14, E16 and E18 after 2 and 9 days in culture (DIV : days in vitro), measured by pulse chase experiment. Hepatoblasts were pulsed for $1 \mathrm{~h}$ with $100 \mu \mathrm{Ci} /$ well ${ }^{35} \mathrm{~S}$-methionine and then chased for $2 \mathrm{~h}, 4 \mathrm{~h}$ and $8 \mathrm{~h}$. Albumin was immunopecipitated from cell-lysates (A) and supernatants (B) which have a similar radioactive count measured after precipitation of total labelled proteins by trichloroacetic acid. The extent of albumin synthesis and secretion was determined from its immunoprecipitable radioactivity and expressed as ratio to count of total labeled proteins. Error bars represent S.E.M., $n=3$. The significance $(P<0.05)$ was analysed by ANOVA

\subsection{Expression of prox1, cytokeratin-7 and -19 during liver development.}

Prox1 is not expressed in CK-7 positive bile duct epithelial cells of the adult liver (Dudas et al. 2004). However, CK-7 is a late marker of the bile duct development, and it becomes detectable only in the foetal stage (Shiojiri et al. 1991). Prox1 is an early marker of hepatoblasts, which coexpress it with albumin and AFP (Dudas et al. 2004). Nevertheless, until now, it was not described how long Prox1 remains detectable in the nuclei of those hepatoblasts that differentiate toward bile duct cells. CK-19 was used to analyse embryonic and foetal hepatic cells, which may differentiate towards the biliary lineage. It was also not known whether CK-19 positive embryonic liver cells are always Prox1 positive and/or AFP positive.

At 14 and 16 days of gestation the majority of Prox1-positive cells in the developing rat liver showed cytokeratin-19 in their cytoplasm (Fig. 23. $(A, B)$ ), but cytokeratin-7 was not detected (Fig. 23 C). The coexpression of AFP and Prox1 in hepatoblasts isolated at E14 has been reported before (Dudas et al. 2004). At E16 some small CK-.19 positive, Prox1 negative and AFP negative cells were detected (Fig. $24(A, C)$ ). Prox1 was found mainly in AFP positive and CK-19 negative cells (Fig. 24 
B). At E18 the antigenic properties of Prox1 and CK-19 become clearly separated (Fig 26). We observed a colocalisation of Prox1 and CK-19 in some cells, they were only represented at $3.66 \%$ (by relating the Prox 1 positive/CK-19 positive cells to the whole population (Table 3 ), and the majority of the cells were either Prox1 positive or CK-19 positive (Fig. 25A). Co-localisation of CK-19 with AFP at E 18 was found only in $3.8 \%$ of the whole cell population (Fig. 26) (Table 3). CK-7 was first detected at E18 in Prox1 negative cells (Fig. 25B). The co-localisation of prox1 and CK-19 at embryonic stages (E14 and E16) and their expression separately in different cell-types at foetal stage (E18) was confirmed in cultured hepatoblasts. At the foetal stage (E18), we identified three cell populations, Prox1 positive/CK-19 positive cells, Prox1 negative/CK-19 positive cells and Prox1 positive/ CK19 negative cells (Fig. 27).

Cell nuclear Prox1 immunostaining co-localisation with the cytoplasmatic reaction of HepPar1 and the connexin 32-positive gap junctions indicate that hepatoblasts from E18 display a mature hepatocyte phenotype (Fig. $25(C, D))$. Prox1 remained a stable cell nuclear marker in adult hepatocytes, and was absent in bile ducts, while the latter were CK-19 or CK-7 positive (Fig. $28(A, B)$ ). The hepatocyte immunophenotype was confirmed by positive reactions with anti-HepPar1 and anti-connexin 32 . Prox1 was detected in parenchymal cells connected by connexin 32 containing gap junctions, while sinusoidal cell nuclei were negative (Fig.28 $(C, D, E))$ 

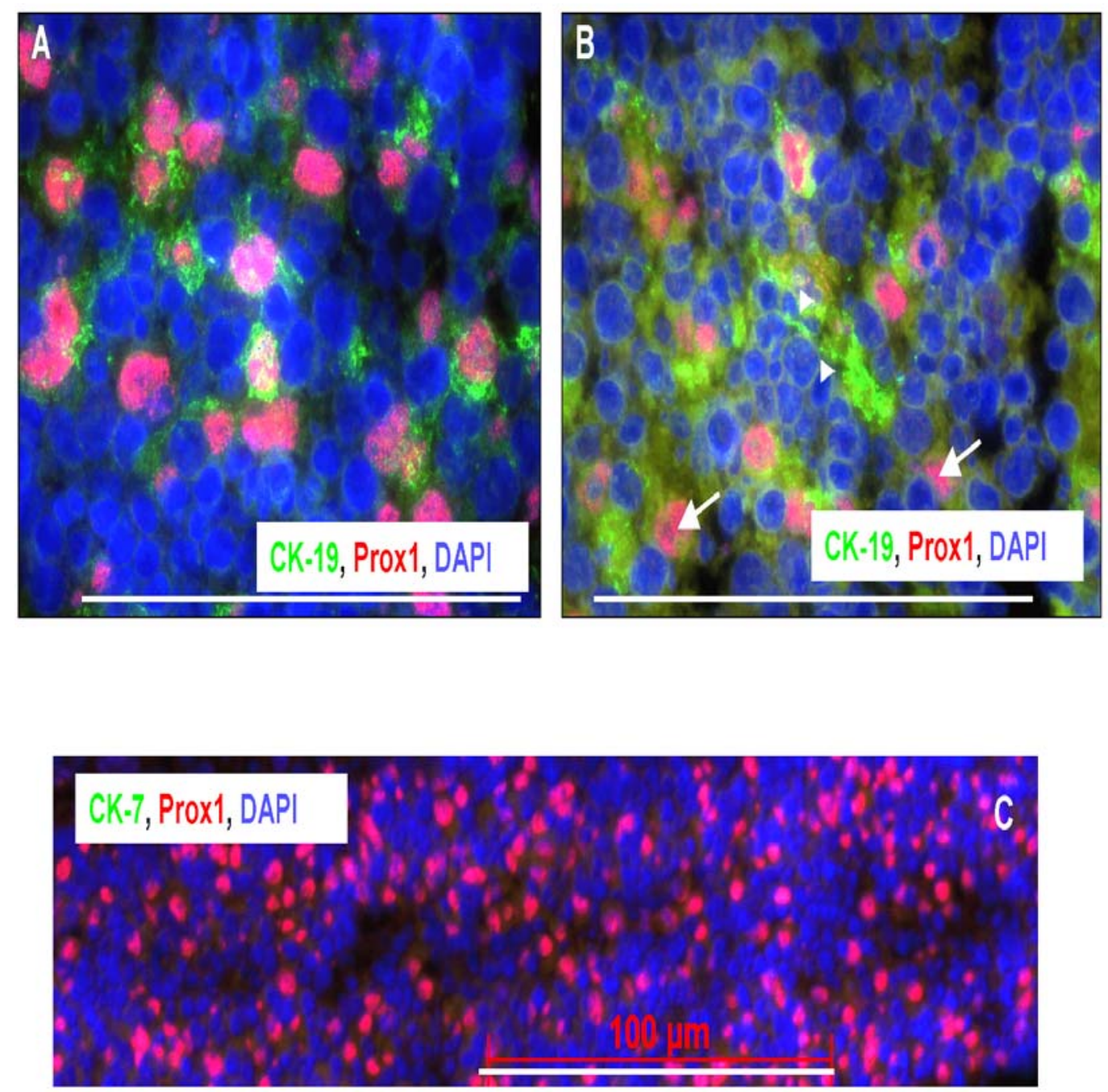

Figure 23: Prox1-, CK-19-, and CK-7-specifc immunohistochemical reactions in embryonic rat liver at E14 and E16. (A) At E14, cells with Prox1(red) positive nuclei show CK-19 (green) in the cytoplasm (Bar $=50 \mu \mathrm{m})$. (B) At E16, the Prox1-positive hepatoblasts partly show CK-19 staining, although in several cells the stainings are separated (showing CK-19 without Prox1 (arrowheads) or Prox1 without CK-19 (arrows) (Bar $=50 \mu \mathrm{m}$ ). (C) Liver section at E16 with immunofluorescent staining for CK-7 and Prox1. CK-7 (green) positive reaction is still not detected. Counterstaining of nuclei was performed with DAPI (blue) $($ Bar $=100 \mu \mathrm{m})$. 

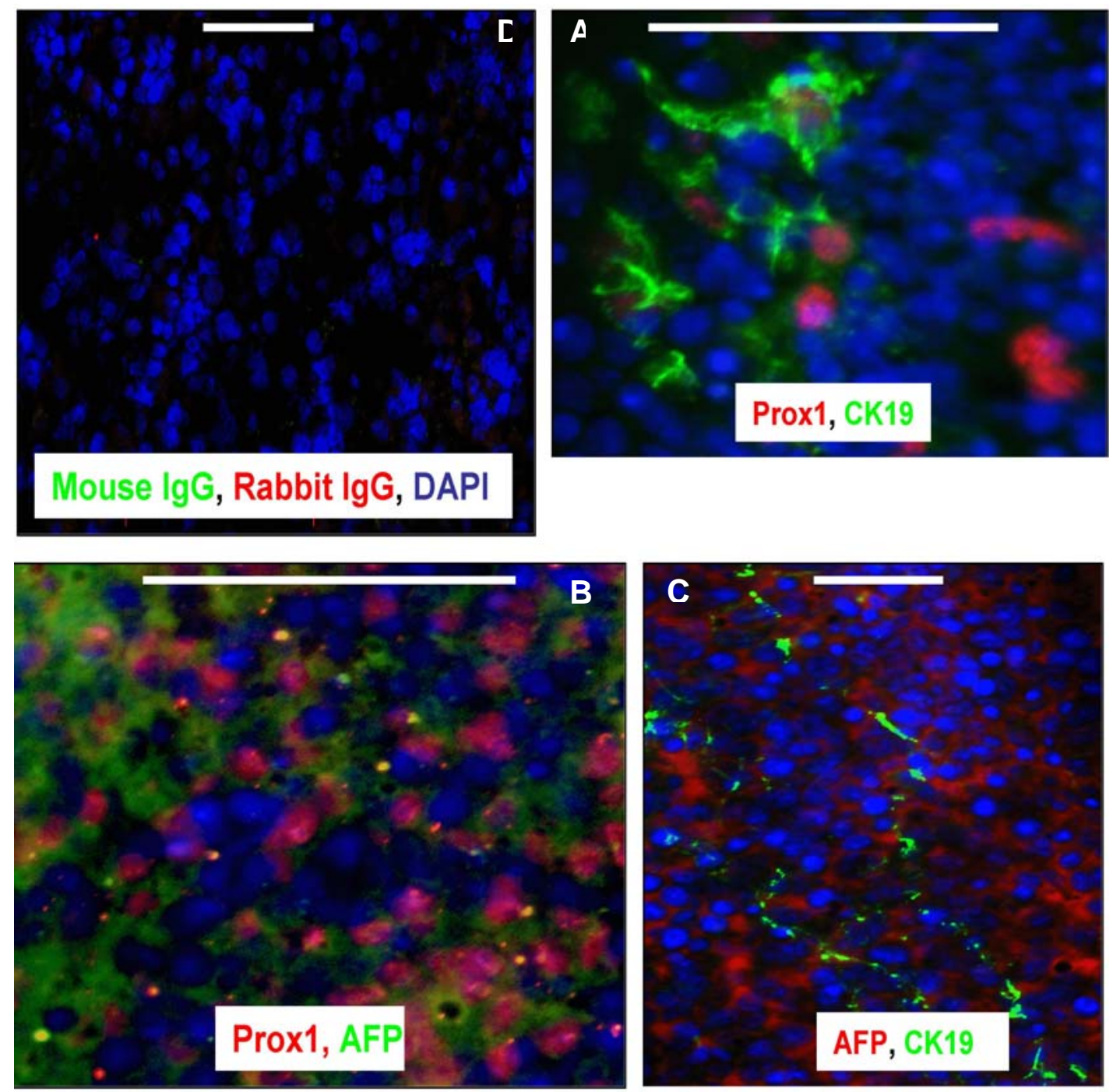

Figure 24: Immunohistochemical reactions with anti-Prox1 (red), anti-AFP (red and green) and anti-CK-19 (green) antibodies. counterstaining of nuclei with DAPI (blue). Prox1 negative/CK-19 positive(A) and Prox1 positive/AFP positive $(\mathbf{B})$ cells are detected $($ Bar $=50 \mu \mathrm{m})$, the small CK-19 positive cells are AFP negative $(\mathbf{C})($ Bar $=100 \mu \mathrm{m})$. In negative control (performed with rabbit and mouse IgG, and secondary antibodies) of liver section at ED 16 no red or green reactions were observed $($ Bar $=100 \mu \mathrm{m})$. 

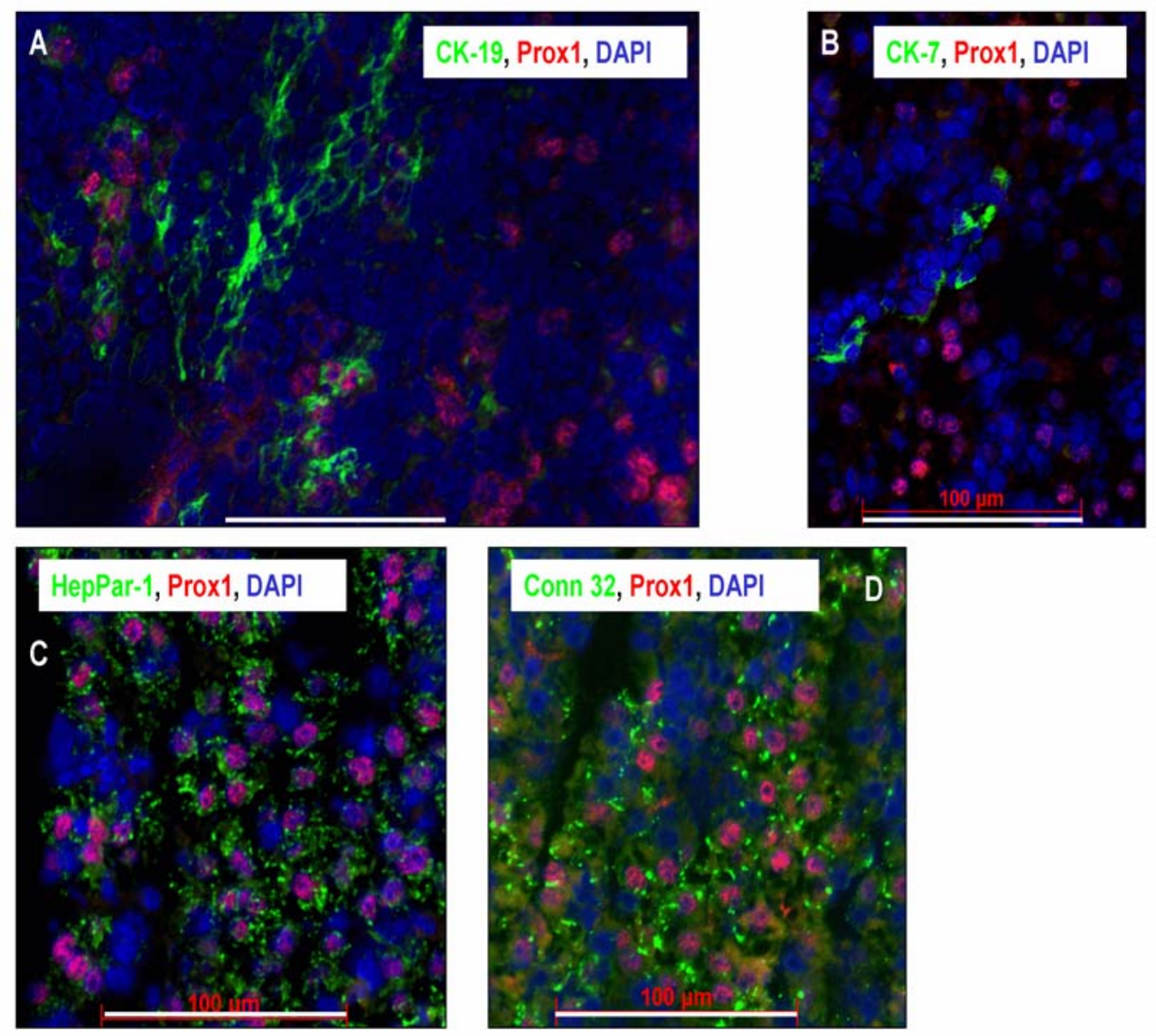

Figure 25: Reactions with anti-Prox1 (red) anti-CK-19 (green), anti-CK-7 (green), anti-HepPar-1 (green) and anti-connexin 32 (green) antibodies, counterstaining of nuclei with DAPI (blue) in the liver of rat embryos at 18 days of gestation. (A) Section of rat liver show positivity for CK-19 and Prox1. The Prox1 and CK-19 reactions are separated, but double positive cells are also present (Bar $=100$ $\mu \mathrm{m})$. (B) Section of rat liver show positivity for CK-7 and Prox1. The Prox1positive hepatoblasts and the CK-7 positive bile duct epithelial cells are separated $($ Bar $=100 \mu \mathrm{m})$. (C) Section of rat liver show immunofluorescent staining for HepPar1 and Prox1 (Bar $=100 \mu \mathrm{m})$. (D) Section of rat liver show a positive staining for connexin 32 and Prox1 (Bar $=100 \mu \mathrm{m})$. Cell nuclear Prox1 immunostaining co-localises with the cytoplasmatic reaction of HepPar1 and the connexin 32-positive gap junctions. 

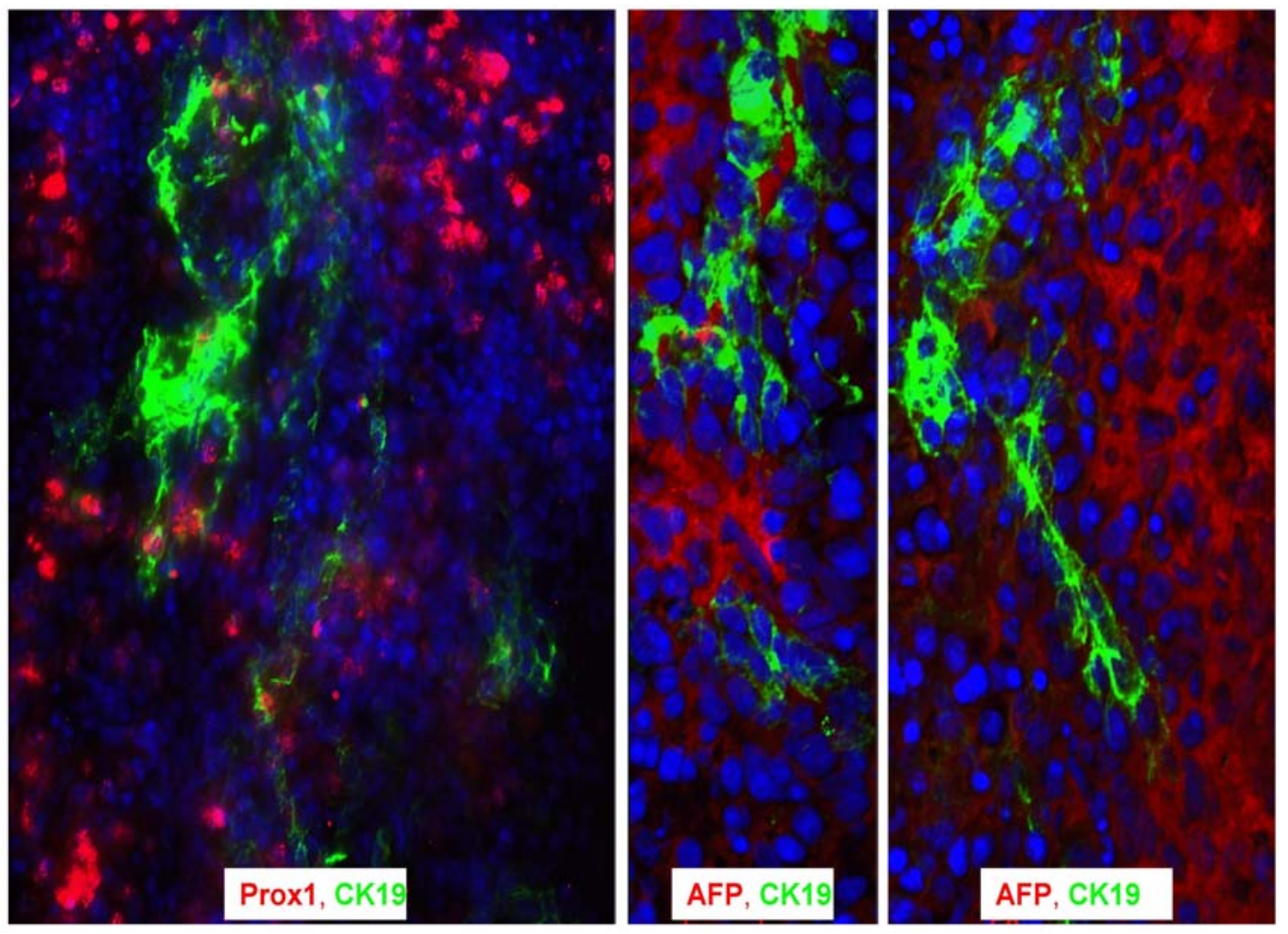

Figure 26: Prox1-, CK-19-, AFP-specific immunohistochemical reactions in embryonic rat liver at E18. CK-19 positive cells (green) are mainly Prox1 negative $(r e d)(B a r=50 \mu \mathrm{m})$, the CK-19 positive cells are AFP negative (red) are also detected in different cells $($ Bar $=50 \mu \mathrm{m})$ 

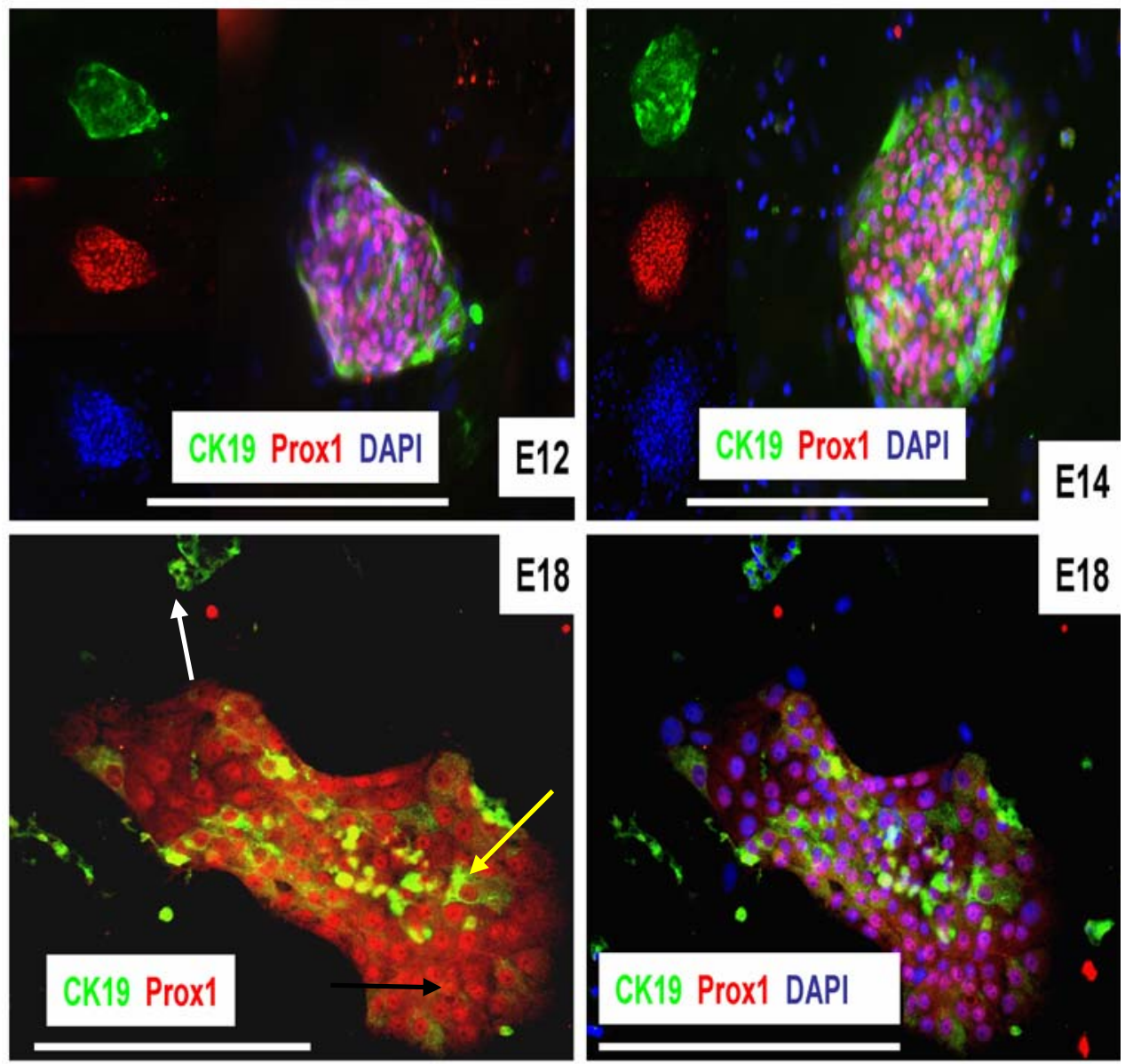

Figure 27: Prox1-, CK-19-specific immunocytochemical reactions in cultured hepatoblasts generated from developmental stages E12, E14 and E18. Hepatoblasts from E12 and E14 are Prox1 (red) and CK-19 (green) positive. At E18 three cell populations are observed. Prox1 and CK-19 positive cells (yellow arrows), Prox1 positivel CK-19 negative cells (black arrows) and CK-19 positive/Prox1 negative (white arrows). Counterstaining of nuclei was performed with DAPI (blue) $($ Bar $=100 \mu \mathrm{m})$. 

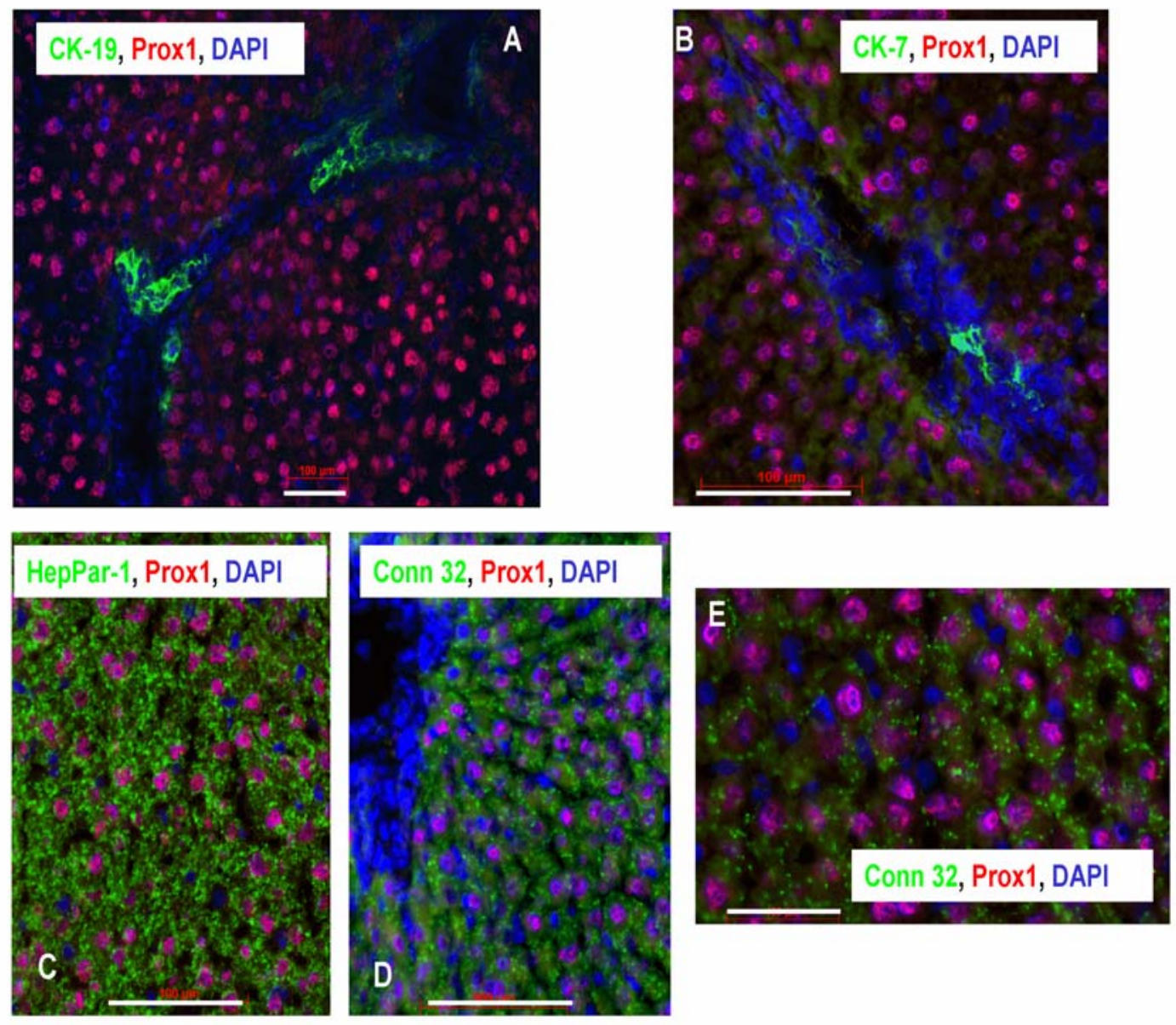

Figure 28: Reactions with anti-Prox1 (red) anti-CK-19 (green), anti-CK-7 (green), anti-HepPar-1 (green) and anti-connexin 32 (green) antibodies, counterstaining of nuclei with DAPI (blue) in adult rat liver. (A) Immunofluorescent staining for CK-19 and Prox1. The Prox1 and CK19 reactions are separated (Bar $=50 \mu \mathrm{m})$. (B) Immunofluorescent staining for CK-7 and Prox1. The Prox1 and CK-7 reactions are separated (Bar $=50 \mu \mathrm{m})$. (C) Indirect immunofluorescent staining for HepPar1 and Prox1 (Bar $=50 \mu \mathrm{m})$. (D-E) Immunofluorescent stainings for connexin 32 and Prox1. Cell nuclear Prox1 immunostainings co-localise with the cytoplasmatic reaction of HepPar1 and the connexin 32-positive gap junctions (Bar $=50 \mu \mathrm{m})$. 


\begin{tabular}{cccccc}
\hline & ED14 & ED 18 & & ED14 & ED 18 \\
\hline Prox1 $1^{+} / \mathrm{CK} 19^{+}:$ & $28.8 \pm 4.13 \%$ & $3.66 \pm 0.04 \%$ & $\mathrm{AFP}^{+} / \mathrm{CK} 19^{+}:$ & $36.69 \pm 5.79 \%$ & $3.8 \pm 0.09 \%$ \\
& & & & \\
Prox1 $1^{+} / \mathrm{CK} 19:$ & $8.8 \pm 0.79 \%$ & $31.53 \pm 13.31 \%$ & $\mathrm{AFP}^{+} / \mathrm{CK} 19:$ & $12.84 \pm 2.10 \%$ & $36.53 \pm 3.28 \%$ \\
& & & & \\
Prox1 $/$ CK19 $:$ & $5.2 \pm 1.38 \%$ & $13.51 \pm 5.90 \%$ & $\mathrm{AFP}^{-} / \mathrm{CK} 19^{+}:$ & $8.92 \% \pm 3.03 \%$ & $14.92 \pm 1.67 \%$ \\
\hline
\end{tabular}

Table 3: Quantitative comparison of Prox1- CK-19- and AFP-specific immunohistochemical reactions at embryonic (E14) and foetal stages (E18) of rat liver development. Single- and double-positive cell counts were related to all cell nuclei in the image and expressed in average \%+ standard error of measurement (SEM) \%. In all quantitative immunohistochemical analysis 15 random taken samples from the investigated sections were analyzed

\subsection{Effect of interleukin-6 on synthesis and secretion of albumin and AFP}

The effect of interleukin-6 (IL-6) on synthesis (intracellular) and secretion (extracellular) of albumin and AFP was investigated during liver development. Hepatoblasts derived from 12, 14 and 18 days of gestation were stimulated with $100 \mathrm{ng} / \mathrm{ml} \mathrm{IL-6}$ and radioactive labelled with ${ }^{35} \mathrm{~S}$ methionine for $12 \mathrm{~h}$. It has been observed that IL-6 has a positive effect on hepatoblasts derived from 14 and 18 days of gestation, whereas synthesis and secretion of albumin and AFP were suppressed. A slight effect of IL-6 on synthesis and secretion of AFP and a high effect of IL-6 on synthesis of albumin was observed in hepatoblasts from 12 days of gestation (Fig. 29A). This finding was confirmed by measurement of mRNA-expression levels of interleukin- 6 receptor (IL-6 rec) in hepatoblasts using real time RT-PCR experiment. Hepatoblasts from 14 and 18 days of gestation displayed a high expression of interleukin-6 receptor (IL-6 rec) mRNA than hepatoblasts from E12 (Fig. 29B). 
A

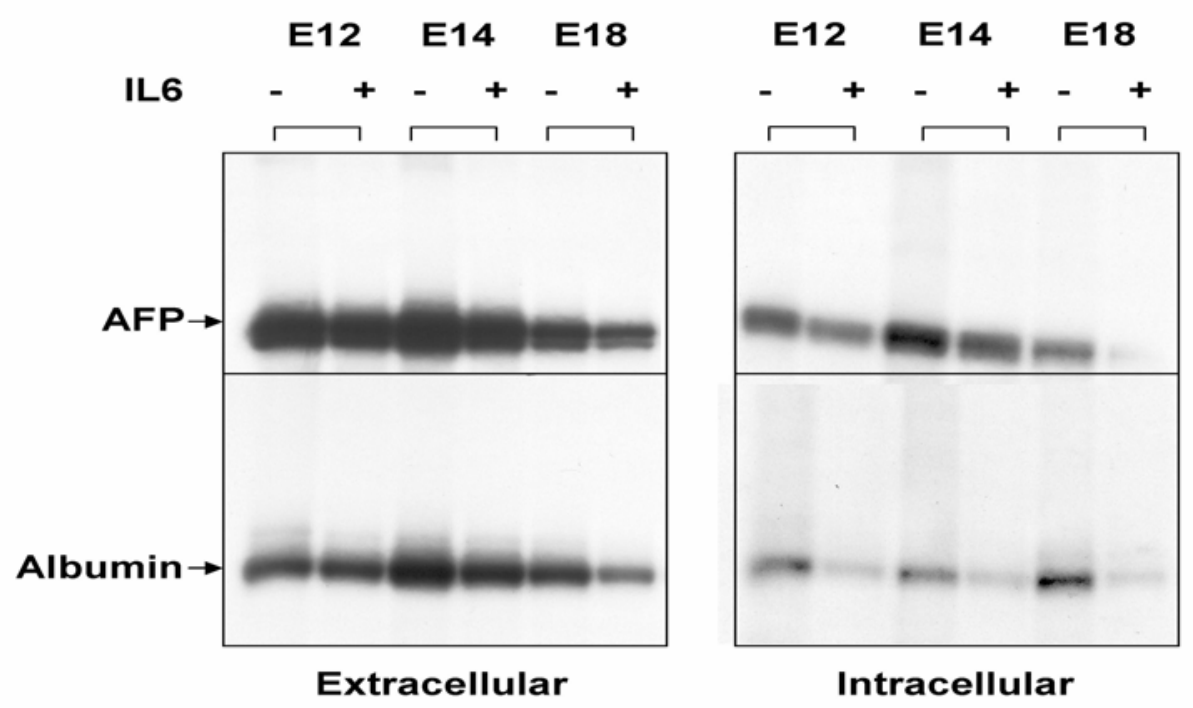

B

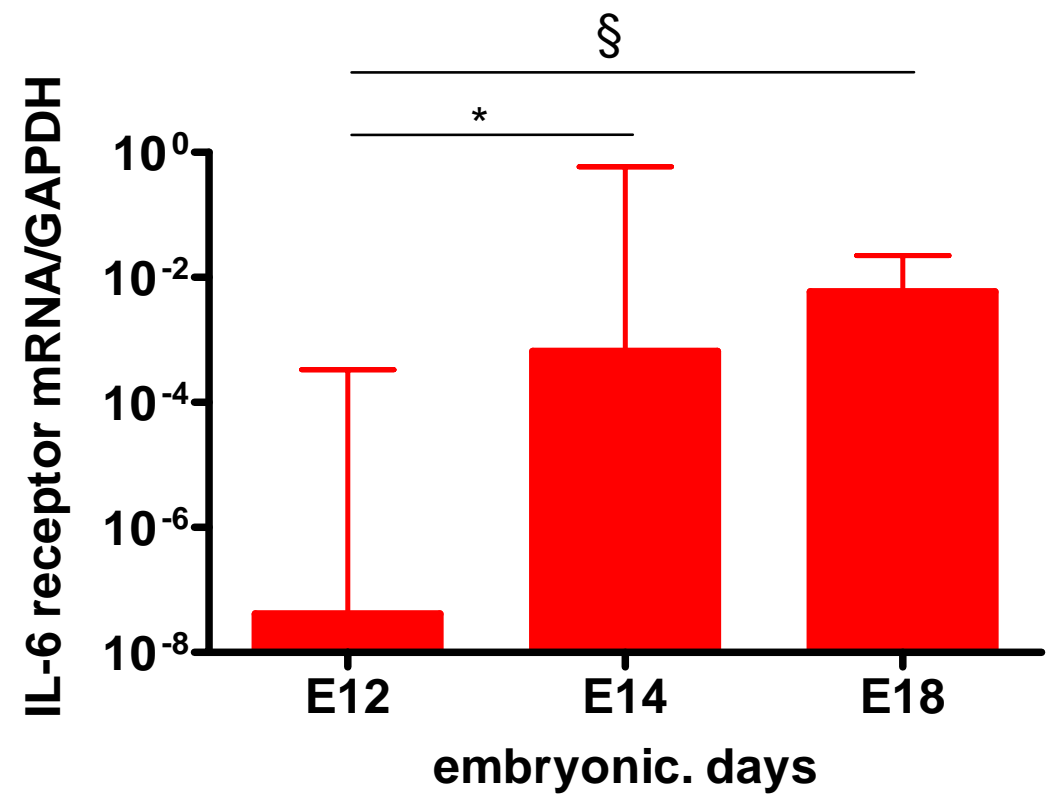

Figure 29: (A) Effect of interleukin-6 (IL-6) on synthesis and secretion of albumin and AFP in hepatoblasts at developmental stage E12, E14, E18. The cells were stimulated with $100 \mathrm{ng} / \mathrm{ml}$ interleukin-6 and radioactive labelled with $100 \mu \mathrm{Ci}^{35} \mathrm{~S}$ methionine simultaneously overnight. Cell-lysates (intracellular) and supernatants (extracellular) with similar count were used for immunopecipitation of albumin and AFP. (B) Real time PCR analysis of interleukin-6 receptor mRNA-expression in unstimulated hepatoblasts. The expression was normalized with the endogenous control of GAPDH. Error bars represent S.E.M, $n=3$. Statistically significant difference $\left({ }^{\star} \mathrm{P}<0.05 ; \S<0.05\right)$ analysed by student's $t$-test 


\subsection{Characterisation of hepatoblasts after different passages}

Hepatoblasts derived from 12 and 14 days of gestation were passaged three times and analysed with a view to find out if hepatoblasts could maintain hepatic phenotype after the passage. Albumin and AFP geneexpression was measured at each passage by using real time PCR and radioactive biosynthetic labelling experiment. We found that after the first passage, hepatoblasts could express, synthesize and secrete albumin and AFP. During the second passage albumin and AFP could not be detected, (Fig. 30 and 31).
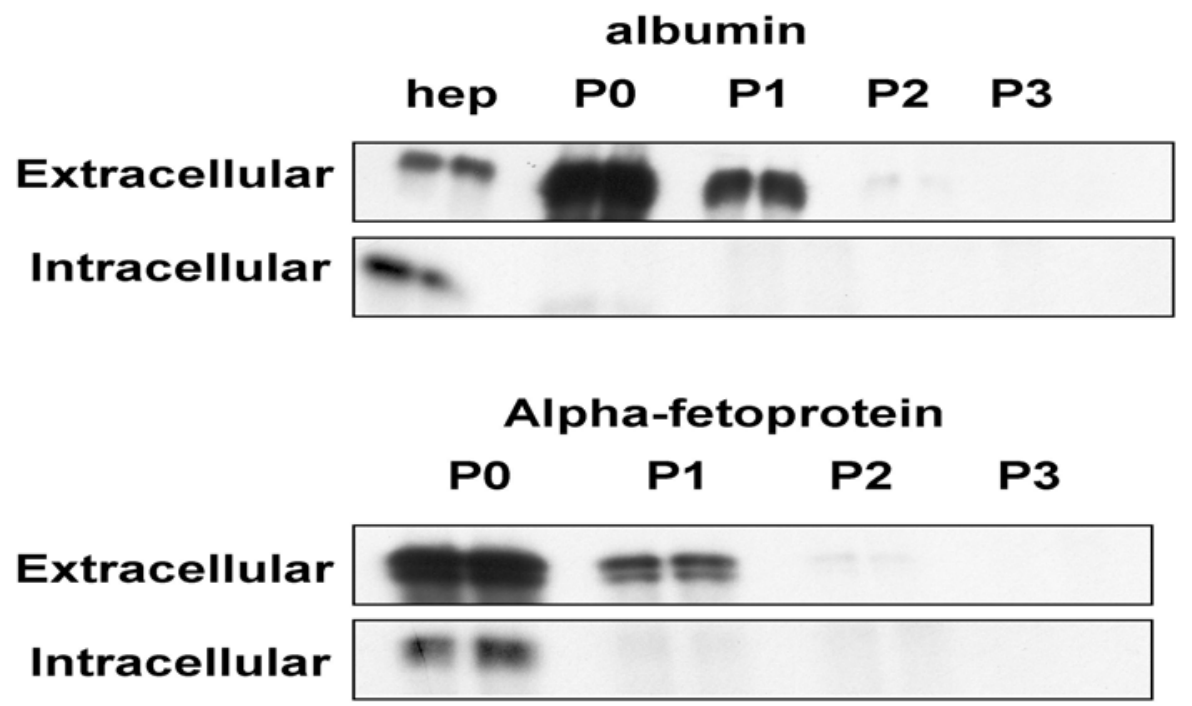

Figure 30: Synthesis and secretion of Albumin and AFP in passaged hepatoblasts derived from 12 and 14 days of gestation. At each passage, cells were labelled with $100 \mu \mathrm{Ci}{ }^{35} \mathrm{~S}$-methionine per well. Albumin and AFP were immunopecipitated from $100 \mu \mathrm{l}$ cell-lysates (Intracellular) and supernatants (extracellular). As positive control, $25 \mu \mathrm{l}$ supernatant and cell-lysates of labelled hepatocytes were used. 
A
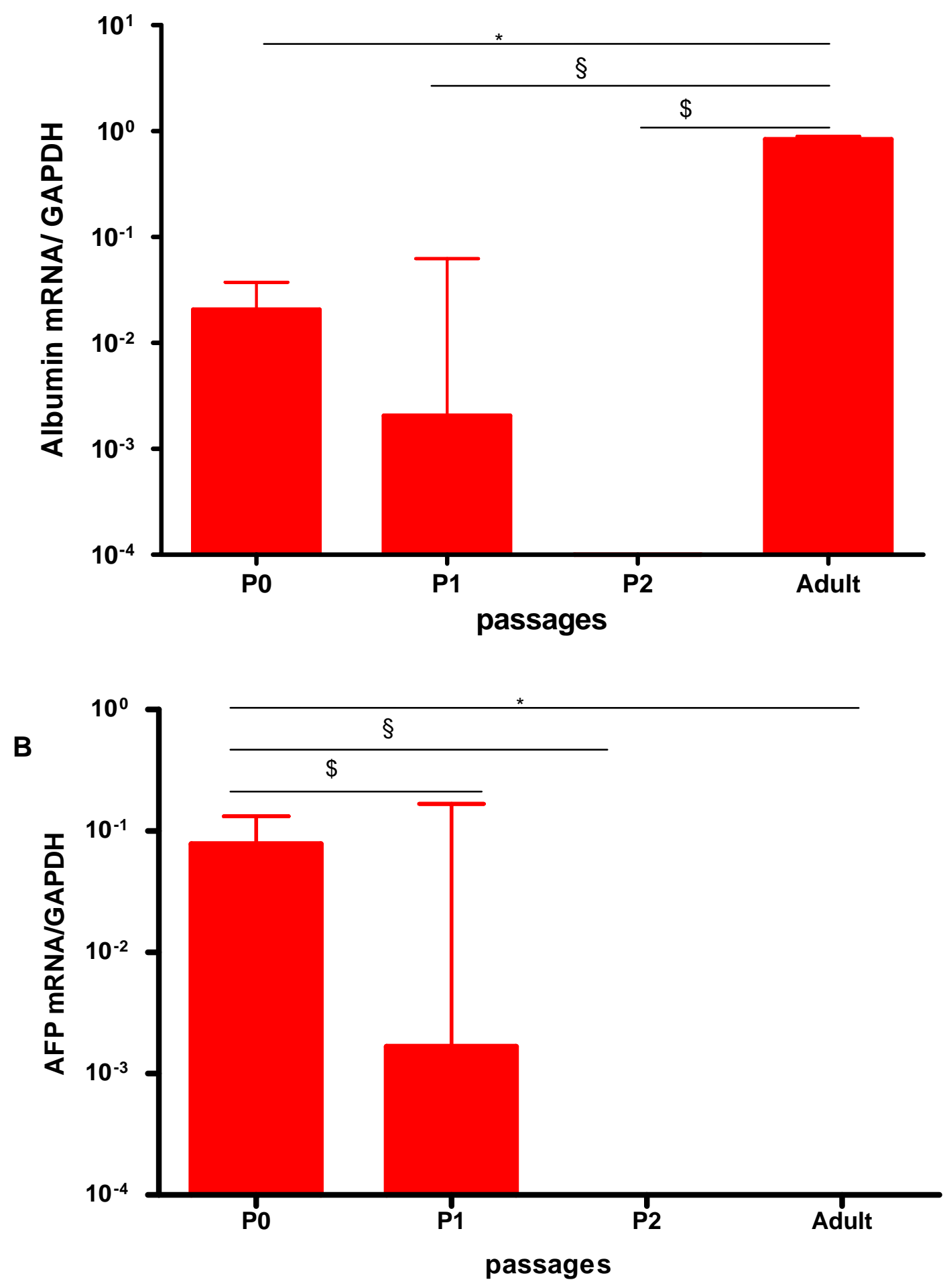

Figure 31: Real-time PCR analysis of albumin (A) and AFP (B) mRNAexpression after passage of hepatoblasts. The expression was normalized with the endogenous control of GAPDH. Error bars represent S.E.M, $n=3$. Statistically significant difference ( ${ }^{\star} \mathrm{P}<0.05 ; \S<0.05 ; \$<0.05$.) analysed by student's $t$-test. 


\subsection{Changes in gene expression of hematopoietic regulatory cytokines during liver development}

In adult bone marrow, the cytokine controlled hematopoiesis were expressed by nonhematopoietic stromal cells, including fat cells, endothelial cells, fibroblasts and macrophages. In case of inflammation, the cytokines could be synthesized by activated $T$ lymphocyte and macrophage. The embryonic/foetal liver is a mosaic of cells consisting of hepatoblasts, which are mainly represented, hematopoietic stem cells, endothelial cells and fibroblasts. Gene expression of granulocytemacrophage colony-stimulating factor (GM-CSF), granulocyte colonystimulating factor (G-CSF), stem cell factor (SCF) and Erythropoietin (Epo) was examined in whole liver (in vivo) and cultured embryonic hepatic cells (in vitro) during liver development.

At 14 days of gestation, in whole liver (in vivo) a strong and statistically significant mRNA expression of the cytokines GM-CSF and Epo was observed (Fig. 32 .A and D). G-CSF was expressed only in cultured hepatic cells (in vitro). In contrast, Epo was expressed only in whole liver (in vivo) (Fig. $32 \mathrm{D}$ ). G-CSF mRNA was high expressed at 14 days of gestation. It underwent a moderate expression at 16 days of gestation and at new born state (Fig. $32 \mathrm{~B}$ ). It was observed that the embryonic liver expressed high amount of SCF at 12 day of gestation and slight amount at 14 and 16 days of gestation. During the foetal and postnatal life SCF was down-regulated (Fig. 32 C). 
A

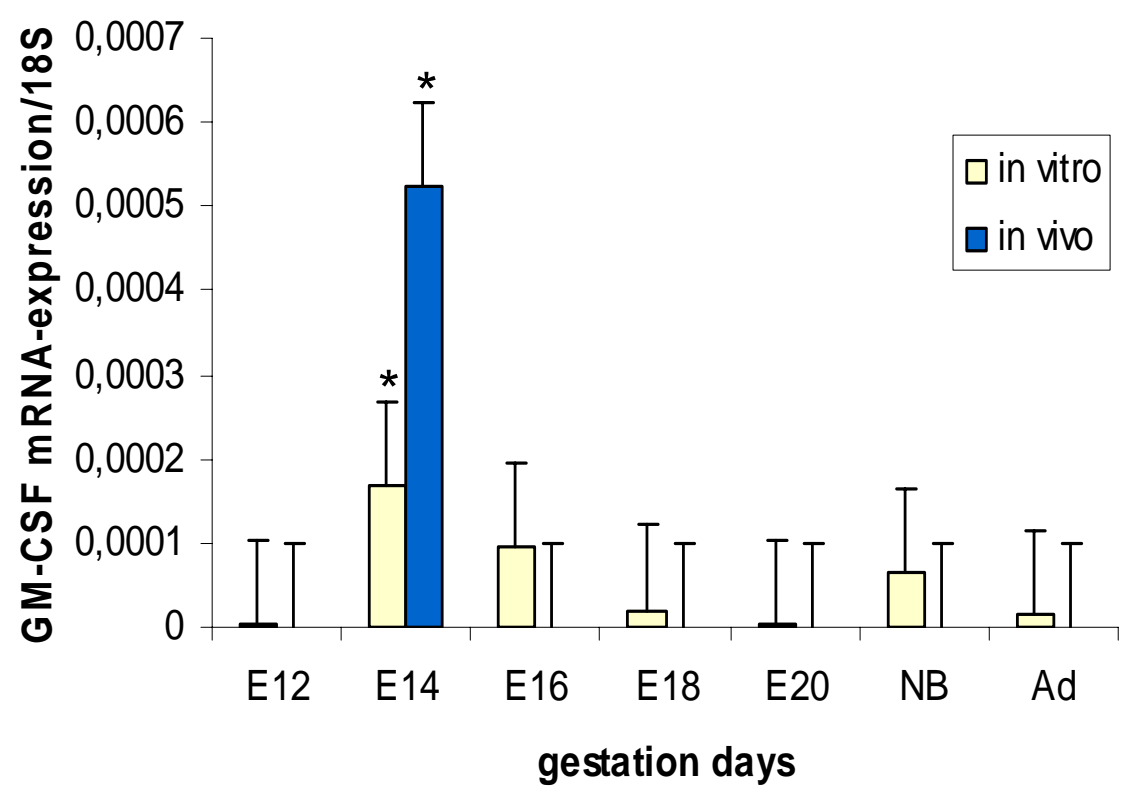

B

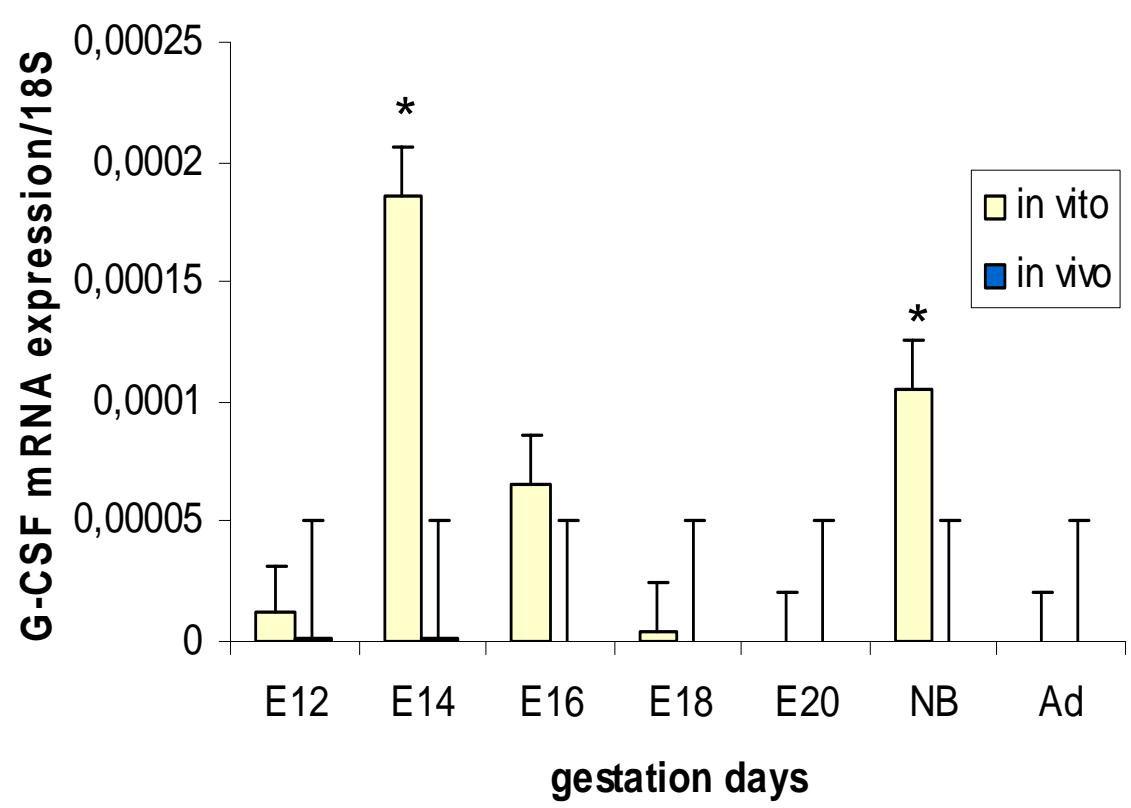


C

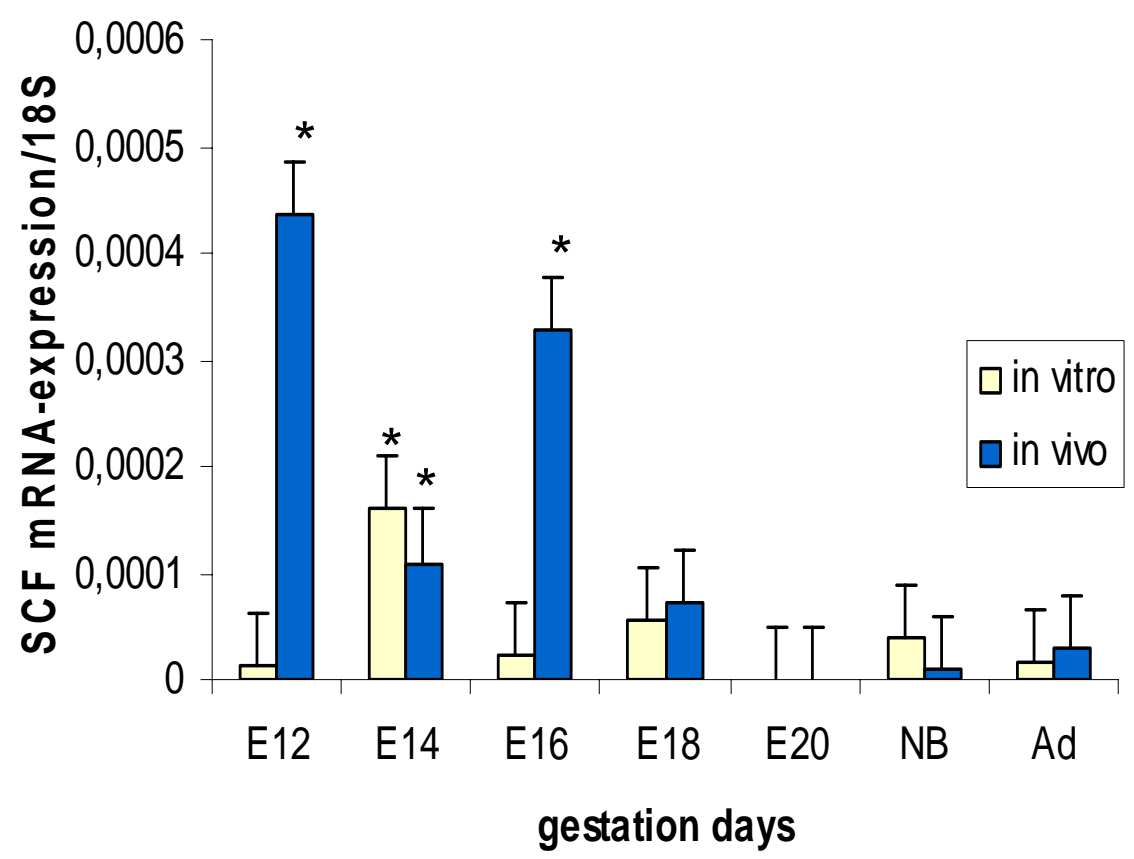

D

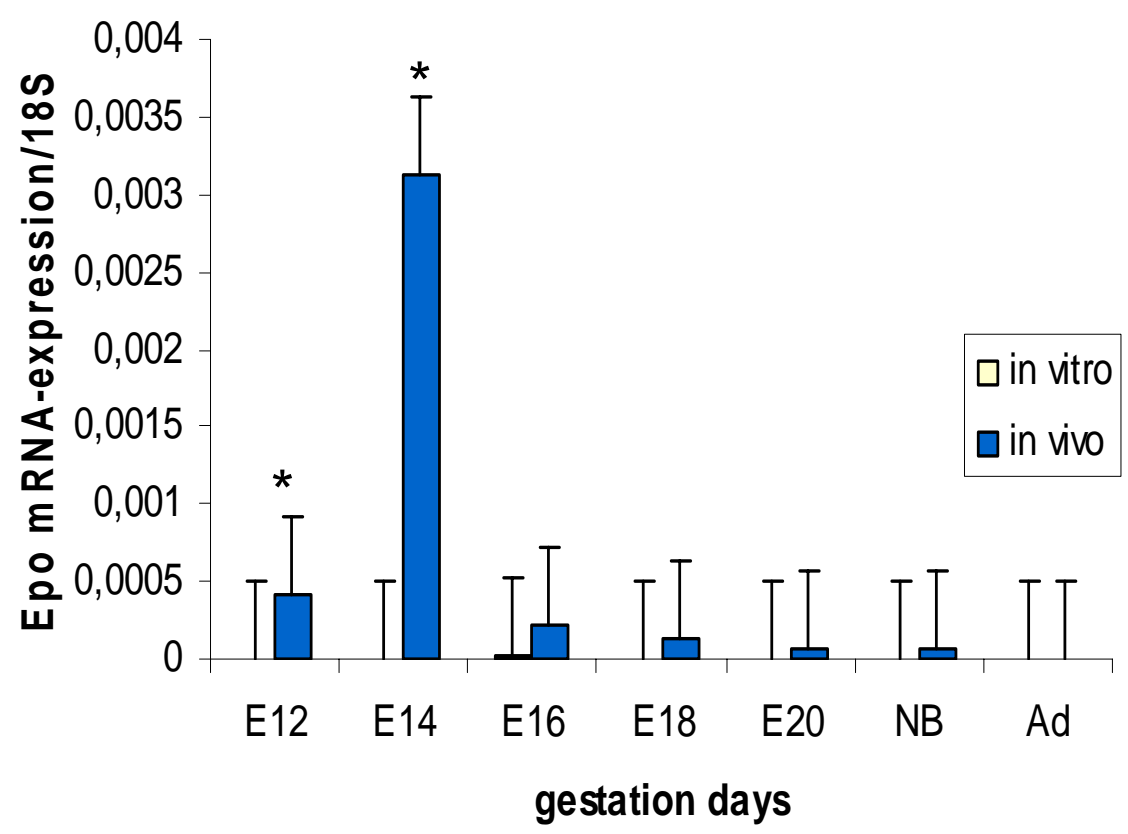


Figure 32: Real-time-PCR -analysis of mRNA from whole liver (in vivo) and cultured hepatic cells (in vitro). Livers were obtained and the hepatic were cultured at different developmental stage as described in materials and methods. Fold change of granulocyte-macrophage colony-stimulating factor (GM-CSF) (A), granulocyte colony-stimulating factor (G-CSF) (B), stem cell factor (SCF) (C) and Erythropoietin (Epo) (D) gene-expression during liver development. Values represent the amount of target mRNA compared to $18 \mathrm{~S}$ ribosomal mRNA $\left({ }^{*} \mathrm{P}<0.05\right.$. Error bars represent S.E.M., $\mathrm{n}=3$ )

\begin{tabular}{|c|c|c|c|c|c|c|c|}
\hline & \multicolumn{7}{|c|}{ Embryonic developmental stage } \\
\hline Genes & E12 & E14 & E16 & E18 & E20 & New born & adult \\
\hline GM-CSF in vivo & - & +++ & - & - & - & - & - \\
\hline GM-CSF in vitro & - & + & + & $+/-$ & - & $+/-$ & - \\
\hline G-CSF in vivo & - & - & - & - & - & - & - \\
\hline G-CSF in vitro & $+/-$ & +++ & + & $+/-$ & - & ++ & - \\
\hline SCF in vivo & +++ & + & ++ & + & - & $+/-$ & $+/-$ \\
\hline SCF in vitro & $+/-$ & ++ & $+/-$ & $+/-$ & - & $+/-$ & $+/-$ \\
\hline Epo in vivo & + & +++ & $+/-$ & $+/-$ & $+/-$ & $+/-$ & $+/-$ \\
\hline Epo in vitro & - & - & - & - & - & - & - \\
\hline
\end{tabular}

+++ : high expression

++ : moderate expression

+ : low expression

$-/+$ : very low expression

- : no expression

Table 4: Summary of the mRNA expression levels of cytokines GM-CSF, G-CSF, SCF and Erythropoietin (Epo) in whole liver (in vivo) and cultured hepatoblasts (in vitro) during liver development. 


\section{DISCUSSION}

\subsection{Albumin and AFP gene expression at the time of hepatic specification (E10)}

The results presented in section 3.2 describe the expression of albumin and AFP mRNA in the ventral foregut region of E10 rat embryos (0-4 somites) as well as in explanted cells generated from ventral foregut endoderm. Previous studies using rat embryos claimed that AFP could be expressed one day before albumin expression (Shiojiri et al., 1991b; Muglia and Locker, 1984)). Muglia and Locker, (1984) used a blothybridization followed by densitometric analysis at several exposures. They reported that albumin and AFP mRNA were not detectable in the 10day RNA preparation taken from the foregut region, even after long exposure times. In 12-day liver RNA, the expression of both genes was observed. Using In situ histological experiments neither albumin mRNA nor AFP mRNA could be detected at E9.5-E10. At E10.5 in the ventral endoderm AFP mRNA was clearly detected one day before its coding protein (E11.5). At 11.5 days of gestation albumin mRNA was identified, but its protein appeared one day later (E12.5) (Shiojiri et al., 1991a). This finding is consistent with the studies using 4 to 6 somites mice embryos (E8-E9.5) (corresponding to E9.5-E11 in the rat). It has been shown that AFP mRNA is detected by in situ hybridization performed on whole embryos or in explanted cells derived from ventral region of embryos (Gualdi et al., 1996) (Lee et al., 2005). RT-PCR experiment provided a possibility to detect AFP mRNA in the ventral and dorsal endoderm. Albumin mRNA was detected only in explants generated from ventral endoderm containing presumptive cardiac mesoderm or after stimulation 
with different types and different concentration of FGFs (Deutsch et al., 2001; Jung et al., 1999; Gualdi et al., 1996; Micsenyi et al., 2004; Serls et al., 2005). In this study, we demonstrated by real time PCR that unstimulated explanted cells derived from ventral foregut region could express albumin mRNA.

Previous histological studies have not examined the synthesis and secretion of albumin and AFP in viable endodermal cells. Previously albumin-expression at protein level was detected by immunofluorescence analysis in sectioned mouse embryos from 9-10 somites stages as well as in single cell cytospin slides generated from dissected ventral foregut endoderm at 8, 10 and 14 somites (Serls et al., 2005). In this study we established, for the first time, a reliable method, using a sensitive radioactive biosynthetic labelling, for analysing the albumin and AFP synthesis and secretion capacity of endodermal cells generated from the ventral foregut region. Our results suggest that the whole program controlling the regulation of gene expression, synthesis and secretion of albumin and AFP already act at the earliest developmental stage, when hepatic specification of endoderm cells begins.

\subsection{Characterisation of endodermal cells}

The endodermal germ layer gives rise to a number of different organs including the liver. The characterization of endodermal cell populations, which develop into hepatic buds, has not been previously thoroughly investigated. The present study shows that explanted endodermal cells from ventral endoderm could express HNF4-alpha, Prox1, beta-catenin, BMP-4, Foxa-2, and GATA-4. These factors play crucial role in 
hepatogenesis. GATA-4 homozygous null mice display defects in ventral foregut development (Molkentin et al., 1997) and GATA-4 is required for endoderm development (Narita et al., 1997; Bossard and Zaret, 1998). BMP-4 signalling transmitted from mesenchymal cells allow the endoderm to induce liver gene expression in response to FGFs (Rossi et al., 2001). Prox1 is expressed in the endoderm (Burke and Oliver, 2002) and its activity is essential for liver development, due to its role in regulation of genes that control proliferation and migration of hepatoblasts (SosaPineda et al., 2000). HNF4-alpha is one of the earliest primary endoderm markers, its expression is restricted during liver development (Duncan et al., 1994). In the adult organism HNF4-alpha is expressed in the liver and is capable to activate the expression of numerous hepato-specific genes (Tian and Schibler, 1991). In this study we demonstrated that HNF4-alpha is expressed by in culture clustered endodermal cells derived from ventral foregut region, which could be the expressing cells for albumin and AFP. Foxa-2 (HNF3beta) is first detected on the seventh day of murine gestation (according to 8.5 day in the rat) in the primitive streak and node (Ang et al., 1993). It has been shown that the Foxa-transcription factors participate in alteration of chromatin structures during activation of liverspecific target genes (Zaret, 2002). Beta-catenin is one of the key proteins in the wnt/beta-catenin pathway. It has been reported that this pathway plays a crucial role during liver development and could activate target gene expression that controls proliferation, adhesion, polarity, apoptosis and lineage specification (Monga et al., 2003); (Willert and Nusse, 1998). The origin, localisation and migration of endodermal cells were examined by endoderm fate maps study in mice embryos from early stage (1 to 6 
somites). It has been demonstrated that a cell population at the ventral midline of the endoderm lip (VMEL) express a liver specific gene HNF4alpha (Tremblay and Zaret, 2005).

\subsection{Cellular analysis during liver development}

At 12 days of gestation, when the embryonic liver is formed, albumin and AFP genes are mainly expressed by hepatoblasts. In this study we demonstrated that during embryonic and foetal stages about $50 \%$ of liver cells are engaged in both albumin and AFP gene expression. In addition the ratio of albumin and AFP producing cells to proliferating cells increase during embryonic stage. At 18 days of gestation the ratio of albumin and AFP producing cells to proliferating cells reaches its maximum. From this stage up to birth, we observed an increase of the number of albumin producing cells. After 18 days of gestation the number of proliferating cells decrease and hepatoblasts produce more albumin than AFP. The volume of the liver expands 84-fold during liver development, and this correspond to 8 doubling of hepatoblasts (Greengard et al., 1972); (Vassy et al., 1988). It is especially noteworthy that, while an increase of liver size and an increase of the ratio of albumin and AFP-producing cells to proliferating cells occurs, about $50 \%$ of liver cells are engaged in production of albumin and AFP during liver development. This means that embryonic liver consists of about $50 \%$ hepatoblasts, and the other $50 \%$ is composed of endothelial and mesenchymal cells.

It has been proposed that the young mammalian liver is composed of a cell mosaic consisting of functionally heterogeneous hepatocytes, some producing AFP, others albumin (Valet et al., 1981); (Kuhlmann, 1979); (Guillouzo et al., 1979). In this study, we show using a double in situ hybridization that albumin and AFP genes could be co-expressed earlier by hepatoblasts, at the developmental stage when the liver is formed 
(E12) up to new born rats. Previous immunocytochemical analysis in rat and pigs demonstrated that at foetal stage, albumin and AFP genes could be co-expressed by hepatocytes (Carlsson and Ingvarsson, 1979); (Dempo et al., 1983); (Nayak and Mital, 1977). Rat foetuses from seventeen to nineteen days of gestation and newborn animals displayed a simultaneous expression of albumin and alpha-fetoprotein genes by all hepatocytes. However after the first weeks of postnatal life albumin and alpha-fetoprotein genes are expressed respectively by periportal and perivenous hepatocytes (Poliard et al., 1986).

\subsection{Albumin and AFP mRNA expression in developing liver}

The results presented in section 3.5 indicate that albumin and AFP mRNA levels do not develop strictly in parallel with developing liver. In whole liver (in vivo), albumin mRNA remains constant during embryonic stages (between E12 and E16), in contrast, AFP mRNA decreases at 14 days of gestation and maintains until the end of embryonic stage. The highest expression level of both genes was achieved during the foetal stage (E18). Before birth and during the postnatal life, albumin and AFP mRNA expressions are inversely regulated.

Albumin and AFP mRNA levels were measured in explanted hepatoblasts (in vitro) at different developmental stage in view to support the data obtained in whole liver (in vivo). We observed an increase in albumin and AFP mRNA during 16 days of gestation. Albumin mRNA reaches a peak at 18 days of gestation followed by a slight decrease at 20 days of gestation, and then increase at birth and during the postnatal life. However AFP mRNA reaches a peak at 16 days of gestation and then decrease during the following stages. These results are in agreement with previous 
studies performed with northern blot and densitometric analysis of albumin and AFP gene transcripts (Muglia and Locker, 1984). Other studies indicated that from 17 days of gestation up to fifty days after birth, the ratios of AFP synthesis to albumin synthesis and the ratios of AFP mRNA to albumin mRNA decrease gradually (Liao et al., 1980).

\subsection{Synthesis and secretion of albumin and AFP during liver development}

Previously, synthesis of albumin and AFP was investigated during liver development by immunological techniques (Dempo et al., 1983), and the secretion was examined by direct measurement of serum concentration in yolk sac (Gitlin, 1973). Serum concentration of AFP in foetal liver and yolk sac underwent a decrease followed with a rapid fall after birth to reach a low level in adult state. In contrast, albumin concentration increases from low level early in foetal development to high level after birth and throughout adult life (Abelev, 1974); (Ruoslahti and Terry, 1976). Radioactive biosynthetic labelling method has provided a possibility to assess simultaneously synthesis and release of secreting proteins in viable hepatoblasts. During embryonic stage (E12 up to E16), albumin and AFP proteins were maintained in intracellular and extracellular sites of hepatoblasts. At 18 days of gestation we observed an increase of synthesis and secretion of albumin and AFP, followed by a slight decrease at 20 days of gestation. At birth and during postnatal life albumin and AFP genes were inversely regulated. This finding was supported by measurement of the secretion velocity of albumin and AFP in hepatoblasts at developmental stages E12, E14, E16, E18, and adult hepatocytes using pulse chase experiment. The time needed for synthesis and secretion of albumin and AFP was estimated after a chase period of $15,30,45,60,90$, 
120 and 240 min. Previously, it has been examined the residence kinetics for albumin in hepatoma cell line HepG2 using a pulse chase labelling experiment combined with subcellular fractionation. It has been indicated a rapid decrease of albumin in endoplasmatic reticulum and golgi apparatus during a chase period of $40 \mathrm{~min}$ (Bostrom et al., 1986). Hepatoblasts generated from embryonic liver at 12 days of gestation needs $45 \mathrm{~min}$ to release AFP, in contrast at 14,16 and 18 days of gestation AFP was secreted rapidly and at the same time (15 min). The secretion velocity of albumin differed from that of AFP. At 14 and 16 days of gestation albumin was secreted after a chase period of $30 \mathrm{~min}$. However, at 18 days of gestation and at adult state a short time (15 min) for albumin secretion was needed. We thought that at embryonic stage, albumin and AFP need different time for synthesis and secretion. This results are in agreement with the reports by other researchers indicating that different secretory proteins leave endoplasmic reticulum (ER) with different kinetics (Lodish et al., 1983); (Fries et al., 1984). We noticed that hepatoblasts from late developmental stage (E18) suggest a secretion velocity, which is comparable to mature hepatocyte. This might be due to the differentiation process affecting the organelles involved in intracellular transfer and secretion during embryonic development. Hepatoblasts derived from 12 days of gestation displayed a high translation activity after 9 days in culture and secreted albumin continuously even after chase period of $8 \mathrm{~h}$. This observation is comparable to mature hepatocyte and hepatoblasts from late gestation stage, which continuously synthesize and secrete albumin and AFP. 


\subsection{Characterization of intrahepatic bile duct cells during liver development}

Porspero-related homeobox 1 (Prox1), a divergent transcription factor is involved in the liver development. Prox 1 is expressed in the endoderm that gives rise to the liver and pancreatic anlagen (Burke and Oliver, 2002). It is not restricted to early embryonic stages, but persists into adulthood (Dudas et al., 2004). In this study we demonstrated the spatial and temporal distribution of Prox1, cytokeratin-7 and -19 in embryonic and foetal rat liver. It was claimed that the hepatoblasts give rise to definitive hepatocytes and cholangiocytes (Lemaigre and Zaret, 2004). The exact mechanisms through which bipotential hepatoblasts decide to become hepatocytes or biliary epithelial cells are still unclear, although several factors that contribute to this cell fate decision have already been identified (e. g. Notch signalling, (Tanimizu and Miyajima, 2004); (Lemaigre and Zaret, 2004). Intrahepatic bile ducts starts to differentiate at E15.5 in the rat liver. CK-19 is detected early in the cells that are committed to the bile duct epithelial lineage, while CK-7 is expressed in the biliary epithelial cells in the late gestational stage (Shiojiri, 1997), (Kiiasov et al., 1997).

In the rat embryonic liver (at E12 and E14), Prox1 and CK-19 were found to be expressed in the same cells (hepatoblasts), but these two markers have shown trends of separated expression at early and late developmental stage. Double-positive cells (CK-19/Prox1) were found in the foetal stage (E18) and CK-7 was found only in Prox1 negative cells. In cultured hepatoblasts derived from different developmental stages, immunoreactions with Prox1 and CK-19 antigens has revealed that foetal liver (E18) contains three cell populations, prox1 positive cells, CK-19 
positive cells and prox1/CK-19 positive cells. This data clearly demonstrates that Prox1 expression is lost from cells committed to the bile duct epithelial fate, and remains expressed in hepatocytes. The fact that Prox1 is not expressed in bile duct epithelial cells, which are probably derived from Prox1-positive hepatoblasts, suggests a specific function of the transcription factor for the hepatic lineage. In the adult rat liver, Prox1 was expressed in the hepatocytes, proved by additional markers as HepPar-1 and connexin 32 (Haruna et al., 1996), (Paku et al., 2004), while CK-19 and CK-7 were expressed in the biliary cells. Double expression patterns were not observed.

\subsection{Effect of interleukin-6 (IL-6) on albumin and AFP gene expression in developing liver}

The results presented in section 3.8 indicated that interleukin-6 (IL-6) has a positive effect on hepatoblasts derived from 14 and 18 days of gestation. After stimulation with IL-6, we observed a decrease in albumin and AFP synthesis and secretion. However in hepatoblasts generated from 12 days of gestation a slight effect on synthesis and secretion of AFP and a high effect on synthesis of albumin were observed. According to this observation, unstimulated hepatoblasts from E12, 14 and 18 days of gestation display differential expression of interleukin-6 receptor (IL-6 rec). Hepatoblasts from E14 and E18 express about $10^{5}$ fold interleukin-6 receptor (IL-6 rec) than hepatoblasts from E12.

Interleukin-6 (IL-6) is known as factor that affects a changing in synthesis of several plasma proteins such as haptoglobin, alpha-fetoprotein, beta 2microglobulin, and fibronectin in the liver during acute phase reaction (Ramadori and Christ, 1999), (Roncero et al., 1995). It has been 
demonstrated that IL-6 causes proliferation and differentiation of rat hepatoblasts derived from 14 days of gestation. On the presence of IL6, albumin producing cells decreased after 8 days in culture. When hepatoblasts were stimulated with IL-6 for 3 days and grown for 5 days without IL-6, a high percentage of albumin producing cells were observed. Besides, albumin and AFP synthesis were suppressed after 8 days and 5 days in culture respectively (Zvibel et al., 2004). The role of IL-6 in liver regeneration is well documented. Mice that were $\mathrm{IL}-6^{-/-}$, as well as knockout mice for Tumour Necrosis Factors- receptor 1 (TNF-R1), had impaired liver regeneration after partial hepatectomy. Administration of exogenous IL-6 to IL-6-deficient mice restored hepatocytes proliferation (Cressman et al., 1996). Oncostatin M (OSM), an interleukin-6 family cytokine, in combination with glucocorticoid, induce maturation of hepatocytes, a paracrine mechanism of hepatogenesis; blood cells, transiently expanding in the foetal liver, produce OSM to promote development of hepatocytes in vivo (Miyajima et al., 2000).

\subsection{Identification of hepatic phenotype in passaged hepatoblasts}

Maintenance of hepatic phenotype for long time in cultured hepatoblasts remains a challenge. Various studies have examined foetal hepatic as well as hepatic stem/progenitor cells and kept them for long-term in culture. However most of these studies were interested in the differentiation process stimulated by different growth factors (Suzuki et al., 2003); (Qin et al., 2004); (Kamiya et al., 2006). Human foetal hepatocytes were kept in primary culture for 2 to 4 months without apparent loss of hepatocytic traits. They displayed high proliferation and the culture can be maintained in serum- free medium in the presence of epidermal growth factor and can be passaged at least twice (Lazaro et al., 2003). It has been demonstrated 
that long-term culture of murine hepatoblasts on laminin produces multipotential hepatic progenitors, which possess a strong proliferative capability, differentiate into both hepatocytes and cholangiocytes, and potentially give rise to pancreatic cells (Tanimizu et al., 2004).

In this study, rat hepatoblasts derived from 12 and 14 days of gestation were passaged three times and mRNA expression as well as synthesis and secretion of the main hepatic markers (albumin and AFP) were examined at each passage using the sensitive methods (real time PCR and radioactive biosynthetic labelling). We observed that hepatoblasts could maintain a hepatic phenotype after one passage. During the second passage the hepatoblasts lost their hepatic phenotype.

\subsection{Regulation of hematopoiesis during liver development}

In this study we examined mRNA-expression of genes coding for factors regulating hematopoiesis such as granulocyte-macrophage colonystimulating factor (GM-CSF), granulocyte colony-stimulating factor (G$\mathrm{CSF}$ ), stem cell factor (SCF) and Erythropoietin (Epo) at different embryonic developmental stage in rat liver. A high expression of genes coding for factors which stimulate erythropoiesis was observed at 12 and 14 days of gestation. These factors were down-regulated during the following developmental stages. The developmental stages E12 and E14 were designated as the embryonic stage at which the primitive (at E12) and definitive (at E14) erythropoiesis occur. It was previously reported that erythropoietin promotes the proliferation and differentiation of erythroid progenitor cells and regulates the number of erythrocytes in peripheral blood (Yoshimura and Arai, 1996; Yoshimura and Misawa, 1998). It acts late in the course of erythropoiesis in combination with other factors such as stem cell factor (SCF), interleukin (IL)-3, IL-4, and granulocyte macrophage colony-stimulating factor (GM-CSF) (Yoshimura and Misawa, 
1998). The proliferation and differentiation of primitive and definitive erythroid cells could be stimulated by GM-CSF independently of erythropoietin receptor (EPOR) and the activity is comparable to that of erythropoietin in definitive, but not primitive erythropoiesis (Hisakawa et al., 2001). In fact, during liver development the change from primitive hematopoiesis into definitive hematopoiesis coincide with a high expression of genes coding for factors stimulating erythropoiesis.

\subsection{The outlook}

The use of radioactive biosynthetic labeling followed by specific immunoprecipitation and SDS-PAGE analysis allowed us to monitor, for first time, the synthesis and secretion of albumin and alpha-fetoprotein in endodermal cells derived from ventral foregut region at 10 days of gestation. We thought that 10 days of gestation (E10) is the earliest time point for the emergence of hepatoblasts. It seems that at the time of initiation of hepatic specification (E10) the complete program already operates, controlling the regulation of gene expression of albumin and AFP. This study provides a basis for further investigations on endodermal cells derived from ventral foregut and their application in the regenerative medicine.

Our cellular analysis showed the number of albumin and alpha-fetoprotein producing cells develop in a similar way up to E18. During this time there is an increase of the ratio of albumin and alpha-fetoprotein producing cells to proliferating cells as the liver develops and increases in size. Despite this, we found the ratio of albumin and alpha-fetoprotein to the total number of liver cells remained at 50\% throughout liver development. After 18 days of gestation the ratio of albumin producing cells to proliferating cells continues to increase until adulthood. In contrast, the ratio of alpha- 
fetoprotein producing cells to proliferating cells reaches a maximum and thereafter decreases. The co-expression (co-localisation) of albumin and alpha-fetoprotein is not restricted to the foetal stage. Both plasma proteins were co-expressed by hepatoblasts from 12 days of gestation, the time when the embryonic liver appears, till birth.

Quantitative analysis revealed that at 18 days of gestation, albumin and alpha-fetoprotein mRNA production reaches a maximum and a high level of synthesis and secretion of albumin and alpha-fetoprotin was observed. Additionally, it was found that at the embryonic stage (from E12 up to E16) alpha-fetoprotein was synthesized and secreted at a higher rate than albumin even though the number of albumin and alpha-fetoprotein producing cells is similar. After 18 days of gestation to birth the kinetics for synthesis and secretion of albumin is similar to that in mature hepatocytes.

Three cell populations, Prox1 positive/CK-19 positive cells, Prox1 negative/CK-19 positive cells and Prox1 positive/ CK-19 negative cells we identified. CK -7 is first detected on the 18 day of gestation. It is expressed by Prox1 negative/CK-19 positive cells. Cultured embryonic liver cells could provide a suitable opportunity to study intrahepatic bile ducts development.

Interleukin-6 (IL-6) has a positive effect on hepatoblasts after 14 days of gestation

The hepatoblasts lost their hepatic phenotype during the second passage.

During liver development the change from primitive hematopoiesis (E12) to definitive hematopoiesis (from E14 to adulthood) coincide with a high expression of genes coding for factors regulating erythropoiesis 


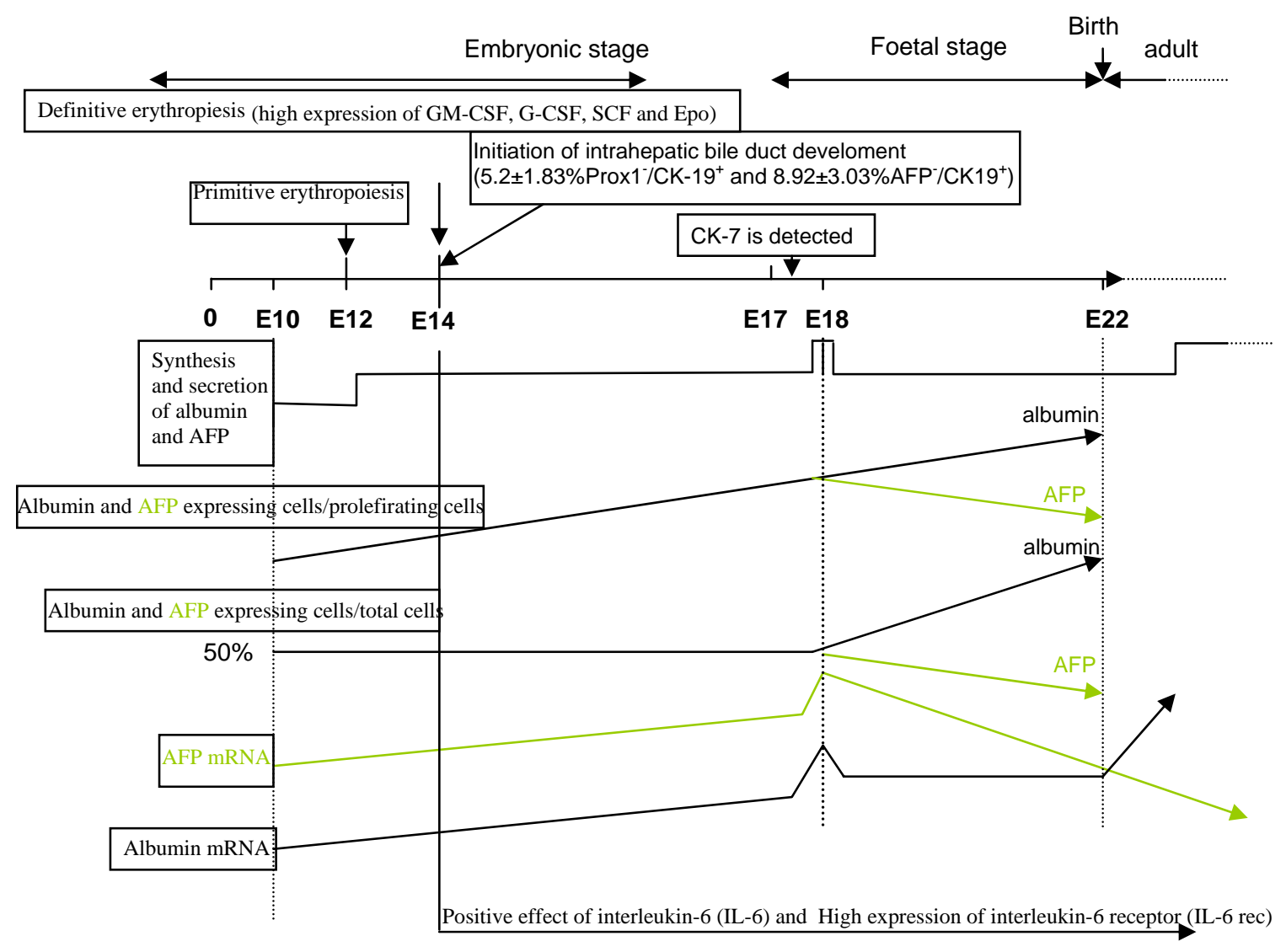

Figure 33: represents the possible changes in characteristics of liver cells during liver development. 


\section{ZUSAMMENFASSUNG}

Die Leber entwickelt sich als Leberknospe aus dem Entoderm des unteren Abschnittes des Vorderdarmes. Die Leberknospe differenziert sich in 2 Teile: In den Leberteil und in den Galleteil. Während der embryonalen Entwicklung differenzieren sich die entodermalen Zellen zu Hepatoblasten, die sich zu reifen Hepatozyten weiter entwickeln können. Ausgehend vom Mesoderm beginnt die Blutbildung im Dottersack. Zum Zeitpunkt der Entstehung hämatopoetischer Knospen in der fetalen Leber beginnt die erste ortständige Blutbildung. Von dort wandern die Stammzellen in die Milz und später in das Osteoid der Knochenanlage, wo sich das Knochenmark entwickelt. Die embryonalen Leberzellen wurden in einigen Studien charakterisiert. Jedoch ihre Charakterisierung während der Leberentwicklung ist bis jetzt noch nicht analysiert worden. Ziel dieser Arbeit war, in einem Rattenmodell, Leberzellen während der Leberentwicklung in vitro und in vivo zu charakterisieren. Wir betrachteten den Fortschritt von der Beginn der Leberentwicklung (E10) bis zum Erwachsenen Leber.

Die embryonale Leber ist durch die Expression von Albumin und Alphafetoprotein (AFP) charakterisiert. In dieser Studie wurden entodermale zellen aus dem Vorderdarmrohr isoliert. Für die Analyse der Synthese und Sekretion von Albumin und AFP in entodermalen Zellen, es wurde die radioaktive biosynthetische Markierung als sehr empfindliche Methode etabliert. Es könnte gezeigt werden, dass Zeigte, dass die Gen-Expresion, Synthese und Sekretion der Albumin und AFP bereits am frühesten Entwicklungsstadium stattfindet. Außerdem, es wurde gezeigt, dass entodermale Zellen, Entoderm und Leber Marker wie Beta-catenin, HNF4alpha, Prox1, BMP-4, Foxa2 und GATA-4 expremieren können. 
Am zweiten Teil dieser Arbeit waren wir an der Entwicklung der Leber interessiert, nachdem sie als Organ identifizierbar war (von E12 zu Adultstadium). Mit Hilfe morphometrische Analyse wurde es demonstriert, dass ungefähr 50\% der gesamten Leberzellen während der embryonalen und fötalen Entwicklung Albumin und Alpha-fetoprotein expremieren können. Zusätzlich wurde es gezeigt, dass während des embryonalen Stadiums das Verhältnis der Albumin- und Alpha-fetoprotein expremierenden Zellen zu den stark vermehrten Zellen sich erhöht. Dieses Verhältnis erreichte sein Maximum am 18 Tag der embryonalen Entwicklung. Die funktionelle Analyse hat gezeigt, dass am 18 Tag der embryonalen Entwicklung Albumin und Alpha-fetoprotein mRNAexpression ein Maximum erreicht hatte und dass eine hohe Rate der Synthese und Ausscheidung von Albumin und Alpha-fetoprotein beobachtet wurde. Zusätzlich konnte gezeigt werden, dass am embryonalen Stadium (von E12 bis zu E16) Albumin und Alphafetoprotein mit unterschiedlicher Rate synthetisiert und abgeschieden wurden. Von 18 Tag bis zur Geburt ist die Kinetik der Synthese und der Ausscheidung von Albumin der den reifen Hepatozyten ähnlich.

Drei Zellpopulation wurden in der embryonalen (E12 und E14) und fötalen Leber (E18) identifiziert. Zwei von denen könnten sich entweder zu Hepatozyten (Prox1 positive Zellen/CK19 negative Zellen) oder zur intrahepatischen Gallengang Epithelzellen (Prox1 negative Zellen/CK19 positive Zellen) entwickeln. Die dritte Zellpopulation (positive Zellen für CK19 und prox1) könnte sich zur beiden Hepatozyten und Gallengang Epithelzellen entwickeln. CK-7 positive Zellen wurden erst am 18 Tag der embryonalen Entwicklung identifiziert.

Die Blutbildung wurde in der embryonalen Leber analysiert. Es könnte gezeigt werden, dass die Gene, die die Blutbildung regulieren, wie GMSCF, G-CSF, SCF und EPO, stark am 12 und 14 Tag der embryonalen Entwicklung (E12 und E14) expremiert sind. 


\section{REFERENCE LIST}

Abelev,G.I.: Alpha-fetoprotein as a marker of embryo-specific differentiations in normal and tumor tissues. Transplant.Rev. 20, 337 (1974).

Alison,M.R., P.Vig, F.Russo, B.W.Bigger, E.Amofah, M.Themis and S.Forbes: Hepatic stem cells: from inside and outside the liver? Cell Prolif. 37, 1-21 (2004).

Ang,S.L., A.Wierda, D.Wong, K.A.Stevens, S.Cascio, J.Rossant and K.S.Zaret: The formation and maintenance of the definitive endoderm lineage in the mouse: involvement of HNF3/forkhead proteins. Development 119, 1301-1315 (1993).

Avital,I., C.Feraresso, T.Aoki, T.Hui, J.Rozga, A.Demetriou and M.Muraca: Bone marrow-derived liver stem cell and mature hepatocyte engraftment in livers undergoing rejection. Surgery 132, 384-390 (2002).

Belanger,L., S.Roy and D.Allard: New albumin gene 3' adjacent to the alpha 1-fetoprotein locus. J.Biol.Chem. 269, 5481-5484 (1994).

Belo,J.A., D.Bachiller, E.Agius, C.Kemp, A.C.Borges, S.Marques, S.Piccolo and E.M.De Robertis: Cerberus-like is a secreted BMP and nodal antagonist not essential for mouse development. Genesis. 26, 265-270 (2000).

Bisgaard,H.C., P.Nagy, P.T.Ton, Z.Hu and S.S.Thorgeirsson: Modulation of keratin 14 and alpha-fetoprotein expression during hepatic oval cell proliferation and liver regeneration. J.Cell Physiol 159, 475-484 (1994). 
Bossard,P. and K.S.Zaret: GATA transcription factors as potentiators of gut endoderm differentiation. Development 125, 4909-4917 (1998).

Bostrom,K., M.Wettesten, J.Boren, G.Bondjers, O.Wiklund and S.O.Olofsson: Pulse-chase studies of the synthesis and intracellular transport of apolipoprotein B-100 in Hep G2 cells. J.Biol.Chem. 261, 13800-13806 (1986).

Burke,Z. and G.Oliver: Prox1 is an early specific marker for the developing liver and pancreas in the mammalian foregut endoderm. Mech.Dev. 118, 147-155 (2002).

Carlsson,R.N. and B.I.Ingvarsson: Localization of alpha-fetoprotein and albumin in pig liver during fetal and neonatal development. Dev.Biol. 73, 1-10 (1979).

Chang,K.T., L.Sefc, O.Psenak, M.Vokurka and E.Necas: Early fetal liver readily repopulates B lymphopoiesis in adult bone marrow. Stem Cells 23, 230-239 (2005).

Chirgwin,J.M., A.E.Przybyla, R.J.MacDonald and W.J.Rutter: Isolation of biologically active ribonucleic acid from sources enriched in ribonuclease. Biochemistry 18, 5294-5299 (1979).

Chui,D.H. and B.V.Loyer: Foetal erythropoiesis in steel mutant mice. II. Haemopoietic stem cells in foetal livers during development. Br.J.Haematol. 29, 553-565 (1975).

Cressman,D.E., L.E.Greenbaum, R.A.DeAngelis, G.Ciliberto, E.E.Furth, V.Poli and R.Taub: Liver failure and defective hepatocyte regeneration in interleukin-6-deficient mice. Science 274, 13791383 (1996).

Dabeva,M.D., P.M.Petkov, J.Sandhu, R.Oren, E.Laconi, E.Hurston and D.A.Shafritz: Proliferation and differentiation of fetal liver epithelial 
progenitor cells after transplantation into adult rat liver.

Am.J.Pathol. 156, 2017-2031 (2000).

Dempo,K., M.Sasaki, T.Kaku, M.Satoh, M.Oyamada and M.Mori:

Immunohistochemical studies on alpha-fetoprotein- and albumincontaining cells in the liver during ontogenesis and early stage of 3'Me-DAB hepatocarcinogenesis. Ann.N.Y.Acad.Sci. 417, 195-202 (1983).

Desmet V. J.: Organizational principles. In The liver - Biology and pathobilogy, ed. Arias I. M. Raven Press NY, pp. 3-14 (1994)

Deutsch,G., J.Jung, M.Zheng, J.Lora and K.S.Zaret: A bipotential precursor population for pancreas and liver within the embryonic endoderm. Development 128, 871-881 (2001).

Dudas,J., M.Papoutsi, M.Hecht, A.Elmaouhoub, B.Saile, B.Christ, S.I.Tomarev, C.S.von Kaisenberg, L.Schweigerer, G.Ramadori and J.Wilting: The homeobox transcription factor Prox1 is highly conserved in embryonic hepatoblasts and in adult and transformed hepatocytes, but is absent from bile duct epithelium. Anat.Embryol.(Berl) 208, 359-366 (2004).

Duncan,S.A., K.Manova, W.S.Chen, P.Hoodless, D.C.Weinstein, R.F.Bachvarova and J.E.Darnell, Jr.: Expression of transcription factor HNF-4 in the extraembryonic endoderm, gut, and nephrogenic tissue of the developing mouse embryo: HNF-4 is a marker for primary endoderm in the implanting blastocyst. Proc.Natl.Acad.Sci.U.S.A 91, 7598-7602 (1994).

Fries,E., L.Gustafsson and P.A.Peterson: Four secretory proteins synthesized by hepatocytes are transported from endoplasmic reticulum to Golgi complex at different rates. EMBO J. 3, 147-152 (1984). 
Fukuda,T.: Fetal hemopoiesis. II. Electron microscopic studies on human hepatic hemopoiesis. Virchows Arch.B Cell Pathol. 16, 249-270 (1974).

Gall,J.A. and P.S.Bhathal: Morphological and immunohistochemical assessment of intrahepatic bile duct development in the rat. J.Gastroenterol. Hepatol. 4, 241-250 (1989).

Gitlin,D.: Ontogeny of fetal plasma proteins. Tumor Res. 8, 1-6 (1973).

Greengard,O., M.Federman and W.E.Knox: Cytomorphometry of developing rat liver and its application to enzymic differentiation. J.Cell Biol. 52, 261-272 (1972).

Grompe, M., M. J. Finegold: Liver stem cells. In Stem Cell Biology, ed. Marshak, D. R., R. L. Gardner and D. Gottlieb, Cold Spring Harbor Laboratory Press, Cold Spring Harbor, NY, pp. 455-497 (2001)

Gualdi,R., P.Bossard, M.Zheng, Y.Hamada, J.R.Coleman and K.S.Zaret: Hepatic specification of the gut endoderm in vitro: cell signaling and transcriptional control. Genes Dev. 10, 1670-1682 (1996).

Guillouzo,A., M.Boisnard-Rissel, L.Belanger and M.Bourel: alpha 1Fetoprotein production during the hepatocyte growth cycle of developing rat liver. Biochem.Biophys.Res.Commun. 91, 327-331 (1979).

Harland,R.M.: In situ hybridization: an improved whole-mount method for Xenopus embryos. Methods Cell Biol. 36, 685-695 (1991).

Haruna,Y., K.Saito, S.Spaulding, M.A.Nalesnik and M.A.Gerber: Identification of bipotential progenitor cells in human liver development. Hepatology 23, 476-481 (1996). 
He,Z.P., W.Q.Tan, Y.F.Tang, H.J.Zhang and M.F.Feng: Activation, isolation, identification and in vitro proliferation of oval cells from adult rat livers. Cell Prolif. 37, 177-187 (2004).

Hebel, R., W. Melvin,: Embryology. In Anatomy and Embryology of the laboratory rat, ed. Stromberg, Biomed Verlag, Germany pp. 231253.(1986)

Hisakawa,H., D.Sugiyama, I.Nishijima, M.J.Xu, H.Wu, K.Nakao, S.Watanabe, M.Katsuki, S.Asano, K.Arai, T.Nakahata and K.Tsuji: Human granulocyte-macrophage colony-stimulating factor (hGMCSF) stimulates primitive and definitive erythropoiesis in mouse embryos expressing hGM-CSF receptors but not erythropoietin receptors. Blood 98, 3618-3625 (2001).

Hong,S.H., E.J.Gang, J.A.Jeong, C.Ahn, S.H.Hwang, I.H.Yang, H.K.Park, H.Han and H.Kim: In vitro differentiation of human umbilical cord blood-derived mesenchymal stem cells into hepatocyte-like cells. Biochem.Biophys.Res.Commun. 330, 1153-1161 (2005).

Jagodzinski,L.L., T.D.Sargent, M.Yang, C.Glackin and J.Bonner: Sequence homology between RNAs encoding rat alpha-fetoprotein and rat serum albumin. Proc.Natl.Acad.Sci.U.S.A 78, 3521-3525 (1981).

Johnson,G.R. and D.C.Barker: Erythroid progenitor cells and stimulating factors during murine embryonic and fetal development. Exp.Hematol. 13, 200-208 (1985).

Jung,J., M.Zheng, M.Goldfarb and K.S.Zaret: Initiation of mammalian liver development from endoderm by fibroblast growth factors. Science 284, 1998-2003 (1999).

Jungermann,K. and R.G.Thurman: Hepatocyte heterogeneity in the metabolism of carbohydrates. Enzyme 46, 33-58 (1992). 
Junqueira,L. C. and J. Carneiro: Histologie, 4. Auflage. ed. T. H. Schiebler Springer-Verlag, Berlin Heiderlberg, Germany (1996)

Kaiser,S: Cell volume regulates liver phosphoenolpyruvate carboxykinase and fructose-1,6-bisphosphatase genes. Am.J.Physiol 274, G509G517(1998).

Kamiya,A., F.J.Gonzalez and H.Nakauchi: Identification and differentiation of hepatic stem cells during liver development. Front Biosci. 11, 1302-1310 (2006).

Kang,X.Q., W.J.Zang, T.S.Song, X.L.Xu, X.J.Yu, D.L.Li, K.W.Meng, S.L.Wu and Z.Y.Zhao: Rat bone marrow mesenchymal stem cells differentiate into hepatocytes in vitro. World J.Gastroenterol. 11, 3479-3484 (2005).

Kania,G., P.Blyszczuk, J.Czyz, A.Navarrete-Santos and A.M.Wobus: Differentiation of mouse embryonic stem cells into pancreatic and hepatic cells. Methods Enzymol. 365, 287-303 (2003).

Kiiasov,A.P., A.A.Gumerova and M.M.Bilalov: [Cytokeratin expression in the pre- and postnatal ontogeny of the rat liver]. Ontogenez 28, 389-393 (1997).

Kubota,H. and L.M.Reid: Clonogenic hepatoblasts, common precursors for hepatocytic and biliary lineages, are lacking classical major histocompatibility complex class I antigen. Proc.Natl.Acad.Sci.U.S.A 97, 12132-12137 (2000).

Kuhlmann,W.D.: Immuno-electron microscopy of alpha 1-fetoprotein during normal development of rat hepatocytes. J.Ultrastruct.Res. 68, 109-117 (1979).

Laemmli,U.K.: Cleavage of structural proteins during the assembly of the head of bacteriophage T4. Nature 227, 680-685 (1970). 
Lazarevich,N.L.: Molecular mechanisms of alpha-fetoprotein gene expression. Biochemistry (Mosc.) 65, 117-133 (2000).

Lazaro,C.A., E.J.Croager, C.Mitchell, J.S.Campbell, C.Yu, J.Foraker, J.A.Rhim, G.C.Yeoh and N.Fausto: Establishment, characterization, and long-term maintenance of cultures of human fetal hepatocytes. Hepatology 38, 1095-1106 (2003).

Lazaro,C.A., J.A.Rhim, Y.Yamada and N.Fausto: Generation of hepatocytes from oval cell precursors in culture. Cancer Res. 58, 5514-5522 (1998).

Lee,C.S., J.R.Friedman, J.T.Fulmer and K.H.Kaestner: The initiation of liver development is dependent on Foxa transcription factors. Nature 435, 944-947 (2005).

Lemaigre,F. and K.S.Zaret: Liver development update: new embryo models, cell lineage control, and morphogenesis. Curr.Opin.Genet.Dev. 14, 582-590 (2004).

Lemire,J.M. and N.Fausto: Multiple alpha-fetoprotein RNAs in adult rat liver: cell type-specific expression and differential regulation. Cancer Res. 51, 4656-4664 (1991).

Liao,W.S., A.R.Conn and J.M.Taylor: Changes in rat alpha 1-fetoprotein and albumin mRNA levels during fetal and neonatal development. J.Biol.Chem. 255, 10036-10039 (1980).

Lichenstein,H.S., D.E.Lyons, M.M.Wurfel, D.A.Johnson, M.D.McGinley, J.C.Leidli, D.B.Trollinger, J.P.Mayer, S.D.Wright and M.M.Zukowski: Afamin is a new member of the albumin, alphafetoprotein, and vitamin D-binding protein gene family. J.Biol.Chem. 269, 18149-18154 (1994). 
Lin,C.S., S.K.Lim, V.D'Agati and F.Costantini: Differential effects of an erythropoietin receptor gene disruption on primitive and definitive erythropoiesis. Genes Dev. 10, 154-164 (1996)

Lodish,H.F., N.Kong, M.Snider and G.J.Strous: Hepatoma secretory proteins migrate from rough endoplasmic reticulum to Golgi at characteristic rates. Nature 304, 80-83 (1983).

Mahieu-Caputo,D., J.E.Allain, J.Branger, A.Coulomb, J.P.Delgado, M.Andreoletti, S.Mainot, R.Frydman, P.Leboulch, J.P.Di Santo, F.Capron and A.Weber: Repopulation of athymic mouse liver by cryopreserved early human fetal hepatoblasts. Hum.Gene Ther. 15, 1219-1228 (2004).

Matsusaka,S., A.Toyosaka, K.Nakasho, T.Tsujimura, A.Sugihara, T.Takanashi, K.Uematsu, N.Terada and E.Okamoto: The role of oval cells in rat hepatocyte transplantation. Transplantation 70, 441-446 (2000).

McLeod,J.F. and N.E.Cooke: The vitamin D-binding protein, alphafetoprotein, albumin multigene family: detection of transcripts in multiple tissues. J.Biol.Chem. 264, 21760-21769 (1989).

Micsenyi,A., X.Tan, T.Sneddon, J.H.Luo, G.K.Michalopoulos and S.P.Monga: Beta-catenin is temporally regulated during normal liver development. Gastroenterology 126, 1134-1146 (2004).

Miyajima,A., T.Kinoshita, M.Tanaka, A.Kamiya, Y.Mukouyama and T.Hara: Role of Oncostatin M in hematopoiesis and liver development. Cytokine Growth Factor Rev. 11, 177-183 (2000).

Molkentin,J.D., Q.Lin, S.A.Duncan and E.N.Olson: Requirement of the transcription factor GATA4 for heart tube formation and ventral morphogenesis. Genes Dev. 11, 1061-1072 (1997). 
Monga,S.P., H.K.Monga, X.Tan, K.Mule, P.Pediaditakis and G.K.Michalopoulos: Beta-catenin antisense studies in embryonic liver cultures: role in proliferation, apoptosis, and lineage specification. Gastroenterology 124, 202-216 (2003).

Muglia,L. and J.Locker: Developmental regulation of albumin and alphafetoprotein gene expression in the rat. Nucleic Acids Res. 12, 6751-6762 (1984).

Naito,M., K.Takahashi and S.Nishikawa: Development, differentiation, and maturation of macrophages in the fetal mouse liver. J.Leukoc.Biol. 48, 27-37 (1990).

Narita,N., M.Bielinska and D.B.Wilson: Wild-type endoderm abrogates the ventral developmental defects associated with GATA-4 deficiency in the mouse. Dev.Biol. 189, 270-274 (1997).

Nayak,N.C. and I.Mital: The dynamics of alpha-fetoprotein and albumin synthesis in human and rat liver during normal ontogeny. Am.J.Pathol. 86, 359-374 (1977).

Neubauer,K., T.Knittel, S.Aurisch, P.Fellmer and G.Ramadori: Glial fibrillary acidic protein--a cell type specific marker for Ito cells in vivo and in vitro. J.Hepatol. 24, 719-730 (1996).

Pack,R., R.Heck, H.P.Dienes, F.Oesch and P.Steinberg: Isolation, biochemical characterization, long-term culture, and phenotype modulation of oval cells from carcinogen-fed rats. Exp.Cell Res. 204, 198-209 (1993).

Paku,S., P.Nagy, L.Kopper and S.S.Thorgeirsson: 2-acetylaminofluorene dose-dependent differentiation of rat oval cells into hepatocytes: 
confocal and electron microscopic studies. Hepatology. 39, 13531361 (2004).

Petkov,P.M., J.Zavadil, D.Goetz, T.Chu, R.Carver, C.E.Rogler, E.P.Bottinger, D.A.Shafritz and M.D.Dabeva: Gene expression pattern in hepatic stem/progenitor cells during rat fetal development using complementary DNA microarrays. Hepatology 39, 617-627 (2004).

Petropoulos,C.J., P.Yaswen, M.Panzica and N.Fausto: Cell lineages in liver carcinogenesis: possible clues from studies of the distribution of alpha-fetoprotein RNA sequences in cell populations isolated from normal, regenerating, and preneoplastic rat livers. Cancer Res. 45, 5762-5768 (1985).

Pillarisetty,V.G., G.Miller, A.B.Shah and R.P.DeMatteo: GM-CSF expands dendritic cells and their progenitors in mouse liver. Hepatology 37, 641-652 (2003).

Poliard,A.M., D.Bernuau, I.Tournier, L.G.Legres, D.Schoevaert, G.Feldmann and J.M.Sala-Trepat: Cellular analysis by in situ hybridization and immunoperoxidase of alpha-fetoprotein and albumin gene expression in rat liver during the perinatal period. J.Cell Biol. 103, 777-786 (1986).

Praloran,V.: Structure, biosynthesis and biological roles of monocytemacrophage colony stimulating factor (CSF-1 or M-CSF). Nouv.Rev.Fr.Hematol. 33, 323-333 (1991).

Pringle,N.P., W.P.Yu, M.Howell, J.S.Colvin, D.M.Ornitz and W.D.Richardson: Fgfr3 expression by astrocytes and their precursors: evidence that astrocytes and oligodendrocytes originate in distinct neuroepithelial domains. Development 130, 93-102 (2003). 
Qin,A.L., X.Q.Zhou, H.Yu, Q.Xie, W.Zhang and Q.Guo: [Effects of growth factors and extracellular matrix on proliferation and differentiation of fetal liver progenitor cell in vitro]. Zhonghua Gan Zang.Bing.Za Zhi. 12, 406-409 (2004).

Quinlan,G.J., G.S.Martin and T.W.Evans: Albumin: biochemical properties and therapeutic potential. Hepatology 41, 1211-1219 (2005).

Ramadori,G. and B.Christ: Cytokines and the hepatic acute-phase response. Semin.Liver Dis. 19, 141-155 (1999).

Ramadori,G., K.H.Meyer zum Buschenfelde, P.S.Tobias, J.C.Mathison and R.J.Ulevitch: Biosynthesis of lipopolysaccharide-binding protein in rabbit hepatocytes. Pathobiology 58, 89-94 (1990).

Ramadori,G. and B.Saile: Mesenchymal cells in the liver--one cell type or two? Liver 22, 283-294 (2002).

Ramadori,G., J.D.Sipe, C.A.Dinarello, S.B.Mizel and H.R.Colten: Pretranslational modulation of acute phase hepatic protein synthesis by murine recombinant interleukin 1 (IL-1) and purified human IL-1. J.Exp.Med. 162, 930-942 (1985).

Rogler,L.E.: Selective bipotential differentiation of mouse embryonic hepatoblasts in vitro. Am.J.Pathol. 150, 591-602 (1997).

Roncero,C., I.Fabregat and M.Benito: Regulation of gene expression by interleukin-6 in fetal rat hepatocyte primary cultures: role of epidermal growth factor and dexamethasone. Hepatology 22, 17691775 (1995).

Rossi,J.M., N.R.Dunn, B.L.Hogan and K.S.Zaret: Distinct mesodermal signals, including BMPs from the septum transversum mesenchyme, are required in combination for hepatogenesis from the endoderm. Genes Dev. 15, 1998-2009 (2001). 
Ruoslahti,E. and W.D.Terry: alpha foetoprotein and serum albumin show sequence homology. Nature 260, 804-805 (1976).

Sansone,G., E.Bronzini, I.Minuto and P.Scartezzini: [Fetal hepatic erythropoiesis. An ultrastructural study. Preliminary report (author's transl)]. Pathologica 71, 243-252 (1979).

Sargent,T.D., L.L.Jagodzinski, M.Yang and J.Bonner: Fine structure and evolution of the rat serum albumin gene. Mol.Cell Biol. 1, 871-883 (1981).

Sasaki,H., M.Nio, D.Iwami, N.Funaki, R.Ohi and H.Sasano: Cytokeratin subtypes in biliary atresia: immunohistochemical study. Pathol.Int.51, 511-518 (2001).

Sasaki,K., H.Iwatsuki, M.Suda and C.Itano: Scavenger macrophages and central macrophages of erythroblastic islands in liver hemopoiesis of the fetal and early postnatal mouse: a semithin light- and electron-microscopic study. Acta Anat.(Basel) 147, 75-82 (1993).

Saunders,M.D., H.M.Shulman, C.S.Murakami, T.R.Chauncey, W.I.Bensinger and G.B.McDonald: Bile duct apoptosis and cholestasis resembling acute graft-versus-host disease after autologous hematopoietic cell transplantation. Am.J.Surg.Pathol. 24, 1004-1008 (2000).

Saxena,R. and N.Theise: Canals of Hering: recent insights and current knowledge. Semin.Liver Dis. 24, 43-48 (2004).

Sekhon,S.S., X.Tan, A.Micsenyi, W.C.Bowen and S.P.Monga: Fibroblast growth factor enriches the embryonic liver cultures for hepatic progenitors. Am.J.Pathol. 164, 2229-2240 (2004). 
Serls,A.E., S.Doherty, P.Parvatiyar, J.M.Wells and G.H.Deutsch: Different thresholds of fibroblast growth factors pattern the ventral foregut into liver and lung. Development 132, 35-47 (2005).

Shamay,A., R.Homans, Y.Fuerman, I.Levin, H.Barash, N.Silanikove and S.J.Mabjeesh: Expression of albumin in nonhepatic tissues and its synthesis by the bovine mammary gland. J.Dairy Sci. 88, 569-576 (2005).

Shiojiri,N.: Development and differentiation of bile ducts in the mammalian liver. Microsc.Res.Tech. 39, 328-335 (1997).

Shiojiri,N., J.M.Lemire and N.Fausto: Cell lineages and oval cell progenitors in rat liver development. Cancer Res. 51, 2611-2620 (1991).

Sigal,S.H., S.Brill, L.M.Reid, I.Zvibel, S.Gupta, D.Hixson, R.Faris and P.A.Holst: Characterization and enrichment of fetal rat hepatoblasts by immunoadsorption ("panning") and fluorescence-activated cell sorting. Hepatology 19, 999-1006 (1994).

Sosa-Pineda,B., J.T.Wigle and G.Oliver: Hepatocyte migration during liver development requires Prox1. Nat.Genet. 25, 254-255 (2000).

Stamp,L., H.A.Crosby, S.M.Hawes, A.J.Strain and M.F.Pera: A novel cellsurface marker found on human embryonic hepatoblasts and a subpopulation of hepatic biliary epithelial cells. Stem Cells 23, 103112 (2005).

Suzuki,A., A.Iwama, H.Miyashita, H.Nakauchi and H.Taniguchi: Role for growth factors and extracellular matrix in controlling differentiation of prospectively isolated hepatic stem cells. Development 130, 2513-2524 (2003). 
Suzuki,A., Y.Zheng, R.Kondo, M.Kusakabe, Y.Takada, K.Fukao, H.Nakauchi and H.Taniguchi: Flow-cytometric separation and enrichment of hepatic progenitor cells in the developing mouse liver. Hepatology 32, 1230-1239 (2000).

Takahashi,K., M.Naito and M.Takeya: Development and heterogeneity of macrophages and their related cells through their differentiation pathways. Pathol.Int. 46, 473-485 (1996).

Tanimizu,N. and A.Miyajima: Notch signaling controls hepatoblast differentiation by altering the expression of liver-enriched transcription factors. J.Cell Sci. 117, 3165-3174 (2004).

Tanimizu,N., H.Saito, K.Mostov and A.Miyajima: Long-term culture of hepatic progenitors derived from mouse Dlk+ hepatoblasts. J.Cell Sci. 117, 6425-6434 (2004).

Teramoto,K., K.Asahina, Y.Kumashiro, S.Kakinuma, R.Chinzei, K.Shimizu-Saito, Y.Tanaka, H.Teraoka and S.Arii: Hepatocyte differentiation from embryonic stem cells and umbilical cord blood cells. J.Hepatobiliary.Pancreat.Surg. 12, 196-202 (2005).

Tian,J.M. and U.Schibler: Tissue-specific expression of the gene encoding hepatocyte nuclear factor 1 may involve hepatocyte nuclear factor 4. Genes Dev. 5, 2225-2234 (1991).

Tournier,I., L.Legres, D.Schoevaert, G.Feldmann and D.Bernuau: Cellular analysis of alpha-fetoprotein gene activation during carbon tetrachloride and D-galactosamine-induced acute liver injury in rats. Lab Invest 59, 657-665 (1988).

Tremblay,K.D. and K.S.Zaret: Distinct populations of endoderm cells converge to generate the embryonic liver bud and ventral foregut tissues. Dev.Biol. 280, 87-99 (2005). 
Tworkowski,K.A., S.E.Salghetti and W.P.Tansey: Stable and unstable pools of Myc protein exist in human cells. Oncogene 21, 8515-8520 (2002).

Valet,J.P., N.Marceau and J.Deschenes: Restricted specialization of differentiating hepatocytes in terms of albumin and alphafetoprotein production. Cell Biol.Int.Rep. 5, 307-314 (1981).

Van Eyken,P., R.Sciot, B.Van Damme, C.Wolf-Peeters and V.J.Desmet: Keratin immunohistochemistry in normal human liver. Cytokeratin pattern of hepatocytes, bile ducts and acinar gradient. Virchows Arch.A Pathol.Anat.Histopathol. 412, 63-72 (1987).

Vassy,J., M.Kraemer, M.T.Chalumeau and J.Foucrier: Development of the fetal rat liver: ultrastructural and stereological study of hepatocytes. Cell Differ. 24, 9-24 (1988).

Wan,Y.J. and J.Y.Chou: Expression of the alpha-fetoprotein gene in adult rat liver. Arch.Biochem.Biophys. 270, 267-276 (1989).

Willert,K. and R.Nusse: Beta-catenin: a key mediator of Wnt signaling. Curr.Opin.Genet.Dev. 8, 95-102 (1998).

Wong,P.M., S.W.Chung, J.S.White, S.M.Reicheld, M.Patterson, B.J.Clarke and D.H.Chui: Adult hemoglobins are synthesized in murine fetal hepatic erythropoietic cells. Blood 62, 1280-1288 (1983).

Yoshimura,A. and K.Arai: Physician Education: The Erythropoietin Receptor and Signal Transduction. Oncologist. 1, 337-339 (1996).

Yoshimura,A. and H.Misawa: Physiology and function of the erythropoietin receptor. Curr.Opin.Hematol. 5, 171-176 (1998).

Yoon,B.I., Y.K.Choi and D.Y.Kim: Differentiation processes of oval cells into hepatocytes: proposals based on morphological and 
phenotypical traits in carcinogen-treated hamster liver. J.Comp Pathol. 131, 1-9 (2004).

Zaret,K.S.: Molecular genetics of early liver development.

Annu.Rev.Physiol 58, 231-251 (1996).

Zaret,K.S.: Regulatory phases of early liver development: paradigms of organogenesis. Nat.Rev.Genet. 3, 499-512 (2002).

Zhao,R., A.J.Watt, J.Li, J.Luebke-Wheeler, E.E.Morrisey and S.A.Duncan: GATA6 is essential for embryonic development of the liver but dispensable for early heart formation. Mol.Cell Biol. 25, 2622-2631 (2005).

Zvibel,I., S.Brill, R.Kariv, A.Traister, T.Golan, J.Chebath, Z.Halpern, M.Revel and R.Oren: Chimeric molecule IL-6/soluble IL-6 receptor is a potent mitogen for fetal hepatocytes. J.Cell Physiol. 200, 245252 (2004). 


\section{ACKNOWLEDGMENTS}

I am very grateful for the tremendous scientific direction and environment provided to me by my thesis advisor Prof. Dr. Dr. Med Giuliano Ramadori; director of department of gastroenterology and endocrinology in clinical university of Göttingen. He allows all students to grow as scientists, and as people, in their own unique way. Working under his direction has been an honor and a privilege, one that I am very thankful for.

I would like to thank Prof. Dr Rüdiger Hardeland, for supervising my PhD thesis and fruitful suggestions during preparation of my thesis and to Prof. Ernst Wimmer for being co-referent for my dissertation in the Faculty of Biology at University of Göttingen.

I am certainly appreciative of all my colleagues for their support and friendship. They provided me excellent lab environment during experimental work.

A special thanks to my colleagues and my friends Dr. Jusef Dudas, Dr. Hassan Dihazi, Dr. Silke Cameron and Dr. Robert Cameron for their support and advice during my doctoral work.

A very special thanks to my friends in Germany and in Marokko for their support and encouragement

At the heart of my support system were my parents Hassan and Zahia elmaouhoub, my loving wife Khadija Es-sabir and my brothers and sisters. 


\section{Lebenslauf}

$\begin{array}{ll}\text { Name: } & \text { Elmaouhoub } \\ \text { Vorname } & \text { Abderrahim } \\ \text { 13.03.1968 } & \text { geboren in Rabat (Marokko) } \\ \text { 1975-1988 } & \text { Grundschule und Gymnasium (Abitur) } \\ \text { 1988-1993 } & \text { Biologie Studium an der Universität Mohamed } \\ \text { V (Rabat)Abschluss als Diplom Biologe } & \text { Deutschsprache im Goethe Institut } \\ \text { 1994-1995 } & \text { Deutschsprache an der Universität Carl von } \\ \text { Ossietzky Oldenburg } & \text { Biotechnologie Studium an der technischen } \\ \text { Universität Berlin, Abschluss als Diplom- } \\ \text { Ingenieur Biotechnologe, Diplomarbeit an der } \\ \text { Geos-laufend }\end{array}$

